\title{
ELECTROMAGNETIC STIRRING WITH ALTERNATING CURRENT DURING ELECTROSLAG REMELTING
}

\author{
By \\ Jose Bernardo Hernandez-Morales \\ B. Sc. (Metallurgical Eng.) University of Mexico, Mexico City, 1983
}

A THESIS SUBMITTED IN PARTIAL FULFILLMENT OF

THE REQUIREMENTS FOR THE DEGREE OF

Master of Applied Science

in

THE FACULTY OF GRADUATE STUDIES

METALS AND MATERIALS ENGINEERING

We accept this thesis as conforming

to the required standard

THE UNIVERSITY OF BRITISH COLUMBIA

October 1988

(c) Jose Bernardo Hernandez-Morales, 1988 
In presenting this thesis in partial fulfilment of the requirements for an advanced degree at the University of British Columbia, I agree that the Library shall make it freely available for reference and study. I further agree that permission for extensive copying of this thesis for scholarly purposes may be granted by the head of my department or by his or her representatives. It is understood that copying or publication of this thesis for financial gain shall not be allowed without my written permission.

Department of Metals and Materials Ensineexing

The University of British Columbia Vancouver, Canada

Date Oct.17, 1988 


\begin{abstract}
The electroslag remelting process combines both a chemical refining ability and a better control on solidification which produces materials of more uniform properties. In spite of the advantages of this process, segregation-prone alloys are still difficult to produce via ESR, particularly when large ingot diameters are involved. In this context the study of externally applied electromagnetic fields is of great interest.

An AC operated stirring device was designed and laboratory-scale experiments were conducted. The solidification structures resulting from the stirring as well as the mixing conditions prevailing in the liquid pool were investigated. Also, modelling studies on the electromagnetic body force produced by the stirrer and the resulting thermal field were conducted.

The start of stirring results in a clearly defined band in both steady- and unsteadystate regimes. The microstructure was not significantly modified in the center of the ingot but a loss in directionality was found at the mould wall. It seems apparent that the stirred liquid does not penetrate significantly inside the interdendritic region. Mixing studies revealed that the degree of mixing in the pool is enhanced by the stirring.

The theoretical calculations show that most of the electromagnetic body force is confined to the magnetic skin depth at the mould wall and the metal-slag interface. Using a previously developed two-dimensional heat transfer model it was not possible to reproduce the experimental pool profiles obtained when stirring was applied.
\end{abstract}




\section{Table of Contents}

$\begin{array}{ll}\text { Abstract } & \text { ii }\end{array}$

Table of Contents

List of Tables

$\begin{array}{lc}\text { List of Figures } & \text { vii }\end{array}$

List of Symbols $\quad$ xi

Acknowledgement $\quad$ xiv

1 Introduction $\quad 1$

1.1 Magnetic Fields in Metallurgical Processes . . . . . . . . . . . . 2

1.1.1 Thermal Effect ................... 2

1.1.2 Hydrodynamic Effect . . . . . . . . . . . . . . 3

1.1.2.1 Induction Furnaces ............... 8

1.1.2.2 Hall-Héroult cell . . . . . . . . . . . . . 8

1.1.2.3 Arc Melting and Welding ............ 9

1.1.2.4 Electromagnetic Casting . . . . . . . . . . . 10

1.1.2.5 Levitation Melting .............. 11

1.1.2.6 Electromagnetic Transport of Liquid Metals ...... . 11

1.1.2.7 Electromagnetic Stirring . . . . . . . . . . . 12

1.2 Influence of Fluid Flow on Segregation . . . . . . . . . . . . 13 
1.2 .1 Segregation . . . . . . . . . . . . . 13

1.2.1.1 Microsegregation . . . . . . . . . . . 14

1.2.1.2 Macrosegregation . . . . . . . . . . . 16

1.3 Segregation in ESR . . . . . . . . . . . . . . 22

1.4 Liquid Movement in ESR . . . . . . . . . . . . . . . 24

1.5 Electromagnetic Stirring during ESR . . . . . . . . . . 27

2 Mathematical Simulation $\quad \mathbf{4 0}$

2.1 Thermal Regime . . . . . . . . . . . . . . . 40

2.2 Electromagnetic Body Force . . . . . . . . . . . . . 41

3 Experimental Procedures

3.1 Experimental Setup . . . . . . . . . . . . . . . . 52

3.2 Experimental Programme . . . . . . . . . . . . . . . 54

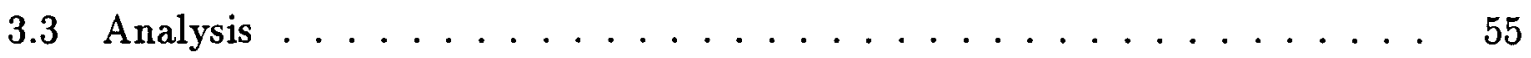

4 Results and Discussion $\quad 67$

4.1 Magnetohydrodynamic Calculations . . . . . . . . . . . 67

4.1 .1 Electromagnetic Field . . . . . . . . . . . . 67

4.1.2 Electromagnetic Body Forces . . . . . . . . . . . . . 69

4.2 Electromagnetic Stirring During ESR . . . . . . . . . . . . 70

4.2 .1 General Observations . . . . . . . . . . . . . . . 70

4.2 .2 Metallurgical Consequences of EMS . . . . . . . . . 72

4.2.2.1 Unsteady-state Stirring . . . . . . . . . . . 72

4.2.2.2 Steady-state Stirring . . . . . . . . . . 74

4.2.2.3 Effect of Pool Profile . . . . . . . . . . . . . 74

4.2.2.4 Liquid Metal Flow . . . . . . . . . . . . 75 
4.3 Mixing in the Liquid Pool ... . . . . . . . . . . . . . 76

4.4 Thermal Regime ....................... 79

5 Summary and recomendations for future work 136

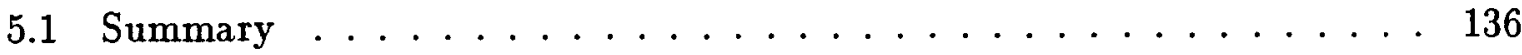

5.2 Recommendations for future work . . . . . . . . . . . 137

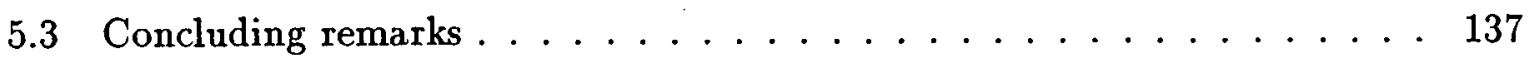

$\begin{array}{lr}\text { Bibliography } & 138\end{array}$ 


\section{List of Tables}

1.1 Mean electromagnetic body force $\ldots \ldots \ldots \ldots$

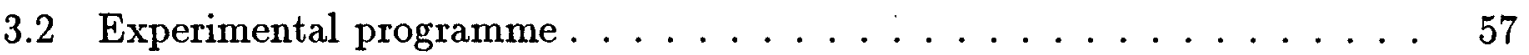

3.3 Chemical composition of slags used in the experimental programme . . 58

3.4 Nominal chemical composition of 4340 alloy steel, in weight percent. . . . 59

3.5 Count acquisition for the standards used in the WDX analysis. . . . . 6 60

4.6 Geometric parameters and electrical properties used in the magnetohydrodinamic calculations. . . . . . . . . . . . . . 82 


\section{List of Figures}

1.1 Schematic illustration of an ESR furnace. . . . . . . . . . . . 31

1.2 Metallurgical applications of EMS. . . . . . . . . . . . . 32

1.3 Interdendritic fluid flow in axisymmetric ingots. . . . . . . . . 33

1.4 Segregation profile and interdendritic fluid velocities in a $\mathrm{Sn}-15 \% \mathrm{~Pb}$ ingot. 34

1.5 Segregation profile and interdendritic flow velocities in a Ni-27\% Mo ingot. 35

1.6 Effect of rotation of mould on flow of interdendritic liquid and macrosegregation in $\mathrm{Sn}-12 \% \mathrm{~Pb}$ ingots. . . . . . . . . . . . 36

1.7 Segregation as function of rotational speed and solidification rate $\ldots 37$

1.8 Flow patterns and macrosegregation. . . . . . . . . . . . 38

1.9 Comparison between experimental and theoretical segregation profiles in a $\mathrm{Pb}-26.5 \% \mathrm{Sn}$ ingot. . . . . . . . . . . . . . 39

2.10 Heat balance over a volume element. (a) Isotropic $k_{\text {eff }}$ (b) anisotropic $k_{\text {eff }} \quad 49$

2.11 Vector potential induced by current flowing through a circular path. . . 50

2.12 Model used to calculate magnetohydrodynamic quantities. . . . . . . . 51

3.13 Magnetic field damping caused by the magnetic skin effect. . . . . . . . 61

3.14 Schematic view of the stirring mould. (a) Top view. (b) Lateral view. . . 62

3.15 Graphite pieces used in the stirring mould. . . . . . . . . . . . 63

3.16 Detail of the stirring mould. . . . . . . . . . . . . . . . . 64

3.17 Mould arrangment used in the experimental programme. . . . . . . 65

3.18 Samples from ingot VII. . . . . . . . . . . . . . . . 66 66 
4.19 Magnetic field distribution obtained for the conditions shown in table 4.6. 83

4.20 Magnetic field distribution obtained for $\omega=20 \mathrm{~Hz}$. . . . . . . . . 84

4.21 Magnetic field distribution obtained for $\omega=1000 \mathrm{~Hz}$. . . . . . . . . . 85

4.22 Magnetic field distribution obtained for an exciting current of 1000 A. . . 86

4.23 Magnetic field distribution obtained for an exciting current of $50 \mathrm{~A}$. . . . 87

4.24 Magnetic field distribution obtained when the coil has only one turn. . . 88

4.25 Magnetic field distribution obtained when the coil has only 2 turns. . . . 89

4.26 Magnetic field distribution obtained for the coil geometry shown. . . . . 90

4.27 Magnetic field distribution obtained for the coil geometry shown. . . . . 91

4.28 Magnetic field distribution obtained for the coil geometry shown. . . . . . 92

4.29 Current density distribution used in the electromagnetic body force calcu-

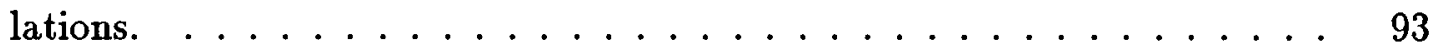

4.30 Electromagnetic body force distribution resulting from the interaction of the fields shown in figs. 4.29 and $4.19 . \ldots . \ldots$. . . . . . . . 94

4.31 Electromagnetic body force distribution for a $500 \mathrm{~mm}$ dia. ingot. Melting current is $10 \mathrm{kA} \ldots \ldots \ldots$. . . . . . . . . . . . . . . . . . . 95

4.32 Detail of the ingot surface showing the effect of slag rotation. . . . . . . 96

4.33 Melting rate as a function of time for ingot XI (stirred) . . . . . . . . . 97

4.34 Energy efficiency as a function of time for ingot XI (stirred). . . . . . . . 98

4.35 Photomacrograph of ingot II . . . . . . . . . . . . . . . . . . 99

4.36 Photomacrograph of ingot III . . . . . . . . . . . . . . . . 100

4.37 Photomacrograph of ingot IV. . . . . . . . . . . . . . . . . 101

4.38 Detail of the stirred zone in ingot III. . . . . . . . . . . . . . 102

4.39 Mushy zone height, temperature gradient and solidification rate for the experimental conditions. Obtained with a two-dimensional, steady-state heat transfer model $[131] . \ldots \ldots$. . . . . . . . . . . . 103 
4.40 Photomicrograph of the stirred zone in ingot II. . . . . . . . . . . . 104

4.41 Analysis of a segregated area in the stirred zone in ingot II. (a) SEM picture. (b) Phosphorus segregation. . . . . . . . . . . . . . 105

4.42 Photomicrograph of the stirred zone in ingot II showing an area perpendicular to the area shown in fig. $4.40 \ldots \ldots \ldots \ldots \ldots$

4.43 Photomacrograph of ingot V . . . . . . . . . . . . 107

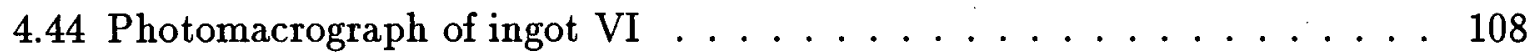

4.45 Photomacrograph of ingot VII. . . . . . . . . . . . . . . 109

4.46 Photomacrograph of ingot VIII. . . . . . . . . . . . . . 110

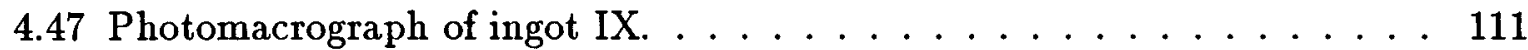

4.48 Photomacrograph of ingot X. . . . . . . . . . . . . . . 112

4.49 Photomicrograph of sample 1 in fig. 3.18 . . . . . . . . . . . . 113

4.50 Photomicrograph of sample 2 in fig. 3.18 . . . . . . . . . . . . . . 114

4.51 Photomicrograph of sample 3 in fig. 3.18 . . . . . . . . . . . 115

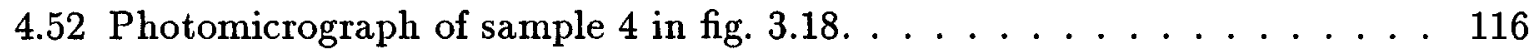

4.53 Photomicrograph of a cross-sectional sample at the ingot surface (non stirred zone). . . . . . . . . . . . . . . . . 117

4.54 Photomicrograph of a cross-sectional sample at the ingot surface (stirred

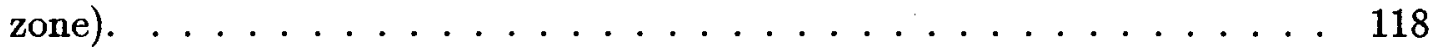

4.55 Nickel and tungsten concentration along the longitudinal axis. Ingot XI. 119

4.56 Nickel and tungsten concentration along the longitudinal axis. Ingot XII. 120

4.57 Effective distribution coefficient for experiments with and without stirring. 121

4.58 Dispersion number for experiment XII (with stirring) . . . . . . . . . 122

4.59 Dispersion number for experiment XI (without stirring) . . . . . . . 123

4.60 Pool profile for a hypothetical 4340 alloy steel ingot. . . . . . . . . . . . 124

4.61 Pool profile for a hypothetical $\mathrm{Cu}-50 \% \mathrm{Fe}$ ingot. . . . . . . . . . 125 
4.62 Pool profile for a hypothetical Inconel 718 ingot. . . . . . . . . . . . 126

4.63 Top temperature distributions used in the study of its effect on the thermal

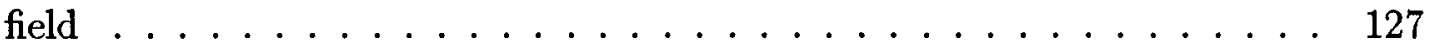

4.64 Pool profile resulting when curve (a) in fig. 4.63 is used. . . . . . . . . 128

4.65 Pool profile resulting when curve (b) in fig. 4.63 is used. . . . . . . . 129

4.66 Pool profile resulting when curve (c) in fig. 4.63 is used. . . . . . . . . 130

4.67 Pool profile resulting when an isotropic $k_{\text {eff }}$ equivalent to $5 \times k_{\text {at }}$ is used. 131

4.68 Pool profile resulting when an isotropic $k_{\text {eff }}$ equivalent to $10 \times k_{\text {at }}$ is used. 132

4.69 Pool profile resulting when an anisotropic $k_{\text {eff }}$ is used. The ratio $k_{\text {eff }}^{z} / k_{\text {eff }}^{\mathrm{r}}$ is

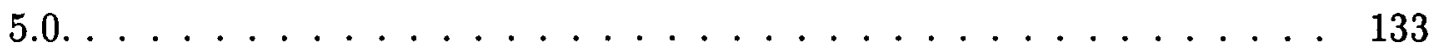

4.70 Pool profile resulting when an anisotropic $k_{\text {eff }}$ is used. The ratio $k_{\text {eff }}^{\mathrm{z}} / k_{\text {eff }}^{\mathrm{r}}$ is

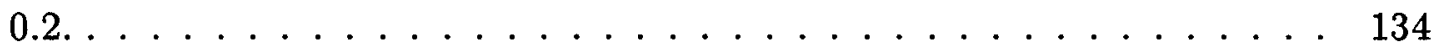

4.71 Same as fig. 4.70 except that $k_{\text {eff }}$ has been increased to $5.0 \ldots \ldots \ldots 135$ 


\section{List of Symbols}

$\vec{A}, \overrightarrow{A_{\theta}}-$ Vector potential and its $\theta$-component, $\mathrm{N} / \mathrm{m}^{2}$

$\vec{B}, \vec{B}_{o}$ - magnetic flux density, $\mathrm{Wb} / \mathrm{m}^{2}$

$C_{L}-$ liquid composition, w/o

$C_{s}-$ solid composition, w/o

$C_{E}-$ eutectic composition, w/o

$\bar{C}-$ average composition, w/o

$D$ - diffusion coefficient, $\mathrm{m}^{2} \mathrm{~s}^{-1}$

$D_{i}$ - ingot diameter, $\mathbf{m}$

$D_{e} \quad$ - electrode diameter, $\mathrm{m}$

$\vec{E}$ - Electric field, V

$f$ - frequency, $\mathrm{Hz}$

$\vec{F} \quad-$ non-conservative part of $\vec{F}_{m}, \mathrm{~N} / \mathrm{m}^{3}$

$\vec{F}_{m} \quad$ instantaneous electromagnetic body force, $\mathrm{N} / \mathrm{m}^{3}$

$\left\langle\vec{F}_{m}\right\rangle$ - mean electromagnetic body force, $\mathrm{N} / \mathrm{m}^{3}$

$g_{l}$ - liquid volume fraction, dimensionless

$g_{E}-$ eutectic volume fraction, dimensionles

$g_{s}$ - solid volume fraction, dimensionles

$\vec{g}$ - acceleration due to gravity, $9.8 \mathrm{~m} / \mathrm{s}^{2}$

$h$ - height of the discrete element, $\mathrm{m}$

$\vec{H}, \vec{H}_{o} \quad$ - magnetic field intensity, A/m

$I, I_{o}$ - electric current, A 


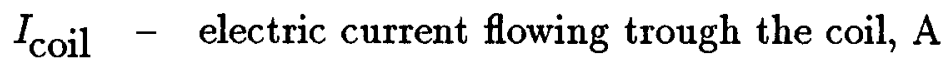

$\jmath-\sqrt{-1}$

$\vec{J}, \vec{J}_{o}-$ current density, $\mathrm{A} / \mathrm{m}^{2}$

$K$ - specific permeability, $\mathrm{cm}^{2}$

$k_{o}-$ equilibrium distribution coefficient, dimensionless

$k_{\text {at }}-$ atomic thermal conductivity, $\mathrm{W} \mathrm{m}^{-1}$

$k_{\text {eff }}^{*}-$ effective distribution coefficient, dimensionless

$k_{\text {eff }}-$ effective thermal conductivity, $\mathrm{W} \mathrm{m}^{-10} \mathrm{C}^{-1}$

$k_{\text {eff }}^{\mathrm{z}}-$ axial effective thermal conductivity, $\mathrm{W} \mathrm{m} \mathrm{m}^{-1} \circ \mathrm{C}^{-1}$

$k_{\text {eff }}^{\mathrm{r}}-$ radial effective thermal conductivity, $\mathrm{W} \mathrm{m} \mathrm{m}^{-1} \circ \mathrm{C}^{-1}$

$L$ - charactheristic length or ingot length, $m$

MR - melting rate, $\mathrm{kg} \mathrm{h}^{-1}$

$\vec{n}$ - unit vector normal to isotherms, dimesnionless

$N_{i}$ - number of nodes,

$P$ - pressure, $\mathrm{N} / \mathrm{m}^{2}$

$r$ - radial direction, $\mathrm{m}$

$R$ - interface ratio of advance, $\mathrm{m} \mathrm{s}^{-1}$

SR - segregation ratio, dimensionless

$R_{m}$ - magnetic Reynolds number, dimensionless

$\vec{S} \quad$ - stirring force density, $\mathrm{N} / \mathrm{m}^{4}$

$T$ - period, $\mathrm{s}$

$t$ - time, $\mathrm{s}$

$\vec{u}$ - isotherm velocity, $\mathrm{m} \mathrm{s}^{-1}$ 


$$
\begin{aligned}
& u_{e} \quad \text { electrode rate of travel, } \mathrm{m} \mathrm{s}^{-1} \\
& \vec{v} \text { - fluid velocity or interdendritic fluid flow } \\
& \text { velocity, } \mathrm{m} / \mathrm{s} \\
& \vec{V} \quad-\text { electrical potential, } \mathrm{V} / \mathrm{m} \\
& z \quad \text { - axial direction or distance from the chill end, } m \\
& \beta-\text { coefficient of thermal expansion, }{ }^{\circ} \mathrm{C}^{-1} \\
& \delta \text { - boundary layer or magnetic skin depth, } \mathrm{m} \\
& \delta_{c} \text { - charge boundary layer, } \mathrm{m} \\
& \gamma \text { - proportionality factor in eq. 1.25, dimensionless } \\
& \eta \quad-\text { magnetic diffusivity, } \mathrm{m}^{2} \mathrm{~s}^{-1} \\
& \mu_{o}-\text { permisivity of free space, } 4 \pi \times 10^{-7} \mathrm{H} / \mathrm{m} \\
& \rho \text { - radius, } \mathbf{m} \\
& \rho_{\mathrm{e}} \quad \text { electrical resistivity, } \Omega \mathrm{m} \\
& \rho_{i} \quad \text { ingot density, } \mathrm{kg} / \mathrm{m}^{3} \\
& \rho_{S} \quad \text { - solid density, } \mathrm{kg} / \mathrm{m}^{3} \\
& \rho_{S E}-\text { solid eutectic density, } \mathrm{kg} / \mathrm{m}^{3} \\
& \rho_{L} \quad-\text { liquid density, } \mathrm{kg} / \mathrm{m}^{3} \\
& \sigma \text { - electrical conductivity, }(\Omega \mathrm{m})^{-1} \\
& \xi \quad \text { - axial distance, } \mathrm{m} \\
& \omega \text { - frequency, } \mathrm{rad} / \mathrm{s} \\
& \phi^{\prime} \quad \text { phase angle, rad } \\
& \Phi \text { - 'electromagnetic pressure', } \mathrm{N} / \mathrm{m}^{3}
\end{aligned}
$$




\section{Acknowledgement}

I would like to thank Dr. Alec Mitchell for his interest and suggestions throughout the course of this work. Several discussions with Dr. F. Weinberg have been also invaluable. The assistance of the technical staff at UBC, in particular, R. Cardeno and M. Mager is greatly appreciated.

Thanks are also extended to my fellow graduate students for their voluntary assistance and to my friends who made this a very enjoyable stay. I would like to express my gratitude to the Universidad Nacional Autónoma de México (Departamento de Ingeniería Química Metalúrgica) and the Department of Metals and Materials Engineering of the University of British Columbia for the financial support given during my professional studies.

Finally, I would like to thank the members of my family for their understanding and encouragment. 


\section{Chapter 1}

\section{Introduction}

The electroslag remelting process (ESR) [1 - 6] is one of the special melting processes which also include vacuum arc remelting, electron beam remelting and plasma arc remelting. The ESR process yields improved ingots by means of both, controlled solidification and chemical refining. It allows a controlled freezing of limited amounts of liquid at a time as opposed to conventional casting. Nevertheless ingot quality deteriorates as ingot diameter and/or melting rate increases [6].

A schematic sketch of an ESR system is shown in fig. 1.1. Alternating or direct current is passed from a solid electrode through molten slag to the base plate which closes the circuit. Since the slag has a high electrical resistivity heat is generated by Joule effect and melts the electrode. The molten metal produced in the form of either droplets or a continuous stream (depending on the melting rate) passes through the slag and builds up a solid ingot. The slag and the ingot are contained inside a water cooled copper crucible. The base plate is also water cooled and it is this heat extraction configuration which produces the highly directional structure characteristic of ESR ingots.

Since most of the alloys fabricated using ESR are segregation prone, factors affecting this phenomenom should be understood in order to increase productivity. The possibility of implementing technologies such as electromagnetic stirring as an aid in improving ingot quality, by controlling segregation and/or modifying the microstructure, should be investigated as well. 


\subsection{Magnetic Fields in Metallurgical Processes}

The thermal and hydrodynamic effects resulting from the interaction between magnetic fields and metals have been used in several metallurgical processes. The most common applications are shown in fig. 1.2. It should be noted that the presence of electromagnetic fields in most of these applications is intrinsic to the process itself, the exception being the use of externally applied fields for stirring.

\subsubsection{Thermal Effect}

Eddy currents can be induced in a bath of liquid metal by a fluctuating magnetic field. These induced currents will then generate heat inside the melt as a result of the Joule effect. This sequence of events constitutes the basis of induction melting where the dissipation of the thermal energy generated in the metal bath is used to melt scrap as well as alloying additions. An induction furnace consists of a coil surrounding a nonconductive (alumina, silica) crucible which contains the metallic charge; a time varying current (with frequency ranging from 50 up to $10000 \mathrm{~Hz}$ ) is passed through the coil generating a fluctuating magnetic field. An important variant of this basic principle is the operation of induction furnaces under vacuum for the production of high temperature steels and superalloys [8 - 10]

The heat generated inside the molten metal can be evaluated from the induced current distribution and the electrical resistivity of the melt. Then a thermal energy balance will give the temperature distribution inside the bath as well as the thermal energy available to melt the scrap and the additions.

Using a dimensional analysis Szekely et al. [12] have shown that the melt is essentially isothermal with temperature gradients present only in the vicinity of the scrap-melt 
boundary. This is a result of the fact that thermal energy is dissipated at a much faster rate than it is generated.

\subsubsection{Hydrodynamic Effect}

The interaction between magnetic fields and current passing through a fluid of very high electrical conductivity (liquid metal or plasma) results in mechanical forces that modify the structure of the flow in that fluid. This coupling between electromagnetic and mechanical phenomena constitutes the basis of magnetohydrodynamics (MHD).

The electromagnetic body force that has to be incorporated in the Navier-Stokes equation is given by

$$
\vec{F}_{m}=\vec{J} \times \vec{B}
$$

Obviously, the first step in order to obtain the velocity field inside the melt or plasma will be to calculate the current density and magnetic field intensity distributions. This task can be done through the solution of the Maxwell's equations [13, pp 10-11] :

$$
\begin{gathered}
\operatorname{div} \vec{B}=0 \\
\operatorname{curl} \vec{E}=-\frac{\partial \vec{B}}{\partial t} \\
\operatorname{curl} \vec{H}=\vec{J} \\
\operatorname{div} \vec{J}=0
\end{gathered}
$$

and the statement of Ohm's law :

$$
\vec{J}=\sigma(\vec{E}+\vec{v} \times \vec{B})
$$


After some algebraic manipulation one can obtain the equation describing the conservation of magnetic field :

$$
\frac{\partial \vec{B}}{\partial t}=\operatorname{curl}(\vec{v} \times \vec{B})+\eta \nabla^{2} \vec{B}
$$

where

$$
\eta=\frac{1}{\mu_{o} \sigma}
$$

is the magnetic diffusivity.

A dimensional analysis of eq. $1.7[14$, p. 17$]$ results in the so-called magnetic Reynolds number defined as $^{1}$ :

$$
R_{m}=\frac{\vec{u} L}{\eta}
$$

By analogy with the ordinary Reynolds number it represents the ratio of convective to diffusive transport of, in this case, magnetic field. In tackling the theoretical analysis of MHD in metallurgical processes this dimensionless number is probably the most important parameter. For most of the cases of interest it can be shown that

$$
R_{m}<<1
$$

which results in diffusion being the dominant mechanism in magnetic field transport and therefore one can expect the current and its magnetic field not to be disturbed by the fluid movement which also means that any electric currents induced by the motion of the fluid across the lines of force will be negligible compared with the electric currents induced by the pulsation of the applied magnetic field [15]. In terms of the solution of the general problem defined by eq. 1.10 it allows the decoupling of eq. 1.7 and Navier-Stokes equation .

\footnotetext{
${ }^{1}$ This is the magnetic Reynolds number based on the velocity as opossed to the one based on the frequency.
} 
It follows that the solution of the MHD applications shown in fig. 1.2 should include the calculation of, in order,

1. Current distribution.

2. Magnetic field distribution.

3. Electromagnetic body force distribution.

4. Velocity distribution.

Since most of the processes involve the presence of thermal gradients which generate buoyancy body forces a thermal balance should also be incorporated.

It should be noted that the electromagnetic contribution to recirculatory flows in liquid metal baths comes from the stirring force density [16]

$$
\vec{S}=\operatorname{curl}(\vec{J} \times \vec{B})
$$

and not from the absolute value of $\vec{F}_{m}$. In other words, the current lines should diverge in order to create vorticity [17].

Moreover, since the frequency used in AC devices is large one will be only interested in the mean value of the Lorenz force given by [18]

$$
<\vec{F}_{m}>\equiv \frac{1}{T} \int_{0}^{T} \vec{J} \times \vec{B} \mathrm{~d} t
$$

While the analytical procedure to find the mean Lorenz force distribution in the melt is quite straigthforward for some idealized cases $[15,16,19]$ numerical solutions are generally required for the solution of practical problems. In this context the drawbacks arising from trying to evaluate eq. 1.12 from magnetic flux measurements only have been pointed out by Meyer et al. [20] and Shercliff [16]. Since the current and magnetic field 
are usually confined to either two-dimensional or axisymmetric conditions Shercliff [16] has proposed the use of the vector potential, $\vec{A}$, as an aid in evaluating $\left\langle\vec{F}_{m}\right\rangle$. This vector is defined by

$$
\vec{B}=\operatorname{curl} \vec{A}
$$

and

$$
\vec{E}=-\frac{\partial \vec{A}}{\partial t}
$$

Alternatively, a comprehensive set of experiments involving measurements of all the parameters involved (current density, magnetic field intensity, phase angle) in the computation of $\vec{F}_{m}$ was performed by Vivés et al. [21] in an induction furnace containing an aluminium alloy. In this experimental programme the current density is evaluated from electric field measurements done with electrical potential sensors while the magnetic field distribution was obtained measuring the voltage across a small coil placed in the liquid and relating it to the r.m.s. value of $\vec{B}$ through the use of Lenz's law. Details of such probes are given in [22]. It should be pointed out that before the advent of this probe there were only velocity measurements available to compare the theoretical results; these measurements were based on either high speed photography of the bath surface $[23,24]$ or drag forces $[25,26]$ (only in low melting point metals).

It is illustrative to examine the result of decomposing the mean Lorentz force into its potential and non-conservative parts $[15]$ :

$$
<\vec{F}_{m}>=\nabla \Phi+\vec{F}
$$

The potential component $(\nabla \Phi)$ in eq. 1.15 can be interpreted as an 'eletromagnetic pressure' which acts on the free surfaces of the melt while the non-conservative part $(\vec{F})$ will result in stirring [11]. 
The importance of obtaining a precise calculation of the electromagnetic body force distribution before engaging in the solution of Navier-Stokes equation has been clearly demonstrated by Lavers [11] who obtained the magnetic force distribution in an induction furnace considering both the presence of a meniscus of a given shape and the common practice of representing the melt by a sharp cornered cylinder.

Although magnetic fields can be produced with either AC or DC, most of the industrial applications use AC fields. A very important effect associated with this kind of field is the magnetic skin effect which results from the interaction between the field and a material of finite electrical resistivity which will act as a sink. The magnitude of this interaction is characterized by the magnetic skin depth, defined as [27]

$$
\delta=\sqrt{\frac{\rho_{e}}{\mu_{o} \pi f}} .
$$

Physically, it represents the distance at which the magnitude of the magnetic field has decayed to $37 \%$ of its original value and is a measure of the ability of a field to penetrate a given material. From eq. 1.16 it can be deduced that a good electrical conductor will absorb a large amount of the field while an insulating material will be almost transparent. Additionally, low frequencies will favour this penetration. Regarding fluid flow generated by magnetic fields, most of the induced turbulence will be confined to a region of the order of the magnetic skin effect. Therefore, classical wall functions used in turbulence models have to be modified accordingly as in, for example, [28].

In the following, MHD phenomena in the metallurgical applications illustrated in fig. 1.2 will be briefly presented. The particular case of the MHD of the ESR process will be discussed in a subsequent section. 


\subsubsection{Induction Furnaces}

As described before (see 1.1.1) an induction coil wound around a crucible is used to create eddy currents inside the metal bath of induction furnaces. Besides the heat generated through the Joule effect the induced currents react with the magnetic field creating a vigorous stirring characterized by a double toroidal pattern. The interest in a detailed study of the flow regime inside the mould is twofold : 1) the stirring is responsible for the very uniform temperature distribution inside the melt and the ability to incorporate alloying additions, and 2) if it is too violent it will erode the refractory walls at a very fast rate causing contamination of the melt and forcing an early replacement of the crucible wall. A very interesting modification to the original design is the use of metallic shields which modify the magnetic field produced by the inductor. These shields are a good example of the flexibilty offered by technology based on MHD.

\subsubsection{Hall-Héroult cell}

The Hall-Héroult cell has been used for the electrolytic production of aluminium since the past century . It consists of an array of carbon anodes submerged in a molten salt that serves as the elecrolyte, electrically connecting the anodes to a bath of molten aluminium (which acts as the cathode) located at the bottom of the cell. Fine particles of alumina are fed into the system and reduced to liquid aluminium and oxygen which in turn reacts with the anodes forming $\mathrm{CO}_{2}$ bubbles. The molten aluminium goes to the bottom of the cell from where it is intermittently extracted. Also, a frozen skin of alumina and electrolyte is formed at the lateral walls of the cell. The passage of current from the carbon anodes through the electrolyte to the molten aluminium bath induces a magnetic field which in turn interacts with the current generating an electromagnetic body force which results in a recirculating pattern. Although the process has been 
used for a number of years, detailed studies of the hydrodynamic phenomena have not been available till recent years. The driving forces behind these types of studies as an aid in cell design include improvement in thermal and electrical efficiencies as well as prevention of short circuits caused by contact between the melt and the anodes resulting from waves at the electrolyte/metal interface. Also, heavy recirculating flow will cause deterioration of the carbon cell lining. In tackling the hydrodynamic description of the cell the complexity of the interrelated phenomena should be realized [29]. The flow in the electrolyte is not only driven by the electromagnetic body force but also by the buoyancy forces created by the $\mathrm{CO}_{2}$ bubbles whose presence makes this a two phase region. In addition, the electrolyte/air and electolyte/aluminium interfaces constitute free boundaries that cannot be approximated as flat surfaces.

\subsubsection{Arc Melting and Welding}

There are several melting and welding processes (arc furnace, vacuum arc remelting, arc welding) which rely on the energy supplied by an arc created between an electrode and the melt surface. Because of the high currents usually employed, a considerable stirring is caused by the interaction of the current with its self-induced magnetic field. It should be pointed out that this interaction not only alters the hydrodynamics of the metal pool but also the structure of the plasma generated within the arc.

In arc furnaces for steelmaking an alternating current in the range of 10 to $100 \mathrm{kA}$ is passed through a graphite electrode generating an arc which is used to melt the metallic charge. A need of a better understanding of such a well established technology is required in order to have a better control on the thermal field and, mainly, the chemical homogenization of the bath. Also, wall erosion produced by excesive stirring should be avoided. 
The vacuum arc remelting process (VAR) is used to produce high quality, segregationfree superalloy ingots [10]. The energy necessary to melt the electrode is provided by a D.C. arc generated between the bottom of the electrode and the top of the remelted ingot, which acts as the anode. Because pool shape and interdendritic fluid velocities are altered by the fluid flow conditions inside the pool it is important to be able to predict the flow behaviour as a function of system geometry and current input. Such an approach would require a good understanding of the hydrodynamic phenomena produced by MHD interactions between the current and the magnetic field that it creates, as well as buoyancy body forces arising from thermal gradients.

In arc welding of metals an arc is created between an inert electrode (cathode) and the metal surface (anode) which will provide the energy required to form a weldpool. The presence of a self-induced magnetic field will create strong stirring in the weldpool which ultimately will affect its quality. It should be pointed out that the fluid flow in the pool is also influenced by thermocapillary forces, i.e., there is a surface tension-driven component usually referred to as Marangoni convection. The familiar counterclockwise pattern produced by the electromagnetic body forces is dominant in most regions of the pool when the surface tension increases with temperature whereas the Marangoni convection plays a more important role in the opposite case.

\subsubsection{Electromagnetic Casting}

The electromagnetic casting (EMC) of round and rectangular ingots of aluminium alloys is currently used to produce more than 450000 ton/year [30]; it has also been applied to copper base alloys [31]. The main advantages of the process are reduction of material losses because of scalping and trimming [32] as well as an improved hot workability [31].

In EMC the role of the conventional mould is now played by radial induced electromagnetic body forces acting on the liquid pool. The many interrelated operational 
parameters involved in the process, e.g., liquid height, geometry, frequency and intensity of the exciting current, superheat, drop rate, water cooling, etc., makes the automatic control of the whole operation very difficult if it is based on semiempirical observations. Although the fluid flow conditions are improved in EMC some other problems are still present i.e, non flatness of the surface and metal overflow. In this context, magnetic screens play an important role in controlling the flow.

\subsubsection{Levitation Melting}

Small amounts of metal (typically in the order of grams) can be levitated and supported against the action of gravity by alternating magnetic fields induced by a high frequency curent passing through a coil. The joule dissipation taking place inside the metal provides enough heat to melt it. Since the need of a crucible is obviated, levitation melting can be very useful in studies of thermodynamics of slags [33] and refining behaviour of refractory metals [34]. It is also a very attractive technique for space processing experiments on solidification, where buoyancy forces are eliminated in abscence of gravity [35].

The equilibrium shape of the melt and the possible existence of convection currents inside it are dependent on the geometric configuration of the coil as well as on the frequency of the exciting current. It is therefore desirable to predict the hydrodynamics of the system in terms of those parameters.

\subsubsection{Electromagnetic Transport of Liquid Metals}

The transport of liquid metal is of great importance regarding automation and energy savings in metallurgical processes. The use of electromagnetic pumps for metal transport in die casting [36,37], as well as in the aluminium industry $[38,39,40,41]$ (where it provides forced convection in melting and holding furnaces, refining and degassing) is of particular interest. A novel application of the same principle is being used in the recovery of 
aluminium scrap [42]. The main feature of these types of pumps is that its operation does not involve any moving parts and the flow can be easily controlled and be continuosly varied using phase shift [43]; the disadvantages are lower discharge velocities [38] and the high cost associated with its ceramic components. Electromagnetic pumps work on the principle of the linear induction motor with the liquid metal acting as the secondary; at the same time that the metal is being transported the channel where it is running is heated by Joule dissipation generated inside the metal. There are two basic types of pumps [43] : submersible and non-submersible. In the case of aluminium alloys three different duct designs have been used.

\subsubsection{Electromagnetic Stirring}

Electromagnetic stirring (EMS) of liquid metals during solidification has become a very important tool in metallurgical processes. Special attention has been devoted to EMS during continuous casting of steel [44], although its application in laddle metallurgy [45], VAR $[46,47]$ and basic solidification studies (see, for example [98]) is well known. The stirring is provided by the Lorenz force generated by the interaction between the induced eddy currents and the magnetic field. In the case of EMS in continuous casting, several inductor arrangments have been proposed; all of them use an exciting current of low frequency to overcome the magnetic shield effect of the copper moulds. Rotating, travelling, and a combination of rotating plus travelling magnetic fields have been used. Although the main advantage of this process is an increased equiaxed zone some other advantages have been claimed. One disadvantage, caused by excesive stirring is the presence of 'white bands' .

In spite of the importance of predicting the flow caused by a given inductor configuration, very few theoretical treatments of the problem are available; the bulk of the 
experimental work being devoted to semiempirical observations of the metallurgical effects.

\subsection{Influence of Fluid Flow on Segregation}

\subsubsection{Segregation}

A phase diagram indicates that during the solidification of an alloy the newly formed solid will have a different composition than the liquid from which it originated. Under ideal circumstances (uniform composition in both liquid and solid phases) one can define the equilibrium distribution coefficient, $k_{o}$, as the ratio of solid to liquid compositions as given by the phase diagram at the temperature of interest [62] :

$$
k_{o}=\frac{C_{S}}{C_{L}}
$$

When the conditions for this equilibrium mode of solidification are met one can expect to have the nominal alloy composition throughout the solid. Unfortunately, in real processes, solidification very seldom occurs under equilibrium and therefore there will always be compositional differences along an ingot or casting. This variation in composition occurs because of redistribution of solute into both solid and liquid phases and it is known as segregation. There are several types of segregation depending on the particular way in which solute is redistributed during solidification. In general, they can be classified as micro and macrosegregation [49]. The former is a variation in solute concentration which extends over small distances (on the order of the primary dendrite arm spacing) while the latter occurs in a larger scale.

Despite the impact that predictive models would have on industrial solidification processes $[50,51,52]$, it has been only until recent years that quantitative descriptions of the solidification phenomena in general, and segregation in particular, have been available. 
The reason for the lack of basic research in this area is the complexity of the solidification process which normally occurs under unsteady-state and non-equilibrium conditions. Its full description requires a good understanding of fluid phenomena in the fully liquid and mushy zones througout the process which in turn depend on the thermal history of both solid and liquid phases. Changes in liquid and solid composition during freezing are interrelated with the thermal field and alter significantly the thermophysical properties of the phases involved. Additionally, the dendritic structure present in most of the industrial processes is geometrically complex.

Flemings [53] has pointed out the importance of mathematical modeling as a tool for understanding and controlling solidification. Due to the complexity of the interrelated phenomena ocurring during solidification, several assumptions had been made during the development of mathematical models describing segregation in ingots and castings which have constrained the applicabilty of such models. From the experimental point of view, the main difficulty arises from the non-transparent nature of liquid metals. In order to overcome this problem, radioactive tracers have been used to follow the different flow patterns during solidification [54,55]. Transparent models using organic compounds have also been employed to get qualitative information on the nature of the solidification process $[56,57,58]$. Recently, in situ electron microscopy studies on solidification of alloys have been reported [59].

\subsubsection{Microsegregation}

Microsegregation in a dendritic array occurs because of lateral solute rejection. Consequently solute concentration varies from the center (where it is a minimum, for alloys with $\left.k_{o}<1\right)$ to the outside surface of a dendrite branch. This phenomenon is usually referred to as coring. In extreme cases a second phase may form between the dendrite 
arms. While this second phase might have a deleterious effect on the mechanical properties of a particular product (as is the case of $\mathrm{M}_{6} \mathrm{C}$ in $\mathrm{M} 2$ tool steel and Laves phases in Inconel 718 [60]) in some cases it is intentionally formed [61].

The degree of microsegregation can be characterized by a parameter known as segregation ratio $[62$, p. 295]

$$
S R=\frac{\text { Max. conc. }}{\text { Min. conc. }}
$$

or the segregation area index [63]. As microsegregation diminishes the segregation ratio tends to 1 while the segregation area index approaches 0 .

Because the time needed for solid-state diffusion is a function of the square of the distance, the extent of residual segregation, i.e., the microsegregation level after postsolidification homogenization, will depend on the dendrite arm spacing.

Correlations between dendrite arm spacing and local solidification time (defined as the time at a given position in an ingot or casting necessary to go from the liquidus to the solidus isotherm) have been reported. A straight line is found when the logarithm of the secondary dendrite arm spacing is plotted against the logarithm of the local solidification time for Al-4.5\% Cu [64] and Pb-Sn [65] alloys. Similar results, expressed in terms of cooling rate, have been reported for cyclohexanol [66], Fe-Ni and Fe-Cr-C [67], and Al-Si, $\mathrm{Al}-\mathrm{Sn}, \mathrm{Al}-\mathrm{Ag}, \mathrm{Sn}-\mathrm{Sb}$ and $\mathrm{Zn}-\mathrm{Sn}[68]$ alloys.

Apart from the dendrite arm spacing, other factors affecting the residual segregation after a homogenization process are the geometry of the isoconcentration surfaces, the diffusion coefficient of the solute and the time of thermal processing [69]. 


\subsubsection{Macrosegregation}

There are several types of the long-range compositional difference known as macrosegregation.

Binary alloys with low solute concentration will solidify with a planar interface. In this case, the first solid formed will have a lower solute concentration (for an alloy of $\left.k_{o}<<1\right)$ than the nominal alloy composition, the solute in excess being rejected at the solidification front and enriching the remaining liquid. This type of segregation is known as normal segregation [70] and would lead to an ingot of low concentration at the surface and high concentration at the centreline with the exact shape of the solute profile being dictated by the mixing conditions in the liquid [62, p. 251]. The two extreme cases are

1. Solute transport in the liquid by diffusion only.

2. Complete mixing in the liquid by diffusion and convection.

In general the intermediate case of partial mixing in the liquid will occur.

A quantitative description of normal segregation can be obtained from a mass balance assuming steady-state which results in

$$
C_{s}=k_{\mathrm{eff}}^{*} C_{o}\left[1-\frac{z}{L}\right]^{k_{\mathrm{eff}}^{*}-1}
$$

where $k_{\text {eff }}^{*}$, the effective distribution coefficient, is defined as

$$
k_{\mathrm{eff}}^{*}=\frac{k_{o}}{k_{o}+\left(1-k_{o}\right) \exp (-R \delta / D)}
$$

When solute transport in the liquid occurs by diffusion only, $k_{\text {eff }}^{*}$ is equal to $k_{o}$ while $1<<k_{\mathrm{eff}}^{*}<<k_{\mathrm{o}}$ for partial mixing.

In this model the solidification process is viewed as composed of a boundary layer in front of the solid-liquid interface where the main solute transport is by diffusion while 
convective transport is assumed in the bulk of the liquid. The greater the degree of mixing the smaller will be the thickness of the boundary layer. Ghias et al. [71] have pointed out the problems arising from the use of this so-called boundary layer model. Besides the fact that this is not a truly boundary layer but a stagnant film model, the assumption of a finite unstirred zone in front of the solid-liquid interface might lead to erroneous conclusions.

The more common case of dendritic growth leads to various forms of macrosegregation [72]. As opposed to what happens during solidification with planar interfaces the segregation pattern of a dendritic array is inverted, i.e., portions of an ingot close to the chill end will be solute-rich while the top of the ingot will be depleted in solute. This behaviour is thus known as inverse segregation [73] . A different type of defect, known as channel-type segregate, is also present. Positive segregation (solute concentration greater than the average) channels with characteristic ' $A$ ' and ' $V$ ' shapes can be found at outer and inner regions, respectively, close to the top of an ingot. A negative cone of segregation will be present near the bottom $[74,75]$.

Chains of equiaxed grains might also be present on the surface of a casting. These zones are enriched in all but the inversely segregated solute and are known as freckles [76]. They are the result of flow of solute-enriched interdendritic liquid due to solidification contraction, gravitational or electromagnetic forces [77] and/or gas evolution [78]. Banding is a kind of macrosegregation that appears as a change in composition and structure in a band parallel to the solid-liquid interface [79]. It results from thermal or mechanical disturbances at the solidification front [80].

Early investigations had related each of the previously described defects to different causes. Flemings et al. [81-83] suggested that this is not the case and proposed a basic mechanism common to all of these defects, namely, interdendritic fluid flow. Based on work by Kirkaldy et al. [84], these researchers developed a local solute redistribution 
equation which takes into account interdendritic solidification shrinkage exclusively.

The main assumptions in that model are :

1. Constant solid density.

2. Negligible solid diffusion.

3. No pore formation.

The general equation derived for the three dimensional case is

$$
\frac{\partial g_{L}}{\partial C_{L}}=-\left(\frac{1-\beta}{1-k_{o}}\right)\left(1+\frac{\vec{n} \cdot \vec{v}}{\vec{n} \cdot \vec{u}}\right) \frac{g_{L}}{C_{L}}
$$

In order to get analytical solutions for some cases of practical interest further assumptions have to be made :

5. The equilibrium distribution coefficient is constant, i.e., the slopes of both liquidus and solidus are constant.

6. The liquid density is constant.

7. The fraction liquid varies linearly with the temperature.

8. Planar isotherms, i.e., unidirectional heat flow.

Once eq. 1.21 is solved the degree of segregation can be calculated from

$$
\bar{C}=\frac{\rho \int_{0}^{1-g_{E}} C_{S} \partial g_{S}+\rho_{S E} g_{E} C_{E}}{\rho_{S}\left(1-g_{E}\right)+\rho_{S E} g_{E}}
$$

and

$$
\Delta C=\bar{C}-C_{o}
$$

Working with radioactive tracers, Hebditch et al. [85] found that gravitationally induced flow in a $\mathrm{Sn}-6 \% \mathrm{Zn}$ occurs in the mushy zone of a casting. Since the densities of 
those elements are very similar, they concluded that even more pronounced effects can be expected in other systems. The gravitational contribution to the interdendritic fluid flow was accounted for by Mehrabian et al. [86] who extended a previous work [81 - 83] including details of the fluid flow in the mushy zone. They obtained the interdendritic fluid velocity by treating the mushy zone as a porous medium which behaves like a bundle of capillary tubes with the dendrite arms acting as the walls. Assuming that Darcy's law [87] applies, the following expresion for the velocity vector was obtained

$$
\vec{v}=-\frac{K}{\mu g_{L}}\left(\nabla P+\rho_{L} \vec{g}\right)
$$

An important parameter in the previous equation is the permeability ${ }^{2}, K$, of the porous medium. In that investigation the authors assumed it to be isotropic and therefore only dependent on the fraction liquid :

$$
K=\gamma g_{L}^{2}
$$

where $\gamma$ depends on geometric factors and was assumed to be constant for a given dendrite arm spacing.

The analytical solution of the equation describing solute redistribution (eq. 1.21) requires some physical approximations which drive the solution from the real problem. The availabilty of numerical techniques has made possible the outcome of more realistic approaches. Ohnaka et al. [89] stressed the shortcoming of having to assume the temperature field across the mushy zone. In order to solve this problem they used the inner nodal point method which allowed them to solve the fluid flow and heat transfer equations alternately. Again, a linear relationship between liquid temperature and fraction solid and between liquid density and temperature in the mushy zone was assumed. Streat et al. [90] studied the macrosegregation occuring in vertically solidified ingots. In that

\footnotetext{
${ }^{2}$ Actually, this is the specific permeability [88].
} 
investigation the interdendritic fluid flow was assumed to be driven by gravitational effects only, neglecting backflow due to solidification contraction. The numerical technique employed permitted the use of variable distribution coefficient and liquid density. The solid-liquid region was, again, approximated by the porous medium model. The calculation of the permeability involved an estimation of the number of channels from measured primary dendrite arm spacings and an experimentally obtained tortuosity factor.

By means of radioactive tracers $\left(\mathrm{Cu}^{64}\right.$ in $\left.\mathrm{Al}-10 \% \mathrm{Cu}\right)$ Prabhakar et al. [91] investigated inverse segregation and found that some solidification variables not included in the previous models can influence the segregation patterns. Based on this observation Minakawa et al. [92] proposed a more comprehensive finite-difference model for predicting inverse segregation. This model includes the change in volume associated with the phase change, density changes in the residual liquid due to changes in concentration, and thermal contraction. Two important parameters, degree of superheat and thermal resistance at the chill end, were also investigated.

Phenomena occuring in the fully liquid zone ahead of the solidification front may alter the interdendritic fluid flow behaviour. Experimental observations [93 - 96] show that the liquid penetration into the mushy zone depends on factors such as temperature and density differences as well as the fluid velocity in the liquid region. Working with brine solutions, Weinberg [93] reported that at high density gradients even forced convection in the liquid cannot effectively alter the fluid in the two phase region because the high density liquid found in the lower part of the pool acts as a barrier which prevents the passage of the less dense liquid. Verhoeven [95] reported observations related to macrosegregation under mixing in the liquid pool ahead of the solidification front. His results for tin alloys show that the temperature gradient is an important factor regarding liquid penetration into the dendritic array. Takahashi [96] found that the washing effect of bulk fluid flow is stopped for a value of solid fraction of 0.67 . 
Zanner et al. [97] developed a mathematical model for describing the heat and fluid flow in vaccum arc remelting furnaces. In this model a different type of boundary condition was defined in the liquid pool, i.e., the immobilization temperature. Physically, this temperature defines the deepest isotherm into the liquid pool at which heat transfer due to liquid movement is still significant, in other words, below this isotherm the liquid mass and velocity are so small that their contribution to the heat transport is negligible. It can also be viewed as a progressive decrease in the liquid motion due to an increase in the drag force caused by the growing solid. From the point of view of the numerical solution of the problem it defines a surface at which the flow can be treated as rigid-body motion. In that particular investigation the immobilization temperature was arbitrarily defined in terms of mass fraction solid, $\left(f_{s}=0.38\right)$.

Many solidification processes have been improved through the use of external devices applied with the aim of enhancing liquid movement during solidification. Although most of these techniques have been studied with the promotion of equiaxed structures or finer grain sizes through mechanical interactions as the main objective, it is expected that they may also alter the interdendritic fluid flow patterns. The influence of externally applied forces on the interdendritic fluid flow was studied by Keane [98]. He implemented a mathematical model and showed that the interaction between applied homogeneous current and an inhomogeneous magnetic field in horizontally solidified ingots can counteract the effect of gravitational forces on interdendritic fluid flow up to the point of supressing macrosegregation. The theoretical predictions were compared with experimental results obtained with $\mathrm{Al}-4.5 \% \mathrm{Cu}$ and a good agreement was found. He also investigated the effect of externally applied centrifugal forces and found similar results. No experimental work was done in this area. 


\subsection{Segregation in ESR}

As discussed before, the flow of interdendritic liquid is mainly responsible for macrosegregation in conventional castings. The same principle applies to ESR $[77,99]$ ingots. Mitchell et al. [99] studied the effect of liquid movement on macrosegregation and heat transfer. They added FeS or $\mathrm{Sn}^{113}$ during the remelting of AISI 1015 and W80 electrodes and concluded that the liquid mixing in the mushy zone will solely dictate the value of the effective distribution coefficient.

A macrosegregation criteria can be defined from the segregation equation presented before (eq. 1.21)

$$
1-\frac{\vec{n} \cdot \vec{v}}{\vec{n} \cdot \vec{u}}=\frac{\rho_{S} g_{L}+g_{E}\left(\rho_{S E}-\rho_{S}\right)}{\rho_{L} g_{L}}
$$

where the dimensionless parameter $-(\vec{n} \cdot \vec{v} / \vec{n} \cdot \vec{u})$ can be interpreted as local flow velocity perpendicular to an isotherm relative to the isotherm velocity. When both terms in eq. 1.26 are equal no macrosegregation should occur. If the right-hand side is smaller more interdendritic fluid will flow from the hotter to the cooler region of the mushy zone resulting in negative segregation. Positive segregation will occur for the opposite case. When the left-hand side of this equation is less than zero some remelting will occur. This type of behaviour is thought to be responsible for the formation of channel-type segregates. Since the liquid density varies throughout the mushy zone several interdendritic fluid flow patterns might develop during solidification under axi-symmetric conditions. This patterns are shown in fig. 1.3.

Following the solute redistribution equation proposed by Flemings and coworkers [81 - 83], Ridder et al. [100] developed a steady-state model which can be used in predicting macrosegregation in ESR ingots. An important feature of this model is that the actual two-dimensional isotherms are used during the calculations. The experimental and theoretical segregation profiles, as well as the calculated interdendritic fluid flow, 
for a $\mathrm{Sn}-15 \% \mathrm{~Pb}$ ingot produced in a simulated ESR apparatus which eliminates the electromagnetic forces present in real systems are shown in fig. 1.4. Similar plots for a $200 \mathrm{~mm}$ diam., Ni-27\% Mo ingot produced by ESR are shown in fig. 1.5. Jeanfils et al. [123] applied the same model to a multicomponent alloy. They found only a qualitative agreement between predicted and measured macrosegregation for a $40 \mathrm{~cm}$ dia. Waspaloy ingot.

Kou et al. [102] studied the effect of rotation on segregation in remelted ingots. They extended the theoretical model proposed by Keane [98] including concave isotherms. Fig. 1.6 shows theoretical results when the mould is rotated at several speeds during remelting. The combined effects of rotational speeds and solidification rates are shown in fig. 1.7. From both figures the influence of the applied force on the flow pattern and hence on the resulting macrosegregation is readily seen. In order to verify the model, experiments were carried out using $\mathrm{Sn}-12.2 \% \mathrm{~Pb}$ alloys. A good agreement between experimental and theoretical segregation patterns was found. The particular alloy used in this study has a liquid density which increases with lead concentration. Additional theoretical calculations are presented for hypothetical alloys with liquid densities decreasing and, increasing and then decreasing with solute concentration, respectively.

Petrakis et al. [103] employed a different method for rotating the system. They rotated the liquid pool ahead of the solidification front. The forced convection induced in the fully liquid zone altered the shape of the mushy region and, at high rotational speeds, even produced a convex liquidus isotherm. They modified the previous mathematical model by changing the boundary condition along the liquidus which now has a different pressure distribution reflecting convection in the liquid. Experimental and theoretical results for two rotational speeds are shown in fig. 1.8. The rotation of the liquid pool results in two opposite effects : the pressure at the center of the ingot is reduced (which drives interdendritic fluid towards the center) while the convex liquid us isotherm causes 
the liquid to flow away from there. The solidification rate is, again, an important overall factor in the final solute distribution.

Ridder et al. [104] included the effect of the motion in the liquid pool due to natural convection only on the segregation pattern. They concluded that very little influence on the interdendritic fluid flow should be expected. Consequently, the predicted segregation will not be considerably affected when natural convection is included in the model as can be seen in fig. 1.9 .

\subsection{Liquid Movement in ESR}

Because of the adverse experimental conditions found in ESR operations, i.e., a high temperature environment and a highly reactive slag, it is difficult to obtain direct measurements regarding the hydrodynamic behaviour of the liquid metal in an ESR operation. Nevertheless, indirect evidence suggests that there is some degree of motion in that zone. Working with a physical model, Campbell [105] observed a very fast diffusion of the superheat accompanying the falling metal droplets which can only be rationalized in terms of convection currents into the metal pool; from the analysis of the macrostructure of quenched samples of laboratory size 18/8 stainless steel ingots Fredricksson et al. [106] suggested that an appreciable movement in the metal pool should be expected. Another indirect evidence of the motion in this region is the fact that several authors have introduced an effective themal conductivity in the metal pool in order to take into account the convective contribution to energy transport. It is important to keep in mind that fluid flow and thermal phenomena in the metal pool are intimately related : liquid movement will contribute to the convective energy transfer while temperature differences will create buoyancy body forces that drive the liquid.

While several studies regarding the movement of the liquid slag have led to a good 
understanding of the main mechanisms responsible for it, the same does not hold for the liquid pool. There are two main reasons for this difference : 1 ) the sources of motion in the liquid pool are different from those found in the slag, and 2) while all the slag boundaries (slag-gas, slag-metal and slag-slag skin) are well defined, the separation between the fully liquid and the mushy zone is not clear.

Four possible sources of movement of the liquid metal in ESR furnaces can be postulated :

1. Momentum carried by the falling droplets.

2. Interactions between slag and metal pool.

3. Buoyancy body force

4. Electromagnetic body force.

Obviously, the first step in characterizing the hydrodynamics in this region is to assess the relative importance of each source. Campbell [105] used a transparent model in which the slag was simulated by the $\mathrm{LiCl}-\mathrm{KCl}$ eutectic and the consumable electrode was made from lead, zinc or aluminium. From his observations on the droplet motion as it enters the liquid pool, he suggested that the momentum accompanying the falling droplets is the main contribution to the movement observed into the liquid pool. An important finding reported in that investigation pointed out the fact that the slag-pool interface behaves as a rigid boundary which results in a complete independency between the two phases from the momentum transfer point of view, i.e., the vigorous motion observed in the liquid slag does not influence the movement in the pool. Samoilovich et al. [107] made a combined fluid flow and heat transfer analysis and reached the same conclusion regarding the importance of the momentum carried by the droplets as they enter the 
liquid pool. Experimental observations by Rawson et al. [108] using a physical model support this probable source of motion.

Mitchell et al. [99] conducted some experimental work with AISI 1015 and W80 steels in a laboratory-scale ESR unit. From attempts to fitting the shape of the experimentally obtained isotherms they postulated that a stronger metal movement in the neighborhood of the metal-mould interface should be present. Since the thermal gradients are steeper at that boundary they concluded that the flow should be driven by thermal convection. Also, in contrast to previous results, they stated that the falling droplets do not have an important contribution to the overall pool mevement. Essentially the same trend of results was reported by Nakamura et al. [109] in a similar experimental program. The results indicate that no stagnant zone should be expected inside the pool. A differential equation for describing the mass transfer assuming complete mixing was proposed and the experimental results also show that fully mixing conditions prevail in the metal pool. Another set of experiments was set up in order to study the coupled effect of slag/metal reactions and mixing in the liquid pool. The experimental values were close to those predicted by a differential equation which incorporates both mixing and chemical reaction effects.

The ESR process is characterized by the passage of a current from an electrode through the liquid slag and metal baths to the base plate. The main purpose of this current is to provide the necessary heat for melting the electrode by means of the Joule effect caused by the high electrical resistivity of the slag. There is another important effect associated with the current flow, namely, the induction of a magnetic field. When this self-induced field interacts with the current passing through the system it produces a mechanical force given by eq. 1.1 :

$$
\vec{F}_{m}=\vec{J} \times \vec{B}
$$


Therefore, the current distribution plays an important role in determining the magnitude of the electromagnetic body force. It is basically defined by the two electrical configurations used in ESR units : 1) live mould, where the current is partially carried by the mould and 2) insulated mould, in which case the current goes completely to the base plate.

There is a number of other factors that can alter the current distribution in an actual operation. Birck et al. [110] found that the depth of electrode immersion modifies the global slag resistance and therefore the loci of isopotential lines. The electrical resistivity of the slag plays an important role in the process and any change on it will be reflected in the actual current path. Obviously, the overall chemical composition of the slag influences its electrical conductivity [111]. Another important factor is the dependence of the electrical resistivity with temperature (this fact being more accentuated in laboratoryscale ESR furnaces where the thermal field is less uniform). Stirring effects due to electromagnetic and buoyancy forces, as well as the presence of metal droplets (which have a lower electrical resistivity) can also affect the overall electrical resistance offered by the slag $[1$, p. 14].

\subsection{Electromagnetic Stirring during ESR}

Among the several techniques proposed to modify the liquid metal movement in processes involving solidification the use of an externally applied electromagnetic field has gained more and more acceptance over the last years. It is important to note that, according to a mathematical model which includes electromagnetic and buoyancy body forces [127], the velocities in the liquid pool of an ESR installation are much more smaller than the ones in the slag and it is, therefore, possible to increase them.

Zabaluev et al. [113] reported a favourable effect of the magnetic field on segregation 
in square ingots ( $415 \times 415 \mathrm{~mm})$ of ShKh15Sgsh and ShKh15Sh steels. Rawson et al. [108] observed a flatter pool profile when a solenoid was surrounding the mould in its physical model. They suggested that the reason for this behaviour is the deflection of the droplets during its falling through the slag (due to their interaction with the magnetic field) which results in a more evenly distributed heat input to the metal pool. Dudko et al. [114] also found a flatter pool profile along with smaller volume and depth of the pool when ingots where cast with A.C. in $100 \mathrm{~mm}$-dia. moulds. They also report an increase of $30 \%$ in the melting rate.

Wagner [115] obtained similar results for Inconel 600 ingots remelted with direct current using a positive electrode. The ingots were $75 \mathrm{~mm}$ in diameter and an increase of $20 \%$ in the melting rate was obtained. The same trend is reported by Nikulin et al. [116] for a D.C. operation coupled with a solenoid fed with direct current. Miyazawa et al. [117] studied the effect of an external magnetic field generated with direct current on the behaviour of a laboratory-scale ESR unit operated in D.C. mode. From the maximum speed of rotation observed at the free surface of the slag they estimated the mean electromagnetic body force and found it lower than that predicted theoretically. They suggested that this difference is entirely due to asymmetry of the applied magnetic field. Since calculations of the current distribution are not presented in detail, it is thought that it could be another source for the observed discrepancy.

Due to the possibilty of operating an ESR machine with either direct or alternating current four different combinations can be set up when using direct or alternating current to feed a solenoid wound around the mould. The resulting mean electromagnetic body force for the possible combinations is shown in table 1.1. 
This force is calculated using eq. 1.12 :

$$
<\vec{F}_{m}>\equiv \frac{1}{T} \int_{0}^{T} \vec{J} \times \vec{B} \mathrm{~d} t
$$

where

$$
\vec{J}=\sqrt{2} \vec{J}_{\circ} \sin (\omega t)
$$

and

$$
\vec{B}=\sqrt{2} \vec{B}_{o} \sin \left(\omega t+\phi^{\prime}\right)
$$

From this expression it can be readily realized that the maximum force (for a given combination of current and magnetic field intensity) will be obtained when the current path intersects the magnetic lines at angles of 90 degrees and the current passing through the system and the one used to feed the coil are on phase. Asai et al. [118] developed an experimental programme covering the conditions presented in table 1.1. Besides obtaining the qualitatively predicted results shown in table 1.1 they also found a deeper pool profile when the external field was applied.

As can be noticed, most of the experimental work on this topic has been carried out using a coil wound around the mould as the source for the magnetic field. Campbell et al. [119] showed that the radial component of the magnetic field can be enhanced by opposing coils placed either side of a compact coil. 
Table 1.1: Electromagnetic body force for the 4 possible combinations of applied current and magnetic field in an ESR furnace.

\begin{tabular}{|c||c|c|}
\hline \multicolumn{1}{|c||}{\multirow{2}{*}{$\begin{array}{c}\text { Magnetic } \\
\text { current } \\
\text { density }\end{array}$}} & \multicolumn{2}{c|}{ Electric current density } \\
\cline { 2 - 3 } & DC & AC \\
\hline \hline DC & $J_{o} B_{o}$ & 0 \\
\hline AC & 0 & $J_{o} B_{o} \cos \left(\phi^{\prime}\right)$ \\
\hline
\end{tabular}




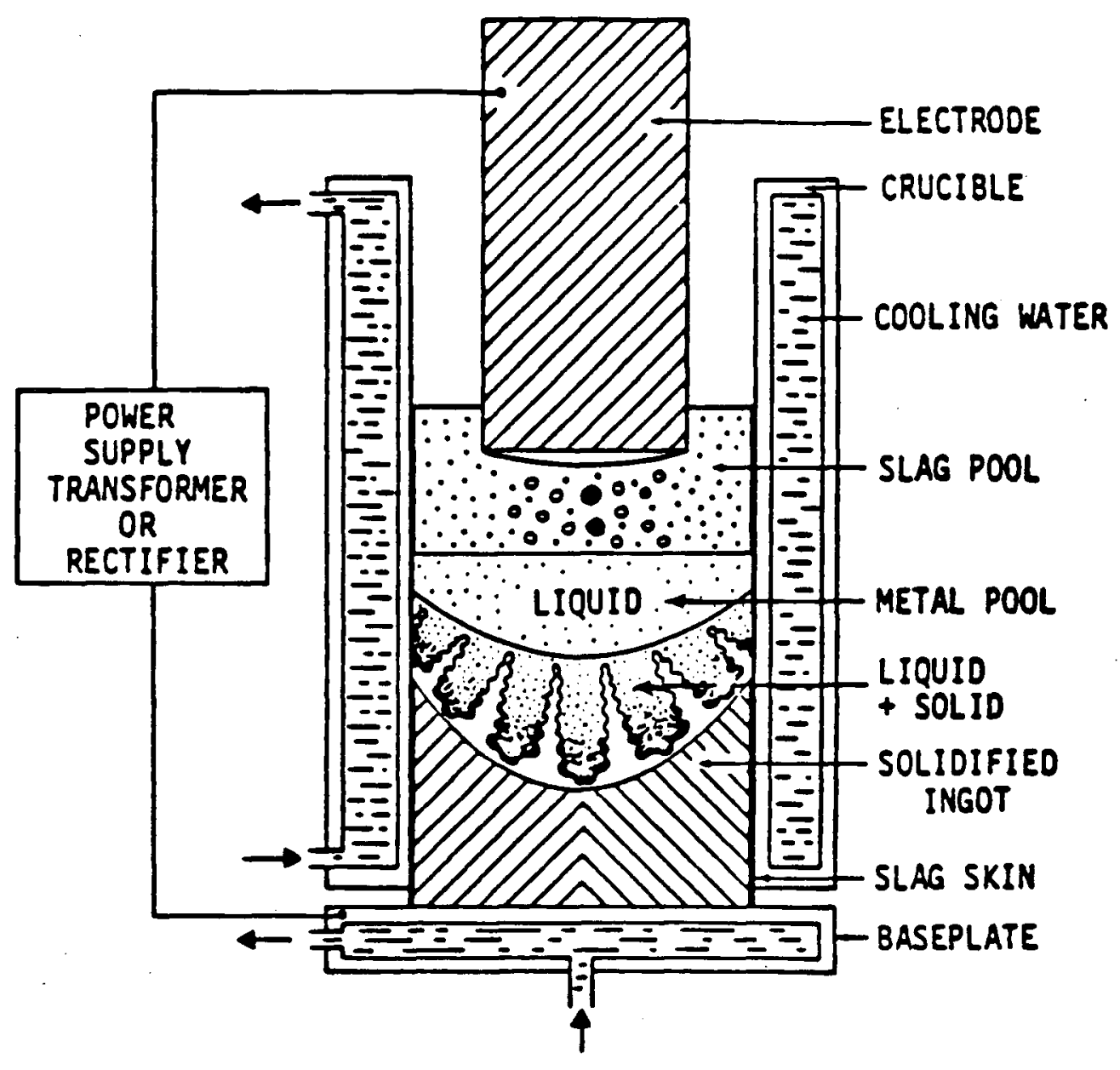

Figure 1.1: Schematic illustration of an ESR furnace. 


\section{Magnetic fields in Metals Processing}

$\underline{\text { Efrect }}$

THERMAL _— Induction melting

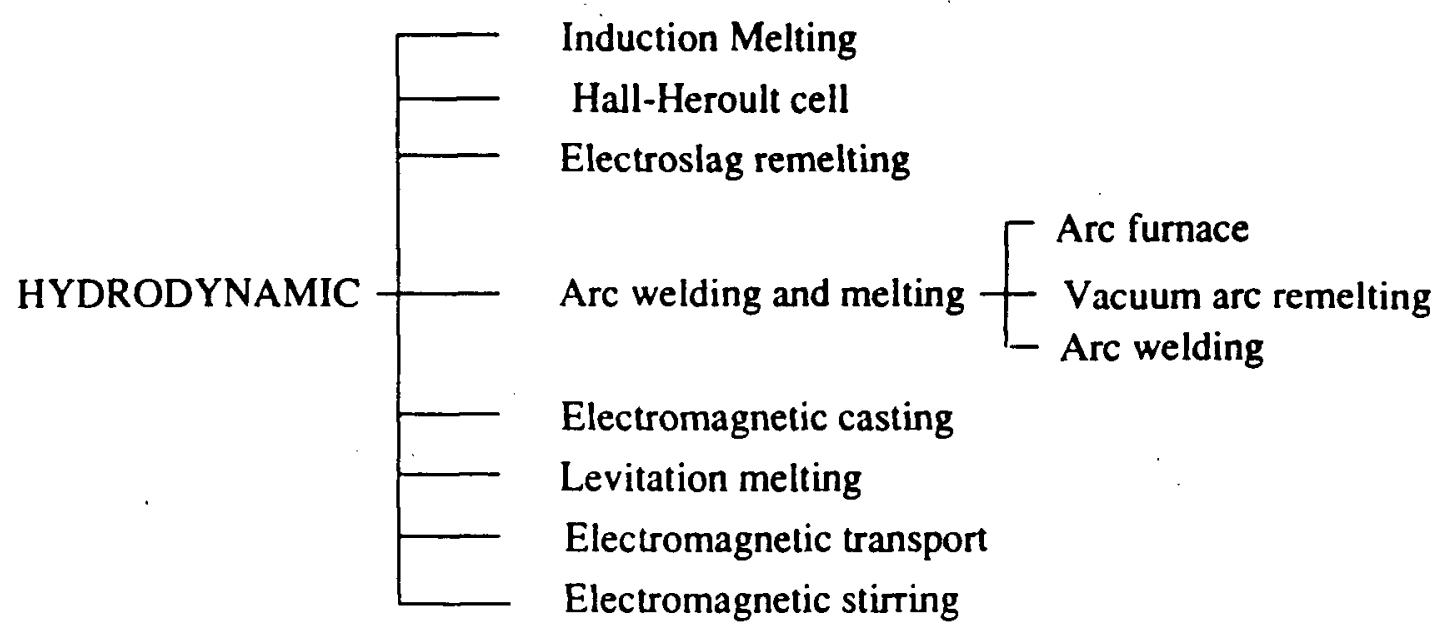

Figure 1.2: Metallurgical applications of EMS. 


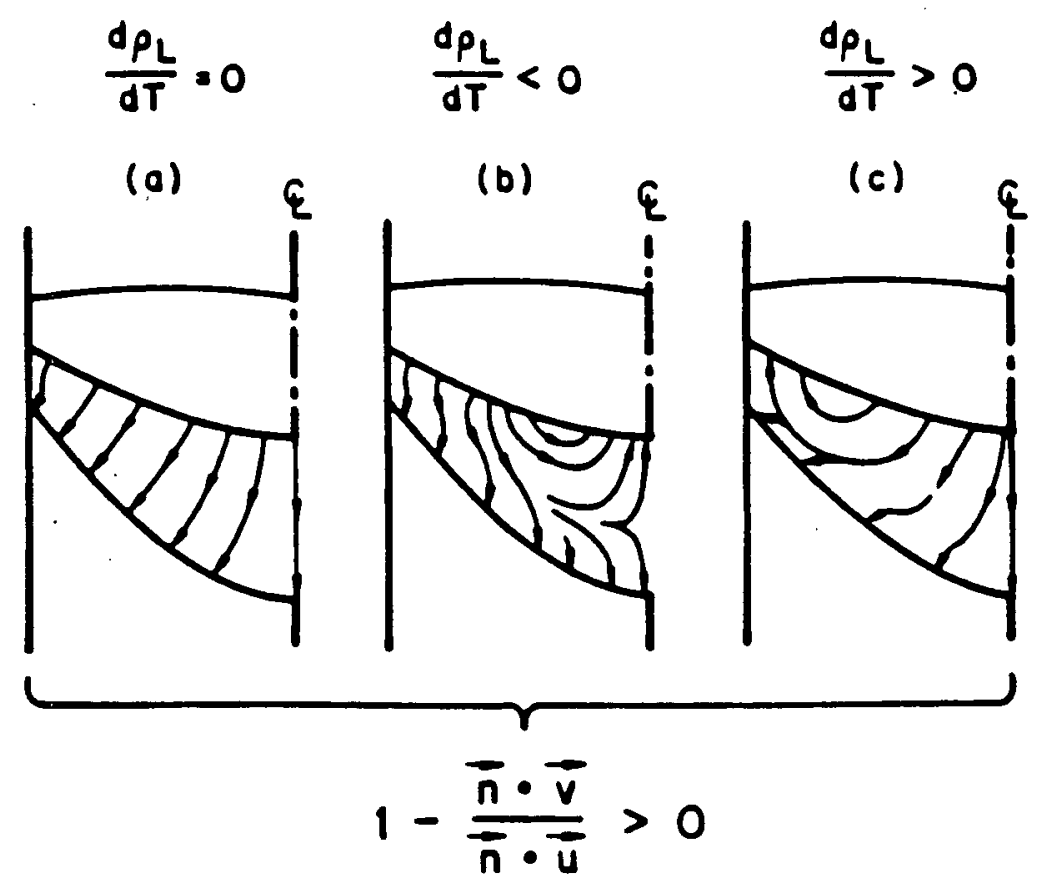

Figure 1.3: Schematic illustration of possible interdendritic fluid flow in axisymmetric ingots. (a) Flow resulting in negative segregation at ingot centerline, (b) flow resulting in positive segregation, (c) flow resulting in enhanced negative segregation [104]. 


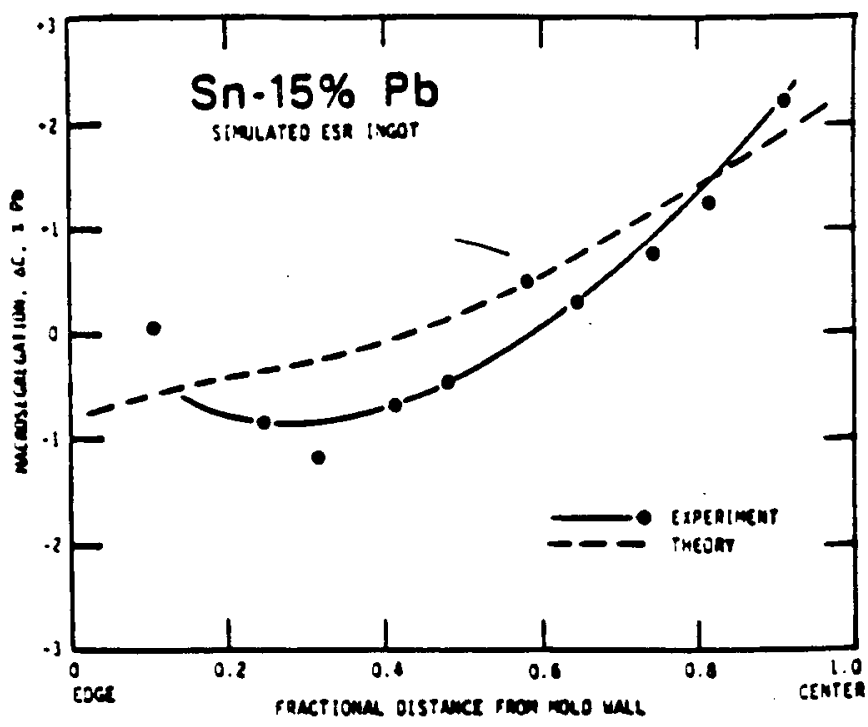

(a)

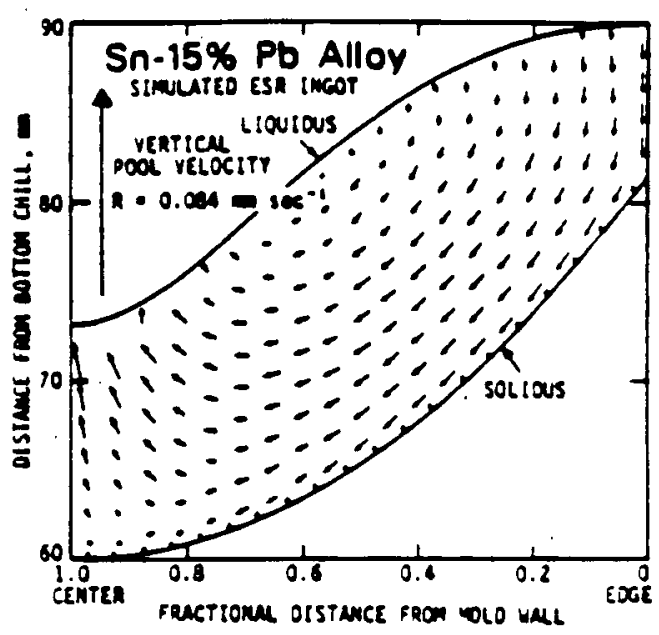

(b)

Figure 1.4: (a) Comparison of experimental and theoretical segregation profiles in a $\mathrm{Sn}-15 \% \mathrm{~Pb}$ ingot produced in a simulated ESR system, (b) calculated interdendritic flow velocities [100]. 


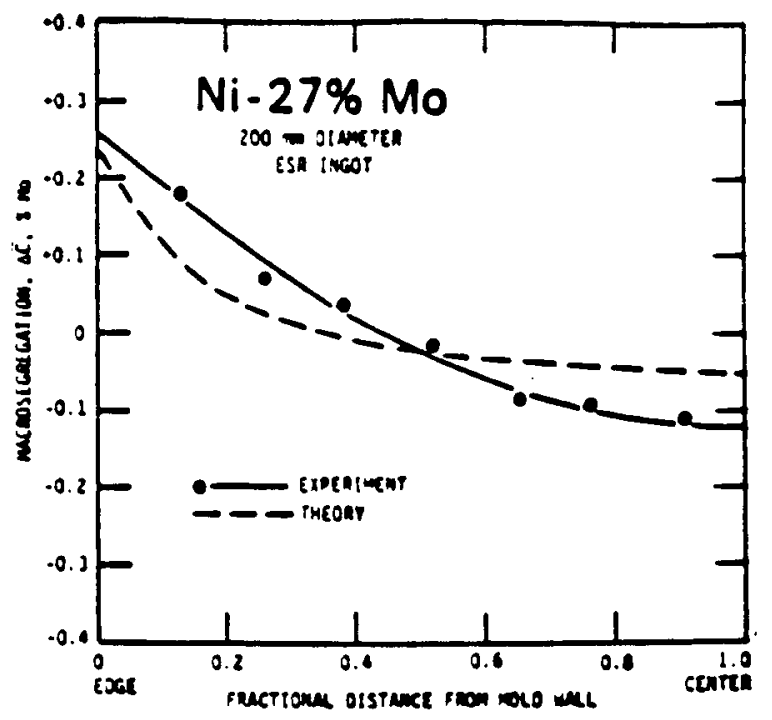

(a)

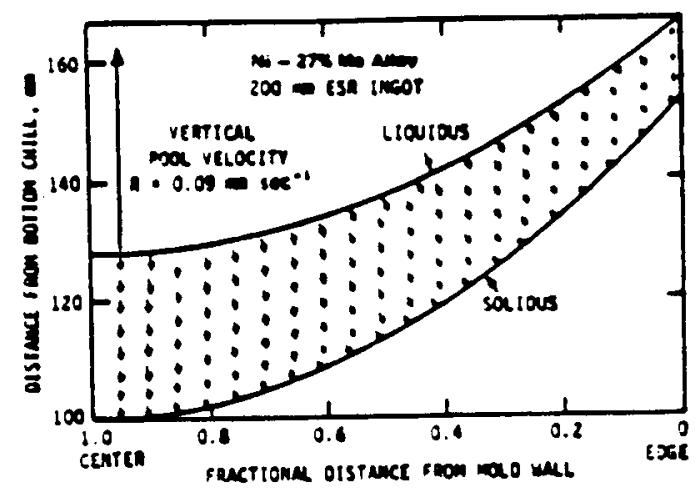

(b)

Figure 1.5: (a) Comparison of experimental and theoretical segregation profiles in a $\mathrm{Ni}-27 \% \mathrm{Mo}, 200 \mathrm{~mm}$ dia., ESR ingot, (b) calculated interdendritic flow velocities [100]. 


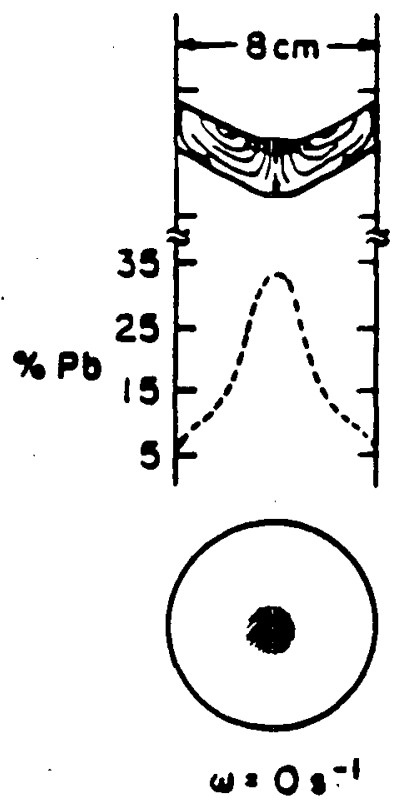

(a)
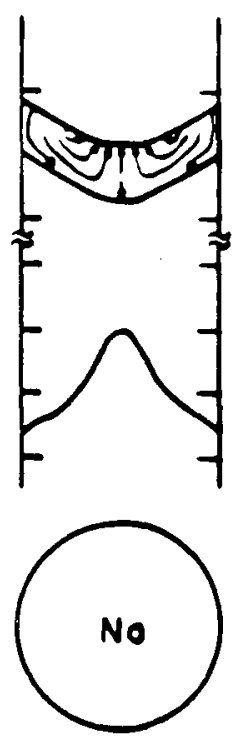

$\omega=8.7 \mathrm{~s}^{-1}$

(b)
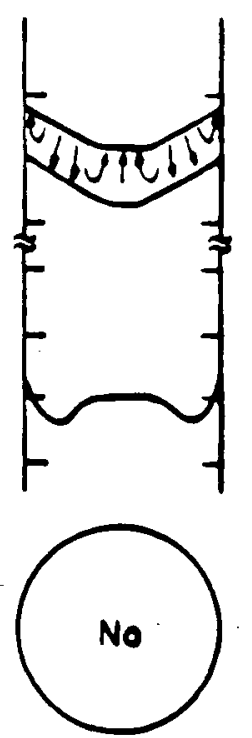

$\omega=13 s^{-1}$

(c)
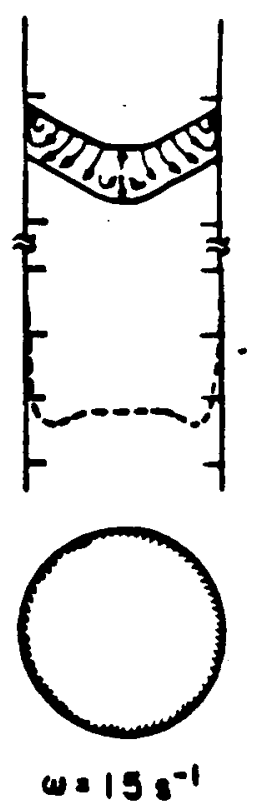

(d)

Figure 1.6: Effect of rotation of mould on flow of interdendritic liquid and macrosegregation in $\mathrm{Sn}-12 \% \mathrm{~Pb}$ ingots. Freckles are expected along the center in (a) and at the surface in (d) [102]. 


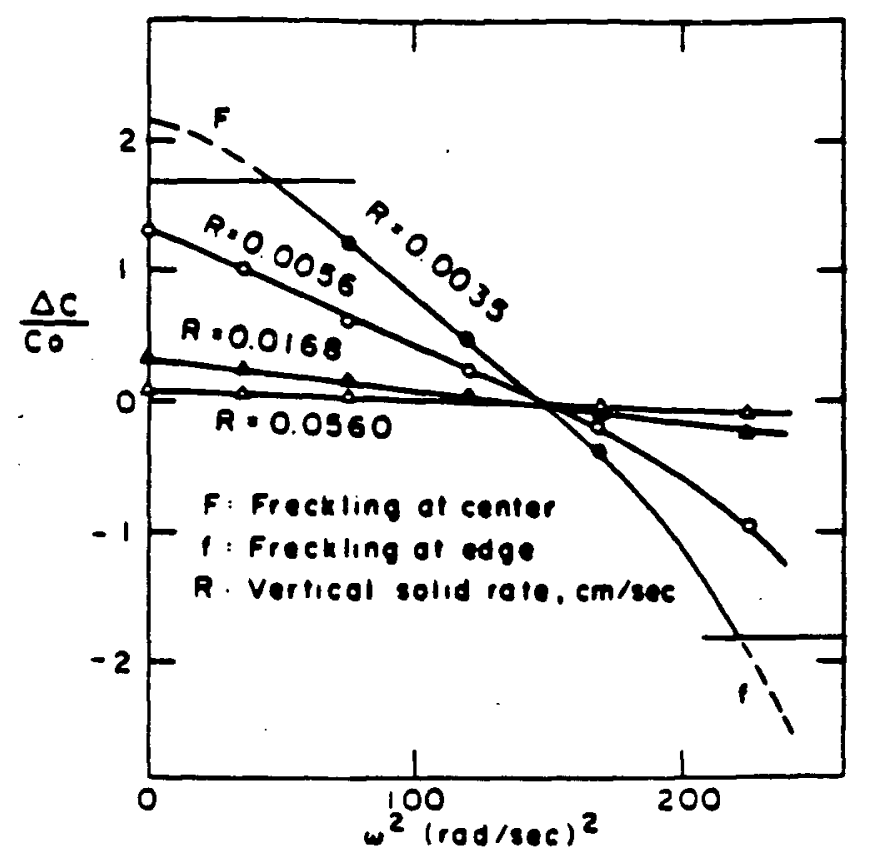

Figure 1.7: Difference in composition between center and surface as a function of rotational speed and solidification rate for $\mathrm{Sn}-12 \% \mathrm{~Pb}$ ingots [102]. 

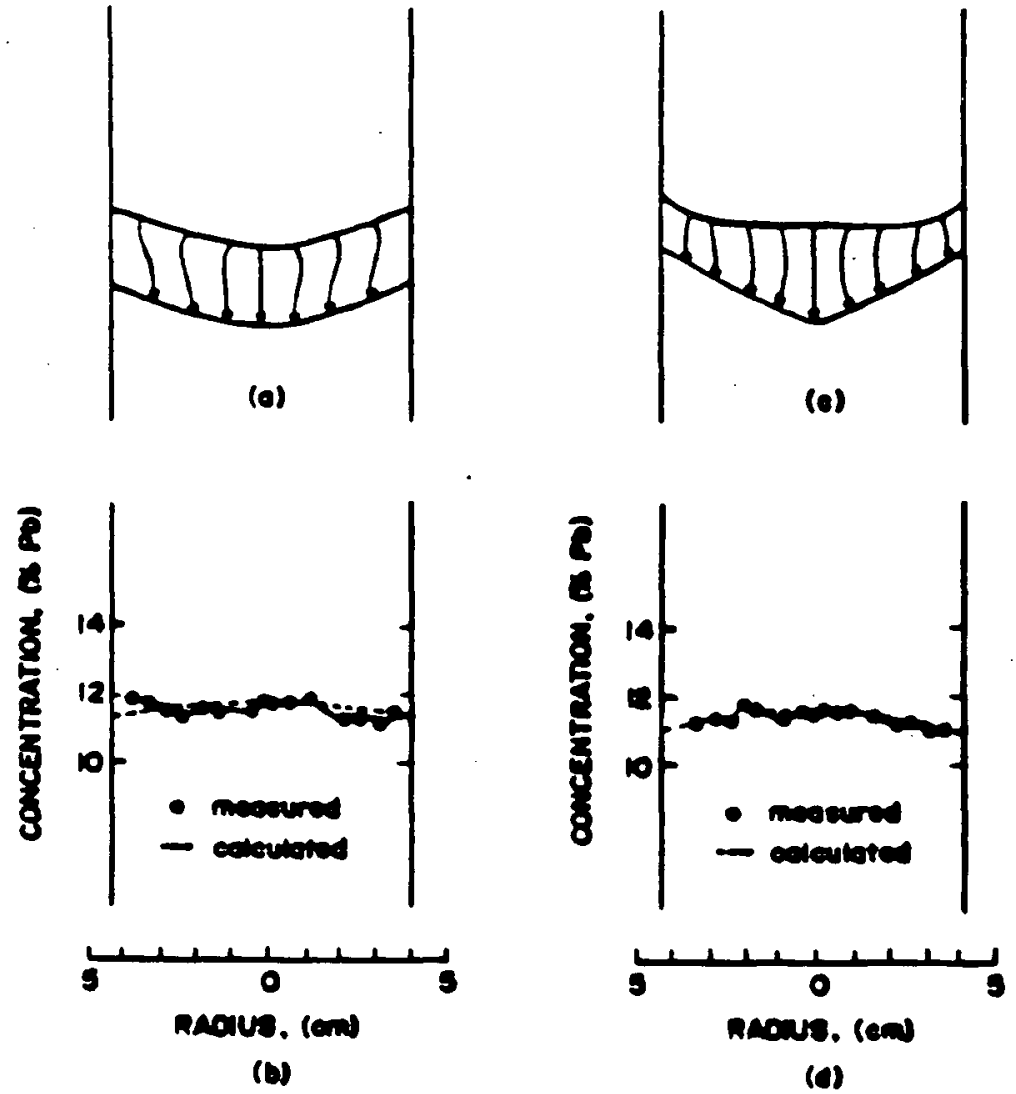

Figure 1.8: Flow patterns and macrosegregation: (a) calculated flow pattern in lower half (0 rpm), (b) measured and calculated macrosegreagtion of the lower half, (c) calculated flow pattern in the upper half $(23 \mathrm{rpm}),(\mathrm{d})$ measured and calculated macrosegregation of the upper half [102]. 


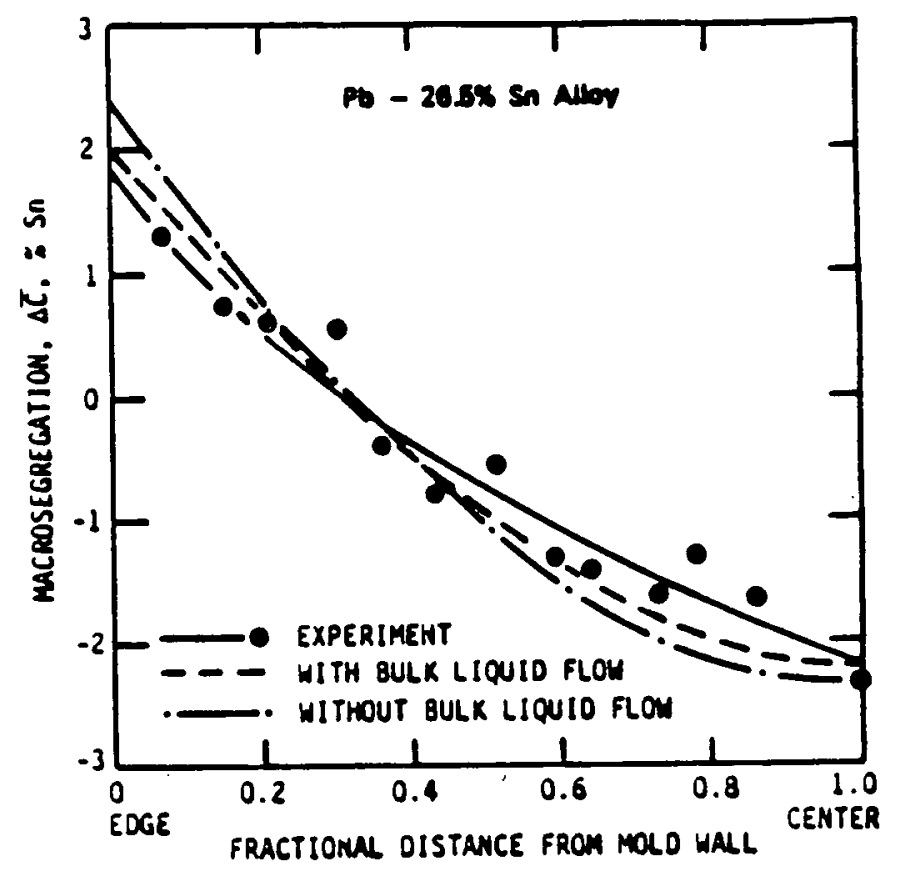

Figure 1.9: Comparison between experimental and theoretical segregation profiles in a $\mathrm{Pb}-26.5 \% \mathrm{Sn}$ ingot [104]. 


\section{Chapter 2}

\section{Mathematical Simulation}

A theoretical analysis of the electromagnetic stirring of ESR ingots was conducted in order to establish a reference frame for the experimental programme as well as to help in the analysis of the results. Two exercises were carried out : 1) The simulation of the thermal field during the remelting, by modifying an existing computer code, and 2) the calculation of the electromagnetic body force distribution generated by the externally applied field.

\subsection{Thermal Regime}

Numerous models of the thermal history for both, slag and metal pool, have been reported [120 - 130]. In general, one can classify them in two groups : 1) those in which heat transfer and fluid flow phenomena are coupled through an effective thermal conductivity in the liquid metal $[120-126]$ and 2) those which involve the simultaneous solution of the equations of motion and energy [127 - 130]. In the latter case a model for describing the turbulence characteristics of the fluid, usually the $k-W$ or $k-\epsilon$ models, has to be incorporated.

It should be pointed out that although the second approach is more rigorous (resulting in a detailed description of the fluid flow) a careful characterization of the boundary conditions as well as of the removal of latent heat is of upmost importance.

A previously developed code [131] was used to simulate the thermal regime when EMS is applied during ESR. This particular model considers steady-state conditions and 
links the heat transfer phenomena in the slag and the metal pool through a predefined temperature distribution at the top of the ingot. The convective flow in the pool is taken into account by increasing the value of the thermal conductivity in that zone while the latent heat evolution is incorporated by using a modified heat capacity. The numerical technique used is finite differences and a typical mesh will include 11 nodes in the radial direction and 101 nodes in the axial direction. The nodes are regularly spaced in the whole domain of the computation.

The original code was developed based on a heat balance over each volume element as shown in fig. 2.10. Since the convective and conductive flows are in parallel, they were added in a combined transport mechanism characterized by an effective thermal conductivity. The modifications implemented are as follow : 1) in order to simulate the flow of heat in preferential directions the thermal conductivity was treated as a vectorial quantity by introducing a multiplying factor and 2) this anisotropy of the thermal conductivity was restricted to nodes close to the center of the ingot only.

\subsection{Electromagnetic Body Force}

The distribution of electromagnetic body forces plays an important role in determining the fluid flow patterns and, therefore, the thermal history, mass transfer and mechanical interactions taking place during processes involving magnetic fields. Because of the multiple choices of electrical configurations capable of producing magnetic fields of different intensities and distributions, a predictive tool that can be used for designing purposes is often desirable.

Since the magnetic Reynolds number is small for most of the metallurgical applications the Navier-Stokes equations can be decoupled from the magnetic transport equation. Then, the first step in order to calculate the magnetic force contribution to the fluid 
motion is to evaluate the induced magnetic field intensity distribution. In the ESR process there is a self-induced magnetic field generated by the passage of the melting current through the electrode. Therefore, the total electromagnetic body force would be composed by the interaction of both the self-induced and the externally applied magnetic fields with the melting current. Assuming that the magnitude of the latter is greater than the one of the former, the instantaneous electromagnetic body force is calculated as

$$
\vec{F}_{m}=\vec{J} \times \vec{B}_{\mathrm{ext}}
$$

The current density distribution in both the slag and the metal pool is still a matter of discussion. The reason for this is that an unknown part of the current is carried by the mould which modifies the boundary condition at the mould wall required for solving the Maxwell's equations. In this work, the steady-state current distribution computed by Jardy et al. [132] is used as an input.

The electromagnetic field distribution created by the stirring device is calculated following work by Gagnoud et al. [133] for levitation melting using cold crucibles. The setup is analogous to a transformer : the induction coil is the equivalent of the primary winding and the mould-charge system (where charge is both the metal pool and the slag) constitutes the secondary.

During AC operations current varies periodically with time and so does the induced magnetic field. Therefore, the unsteady-state term in eq. 1.7 cannot be neglected. A mathematical technique commonly used to handle this situation is the introduction of 'phasors'. A phasor is essentially a vector that rotates in the real-imaginary plane at a frequency of $\omega$ radians. The usefulness of this approacli consists in that the real part of a phasor, in the mathematical sense, corresponds to the real component in the physical sense. The introduction of phasors obviates the unsteady character of the problem at 
the cost of introducing complex quantities. Another mathematical entity, the vector potential (see p. 6) is also used in the solution of eq. 1.7.

The presence of the conducting crucible and charge as well as the fluctuating nature of the induced field makes this a very difficult problem if an analytical solution is sought. Consequently, a numerical solution was implemented.

The first step in building the numerical algorithm is the computation of the vector potential induced by a current flowing in a circular path. For this geometry the vector potential, $\vec{A}$, induced by a current flowing through a wire of radius $\rho$ and positioned at $z=\xi$ (see fig. 2.11) has the components

$$
\vec{A}=\left(0, A_{\theta}, 0\right)
$$

The azimuthal component induced by a single turn coil of infinitesimal area is defined by

$$
A_{\theta}(r, z)=\frac{\mu_{o}}{\pi} I f(\rho, \xi, r, z)
$$

where

$$
f(\rho, \xi, r, z)=\sqrt{\frac{\rho}{m r}}[(1-m / 2) K(m)-E(m)]
$$

and

$$
m=\frac{4 \rho r}{(r+\rho)^{2}+(z-\xi)^{2}}
$$

In eq. 2.32 the quantities $K(m)$ and $E(m)$ are elliptic integrals of the first and second kind.

This expression can be extended to a wire of finite dimensions positioned at any angle with respect to the $\mathrm{r}-\theta$ plane :

$$
A_{\theta}=\frac{\mu_{o}}{\pi} \int_{n} \int_{t} J(t) \exp \left(-\frac{(1+\jmath) n}{\delta}\right) g(n, t) \mathrm{d} t \mathrm{~d} n
$$


where

$$
\begin{array}{r}
g(n, t)=f(\rho, \xi, r, z) \\
\jmath=\sqrt{-1}
\end{array}
$$

and $\delta$ is the magnetic skin depth.

When $h$ is small enough to assure that the current density is uniform in each element it is possible to write

$$
J(t)=\text { const }=J_{i}
$$

and

$$
I_{i}=J_{i} \delta h
$$

Finally, since the skin depth is small compared with the radius of the mould,

$$
A_{\theta}(r, z)=\frac{\mu_{o} \delta h}{2 \pi(1+\jmath)} J_{i}\left[f\left(\rho, \xi+\frac{h}{2}, r, z\right)+f\left(\rho, \xi-\frac{h}{2}, r, z\right)\right]
$$

The model used to simulate the stirring assembly is shown in fig. 2.12. The exciting current induces two mutually dependent current sheets at the internal and external faces of the mould as well as a current sheet at the outer surface of the charge. Each one of these current sheets has a width of the order of the corresponding magnetic skin depth. For the numerical simulation all of these sheets are divided into a number of elementary wires of height $h$ and a uniform current distribution across each element is assumed. For such a model the induced vector potential at a particular position will be given by the contributions of the exciting coil, the composite mould and the charge. 
Then, the vector potential induced by the system coil + mould + charge along the wire $k$ of the external current sheet of the crucible is

$$
\begin{array}{r}
A_{\theta}\left(R_{e}, Z_{e}(k)\right)=\frac{\mu_{o} \delta h}{2 \pi(1+\jmath)}\left\{\sum _ { i = 1 } ^ { N _ { i } } J ( R _ { e } , Z _ { e } ( i ) ) \left[f\left(R_{e}, Z_{e}(i)+\frac{h}{2}, R_{e}, Z_{e}(k)\right)\right.\right. \\
\left.\left.+f\left(R_{e}, Z_{e}(i)-\frac{h}{2}, R_{e}, Z_{e}(k)\right)\right]\right\} \\
+\frac{\mu_{o} \delta h}{2 \pi(1+\jmath)}\left\{\sum _ { i = 1 } ^ { N _ { i } } J ( R _ { i } , Z _ { i } ( i ) ) \left[f\left(R_{i}, Z_{i}(i)-\frac{h}{2}, R_{e}, Z_{e}(k)\right)\right.\right. \\
\left.\left.+f\left(R_{i}, Z_{i}(i)-\frac{h}{2}, R_{e}, Z_{e}(k)\right)\right]\right\} \\
+\frac{\mu_{o}}{\pi} I_{\operatorname{coil}}\left\{\sum_{m=1}^{N} f\left(R, Z(m), R_{e}, Z_{e}(k)\right)\right\} \\
+\frac{\mu_{o} \delta_{c} h}{2 \pi(1+\jmath)}\left\{\sum _ { i = 1 } ^ { N _ { i } } J ( R _ { c } , Z _ { c } ( i ) ) \left[f\left(R_{c}, Z_{c}(i)+\frac{h}{2}, R_{e}, Z_{e}(k)\right)\right.\right. \\
\left.\left.+f\left(R_{c}, Z_{c}(i)-\frac{h}{2}, R_{e}, Z_{e}(k)\right)\right]\right\}
\end{array}
$$

In order to solve eq. 2.40 a relationship between sheet pairs has to be established. This can be done by defining the current density vector in terms of the vector potential and the electrical potential :

$$
\vec{J}=-\sigma \operatorname{grad} \vec{V}-\jmath \omega \sigma \vec{A}
$$

As a first approximation, the induced currents are assumed to be flowing along purely horizontal loops. Mathematically, this closure of the current is expressed as

$$
J\left(R_{e}, Z_{e}(i)\right)=-J\left(R_{i}, Z_{i}(i)\right)
$$

This results in a constant electrical potential around the system, or

$$
\operatorname{grad} \vec{V}=0
$$


Therefore, the vector potential and current density at a given element in the external sheet are related through

$$
J\left(R_{e}, Z_{e}(i)\right)=-\jmath \omega \sigma A\left(R_{e}, Z_{e}(i)\right)
$$

Similarly,

$$
J\left(R_{i}, Z_{i}(i)\right)=-\jmath \omega \sigma A\left(R_{i}, Z_{i}(i)\right)
$$

and

$$
J\left(R_{c}, Z_{c}(i)\right)=-\jmath \omega \sigma A\left(R_{c}, Z_{c}(i)\right)
$$

for the internal and charge current sheets, respectively.

Finally, substituting eqns. 2.44 to 2.46 in eq. 2.40 results in :

$$
\begin{array}{r}
A_{\theta}\left(R_{e}, Z_{e}(k)\right)=\frac{\mu_{o} \delta h}{2 \pi(1+\jmath)}\left\{\sum_{i=1}^{N_{i}}-\jmath \omega \sigma_{m} A_{\theta}\left(R_{e}, Z_{e}(i)\right)\left[f\left(R_{e}, Z_{e}(i)+\frac{h}{2}, R_{e}, Z_{e}(k)\right)\right.\right. \\
\left.\left.+f\left(R_{e}, Z_{e}(i)-\frac{h}{2}, R_{e}, Z_{e}(k)\right)\right]\right\} \\
+\frac{\mu_{o} \delta h}{2 \pi(1+\jmath)}\left\{\sum _ { i = 1 } ^ { N _ { i } } \jmath \omega \sigma _ { m } A _ { \theta } ( R _ { e } , Z _ { e } ( i ) ) \left[f\left(R_{i}, Z_{i}(i)-\frac{h}{2}, R_{e}, Z_{e}(k)\right)\right.\right. \\
\left.\left.+f\left(R_{i}, Z_{i}(i)-\frac{h}{2}, R_{e}, Z_{e}(k)\right)\right]\right\} \\
+\frac{\mu_{o}}{\pi} I_{\operatorname{coil}}\left\{\sum_{m=1}^{N} f\left(R, Z(m), R_{e}, Z_{e}(k)\right)\right\} \\
+\frac{\mu_{o} \delta_{c} h}{2 \pi(1+\jmath)}\left\{\sum_{i=1}^{N_{i}}-\jmath \omega \sigma_{c} A_{\theta}\left(R_{c}, Z_{c}(i)\right)\left[f\left(R_{c}, Z_{c}(i)+\frac{h}{2}, R_{e}, Z_{e}(k)\right)\right.\right. \\
\left.+f\left(R_{c}, Z_{c}(i)-\frac{h}{2}, R_{e}, Z_{e}(k)\right)\right\}
\end{array}
$$


Similarly, the vector potential induced in the charge is given by

$$
\begin{array}{r}
A_{\theta}\left(R_{c}, Z_{c}(k)\right)=\frac{\mu_{o} \delta h}{2 \pi(1+\jmath)}\left\{\sum_{i=1}^{N_{i}}-\jmath \omega \sigma_{m} A_{\theta}\left(R_{e}, Z_{e}(i)\right)\left[f\left(R_{e}, Z_{e}(i)+\frac{h}{2}, R_{c}, Z_{c}(k)\right)\right.\right. \\
\left.\left.+f\left(R_{e}, Z_{e}(i)-\frac{h}{2}, R_{c}, Z_{c}(k)\right)\right]\right\} \\
+\frac{\mu_{o} \delta h}{2 \pi(1+\jmath)}\left\{\sum _ { i = 1 } ^ { N _ { i } } \jmath \omega \sigma _ { m } A _ { \theta } ( R _ { e } , Z _ { e } ( i ) ) \left[f\left(R_{i}, Z_{i}(i)-\frac{h}{2}, R_{c}, Z_{c}(k)\right)\right.\right. \\
\left.\left.+f\left(R_{i}, Z_{i}(i)-\frac{h}{2}, R_{c}, Z_{c}(k)\right)\right]\right\} \\
+\frac{\mu_{o}}{\pi} I_{\operatorname{coil}}\left\{\sum_{m=1}^{N} f\left(R, Z(m), R_{c}, Z_{c}(k)\right)\right\} \\
+\frac{\mu_{o} \delta_{c} h}{2 \pi(1+\jmath)}\left\{\sum_{i=1}^{N_{i}}-\jmath \omega \sigma_{c} A_{\theta}\left(R_{c}, Z_{c}(i)\right)\left[f\left(R_{c}, Z_{c}(i)+\frac{h}{2}, R_{c}, Z_{c}(k)\right)\right.\right. \\
\left.\left.+f\left(R_{c}, Z_{c}(i)-\frac{h}{2}, R_{c}, Z_{c}(k)\right)\right]\right\}
\end{array}
$$

Eqns. 2.47 and 2.48 constitute a system of $2 N_{i} \times 2 N_{i}$ linear simultaneous equations of complex coefficients. In order to avoid dealing with complex numbers these equations were splitt into their real and imaginary parts which results in two systems of $2 N_{i} \times 2 N_{i}$ simultaneous equations of real coefficients. These systems can be readily solved using any of the available matrix solver packages. Particularly, the SLIMP package available in the AHMDAL library at UBC was utilized.

In transforming the coefficients of eqns. 2.47 and 2.48 from complex to real, the following definitions were used :

$$
A_{\text {real }}=\operatorname{Re}\left\{\left(A_{r}+\jmath A_{j}\right) e^{i w t}\right\}
$$

and

$$
I_{\text {real }}=\operatorname{Re}\left\{I_{o} e^{i w t}\right\}
$$

Once eqns. 2.47 and 2.48 are solved the vector potential distribution at every point of 
the system can be computed. Then, the induced electromagnetic field is readily calculate using eq. 1.13.

Finally, the interaction of the induced magnetic field with the current passing through the system gives the instantaneous electromagnetic body force which has only one component :

$$
F_{\theta}(r, z)=-\left(B_{z}(r, z) J_{r}(r, z)-B_{r}(r, z) J_{z}(r, z)\right)
$$




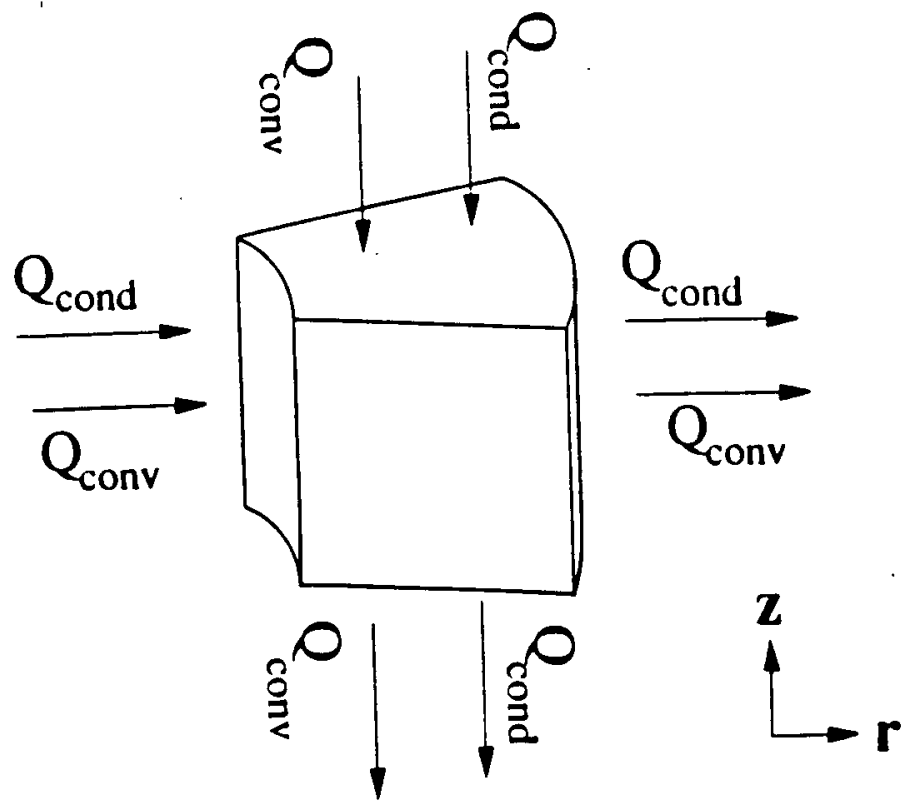

(a)

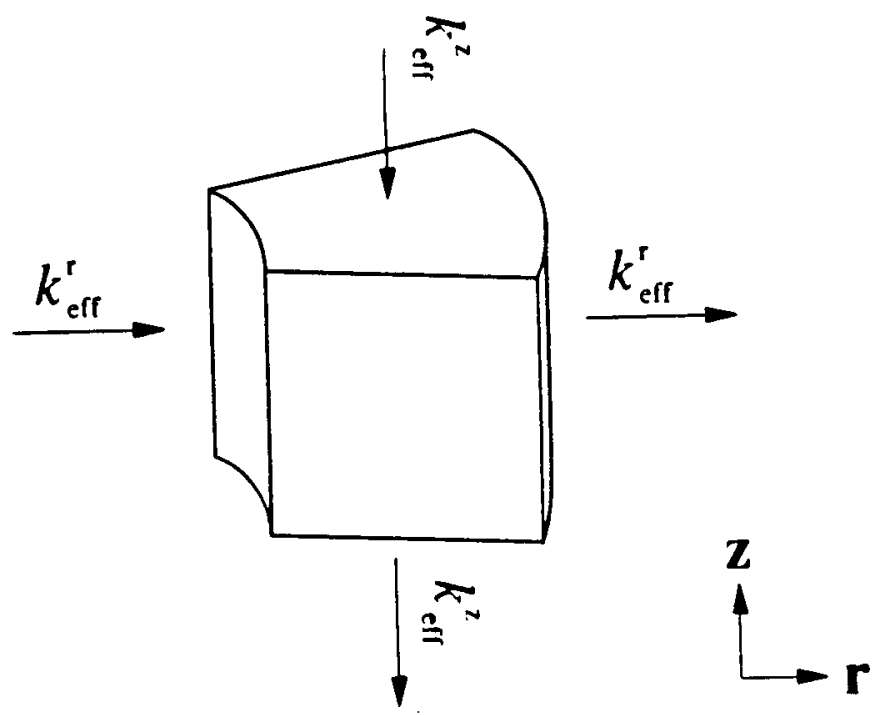

(b)

Figure 2.10: Heat balance over a volume element. (a) Isotropic $k_{\text {eff }}$, (b) anisotropic $k_{\text {eff }}$. 


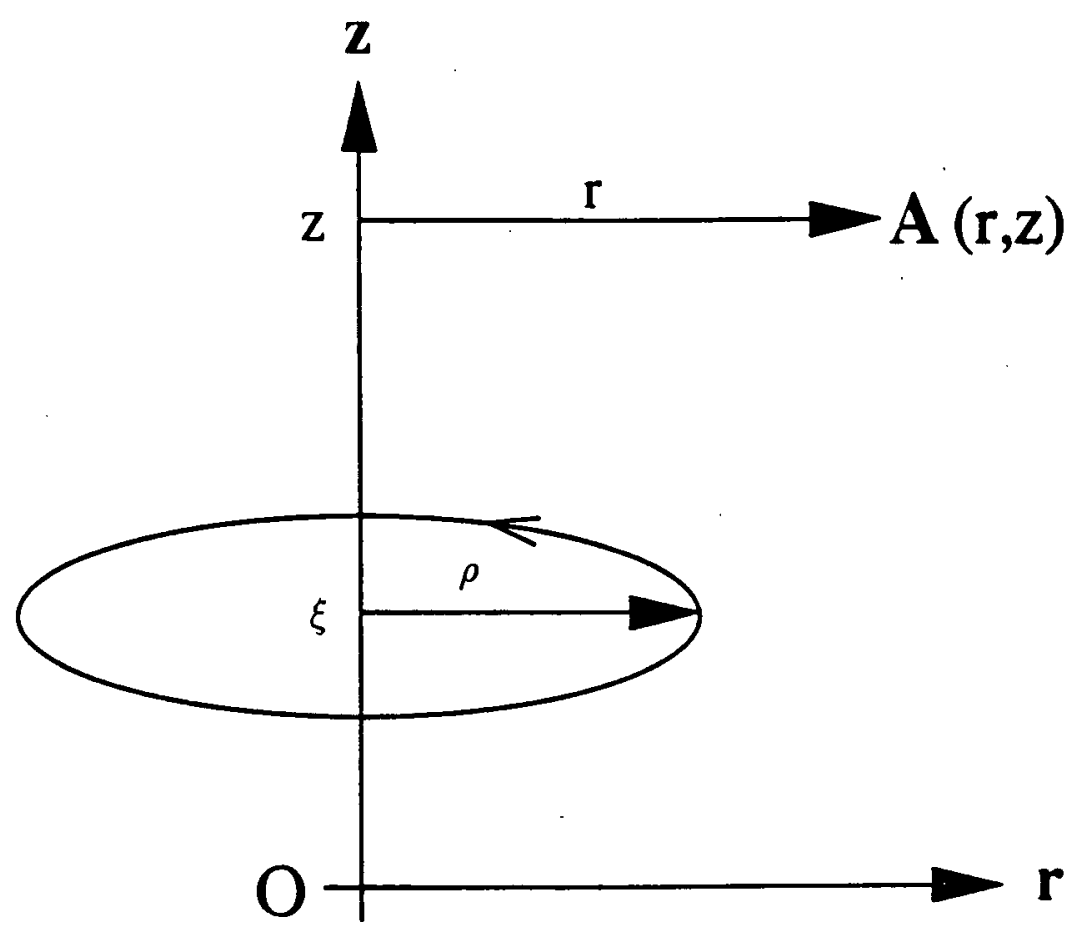

Figure 2.11: Vector potential induced by current flowing through a circular path. 


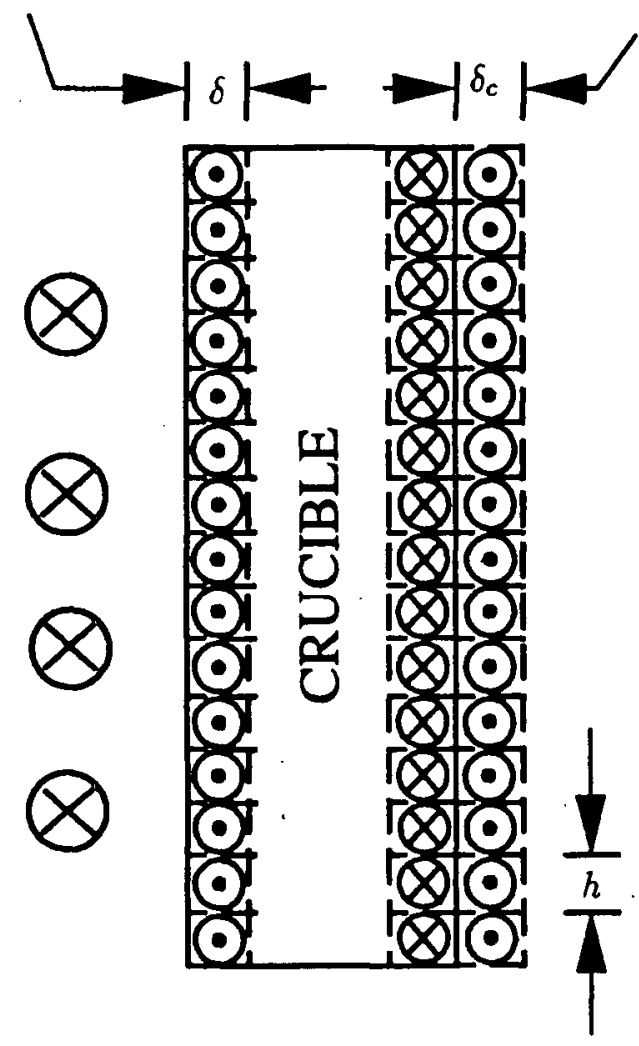

Figure 2.12: Model used to calculate magnetohydrodynamic quantities. 


\section{Chapter 3}

\section{Experimental Procedures}

\subsection{Experimental Setup}

The laboratory-scale ESR unit at UBC was used to conduct the entire experimental programme. A detailed description of this particular unit can be found elsewhere [134].

Stirring of the system slag-liquid pool was accomplished through the interaction of an external electromagnetic field and the melting current passing through the ESR furnace. Since the main unit operates with AC (at a frequency of $60 \mathrm{~Hz}$ ), the current used to feed the external coil had to be AC too (see table 1.1). In order to have as much flexibility as possible an independent source was used to provide the exciting current : for low currents a Hobart welder model T-500 was employed while an AC power supply was used when high currents (over $300 \mathrm{~A}$ ) were needed.

In order to design the most effective mould one has to consider the magnetic skin effect (see p. 7). The magnetic field damping caused by shielding of the field lines is illustrated in fig. 3.13. In this figure the ratio of the magnetic field intensity at a given distance from the source to its original value is plotted against the distance from the surface of a block, for materials with low and high electrical resistivity. A typical ESR mould used at UBC is made of aluminium and has a thickness of $11 / 2$ in. Refering to fig. 3.13 the magnitude of an externally applied AC magnetic field at the inner surface of the mould would have decayed to $\sim 3 \%$ of its original value. In order to overcome this loss of magnetic field intensity a mould made of a material of very high electrical resistivity 
would be desirable. Unfortunately, the heat extraction requirements of the process [135] ask for a mould capable of extracting a considerable amount of heat, which requires a good thermal conductor. In consequence a new mould design had to be considered.

In first instance an attempt was made to use a copper bar wound in a cylindrical shape with a double purpose : 1) to act as a magnetic field source and,2) to serve as the mould itself. Cooling was accomplished by running water between the outer surface of the 'mould' and a rubber hose. After several trials it was not possible to prevent water leaking and therefore it was decided to take a different approach. In the inductoslag process [136] an split cold crucible is used to contain the melt, the idea behind the split crucible being the possibility of getting magnetic field lines to cross the melt by leaking through the splits. It was thought that a similar design could be applied to an ESR mould.

An schematic view of the stirring mould is shown in fig. 3.14. Each half of a 4 in dia., 6 in. high, standard aluminium mould was split into three sections, each one containing a water inlet and outlet, and solid graphite pieces (fig. 3.15) were used to replace the original material. Once the aluminium and graphite blocks were clamped together a 4 turn coil made from $1 / 2$ in. O.D. stainless steel tube was placed around the composite mould. In order to prevent electric arcs the coil was coated with epoxy paint and rubber pieces where placed between the coil and the mould. A detail of the final arrangment is shown in fig. 3.16. Before an experiment was conducted the inside surface of the mould was painted with boron nitride paint (type $S$ ).

The mould configuration consisted of two standard aluminium moulds placed at the bottom and top of the stirring mould. The bottom mould was 3 in. in dia., 4 in. high and was used as an starter while a 5 in. dia., 16 in. high mould was used to finish the experiment. Copper leads were connected from the main power source to both bottom and top moulds which can, therefore, be considered as live moulds. 
A photograph of the complete setup is shown in fig. 3.17.

\subsection{Experimental Programme}

The remelting experiments are summarized in table 3.2 , complemented by table 3.3 which includes the chemical composition of the several slags used in the experiments. For all the runs, the electromagnetic stirring was started once the liquid metal had reached the stirring mould, as determined through monitoring the outlet mould water temperatures. The stirrer was turned on for times ranging from 5 to 150 seconds in the unsteady-state trials, although in most of the cases the time for stirring was in the 40 to 60 second range (a typical ESR run takes about $25 \mathrm{~min}$ ).

Experiment I was conducted in order to visually inspect the slag top surface with and without stirring. For this purpose the layer of fiberfrax normally placed on top of the upper mould was removed. Experiments II to VII were used to establish the effect of different exciting currents levels on the resulting structure as well as on the thermal regime (through the isotherms). Both steady and unsteady cases were studied. For run VII the stirrer was first turned on for $60 \mathrm{sec}$, stopped for $50 \mathrm{sec}$ and then turned on for the rest of the experiment. The thermal profile resulting during the steady-state stirring was traced using tungsten powder $(-100+170$ mesh). The effect of a different electrode material was investigated via experiments VIII $(\mathrm{Cu}-50 \% \mathrm{Fe}$ ), and IX and X (Inconel 718). Experiments IX and $X$ also provided a reference to compare the remelting of the same electrode under two slags of different chemical composition.

In order to study the mixing conditions prevailing in the pool for both, stirred and unstirred ingots, two experiments using a chemical tracer were conducted (runs XI and XII). The electrode was a $11 / 2$ " dia. round bar of standard 4340 alloy steel (see table 3.4 for composition). Since any joint would perturb the fluid flow in the pool the electrodes 
used for these experiments were made with one single piece. In both cases $10 \mathrm{~g}$ of tungsten powder $(-100+170$ mesh) were throughly mixed with $9 \mathrm{~g}$ of nickel powder $(+150$ mesh $)$ and added $870 \mathrm{~s}$ (6100 revs) after an stable condition had been reached.

In all the cases the melting current, inlet and outlet temperatures as well as electrode travel and electrical parameters were monitored and recorded once the melt had reached an stable condition.

\subsection{Analysis}

For all the experiments a longitudinal section of the remelted ingots was obtained and prepared for macrography. For the 4340 alloy steel ingots standard hot acid etch $\left(50 \% \mathrm{HCl}\right.$ in water, at $\left.70^{\circ} \mathrm{C}\right)$ was used; in the case of Inconel 718 and $\mathrm{Cu}-50 \% \mathrm{Fe}$, Marble's reagent $\left(4 \mathrm{~g} \mathrm{CuSO}_{4}, 50 \mathrm{ml} \mathrm{HCl}, 50 \mathrm{ml} \mathrm{H}_{2} \mathrm{O}\right.$ ) was employed.

A sample comprising the beginning and end of the stirring zone in experiment II was prepared for microscopic examination. The sample was obtained at the centreline of a longitudinal section of the ingot and etched with Oberhoffer's reagent ( $30 \mathrm{~g} \mathrm{FeCl}_{3}$, $1 \mathrm{~g} \mathrm{CuCl}_{2}, 0.5 \mathrm{~g} \mathrm{SnCl}_{2}, 50 \mathrm{ml} \mathrm{HCl}, 500 \mathrm{ml}$ ethyl alcohol, $500 \mathrm{ml}$ distilled water ). A wave dispersive $\mathrm{x}$-ray (WDX) line scan of a segregated region was performed using the microspec WDX-2A system equipped with WDX-3PC software. The analysis was done at a magnification of $110 \mathrm{X}$ using an accelerating voltage of $20 \mathrm{kV}$ and a $15 \% \mathrm{P}, 85 \%$ $\mathrm{Cu}$ standard.

Four samples (see fig. 3.18) were obtained from ingot VII at a position where steady electromagnetic stirring was started (as revealed by the macroetch). The samples represented the three orthogonal directions plus a plane perpendicular to the tungsten-marked isotherm (sample 4). After being ground and polished following standard procedures the 
samples were etched with hot picric acid solution. The etching procedure is :

1. Prepare a saturated solution by dissolving $5 \mathrm{~g}$ of picric acid in $100 \mathrm{ml}$ of $\mathrm{H}_{2} \mathrm{O}$. Add a few drops of liquid soap to improve wettability.

2. Dip fine polished sample maintaining the solution between 70 and $90{ }^{\circ} \mathrm{C}$ using a water bath.

3. Etch for 1 to 2 minutes, then slightly polish using fine alumina.

4. Repeat steps 2 and 3 until an adequate structure is obtained.

Samples at the ingot surface were cut from ingots XI and XII (after stirring was started). The area perpendicular to the ingot surface was polished and etched with hot picric acid.

Longitudinal samples of ingots XI and XII taken in the vicinity of the pool profiles marked with tungsten powder were prepared (polished up to $1 \mu \mathrm{m}$ diamond paste) for quantitative microanalysis. The samples were analysed for $\mathrm{Ni}, \mathrm{W}$ and $\mathrm{Cr}$ using WDX in the microspec WDX-2A. A line scan was performed for points located $1 \mathrm{~mm}$ apart by moving the stage manually to every point position. The area used for acquisition was an square of approximately $150 \mu \mathrm{m}$ per side. Because WDX analysis requires the stage to be tilted 45 degrees the samples were positioned in such a way that the scan direction was parallel to the stage $\mathrm{x}$-direction. The standards used were pure elements; details of their count acquisition are presented in table 3.5. The filament current was $4.0 \mathrm{nA}$. In order to take into account possible interferences from the matrix (mostly $\mathrm{Fe}$ ) the raw intensities were input to a Kevex 8000 version of the MAGIC IV program. 
Table 3.2: Experimental programme

\begin{tabular}{|c|c|c|l|}
\hline Run & $\begin{array}{c}\text { Electrode } \\
\text { material }\end{array}$ & $\begin{array}{c}\text { Exciting } \\
\text { current, A }\end{array}$ & Comments \\
\hline \hline I & 4340 & 380 & $\begin{array}{l}\text { Slag flow visualizaton and general ob- } \\
\text { servations }\end{array}$ \\
\hline \hline II & 4340 & 500 & Unsteady-state stirring \\
\hline III & 4340 & 350 & Unsteady-state stirring \\
\hline IV & 4340 & 100 & Unsteady-state stirring \\
\hline \hline V & 4340 & 200 & Steady-state stirring \\
\hline VI & 4340 & 350 & Steady-state stirring \\
\hline \hline VII & 4340 & 250 & Unsteady- and steady-state stirring \\
\hline \hline VIII & Cu-50 Fe & 350 & Unsteady-state stirring \\
\hline IX & Inconel 718 & 380 & $\begin{array}{l}\text { Effect of slag composition. Unsteady- } \\
\text { state stirring }\end{array}$ \\
\hline X & Inconel 718 & 380 & $\begin{array}{l}\text { Effect of slag composition. Unsteady- } \\
\text { state stirring. }\end{array}$ \\
\hline \hline XI & 4340 & 0 & Mixing (non-stirred) \\
\hline XII & 4340 & 380 & Mixing (stirred) \\
\hline \hline
\end{tabular}


Table 3.3: Chemical composition of slags used in the experimental programme

\begin{tabular}{|c|c|c|c|c|}
\hline \multirow{2}{*}{ Run } & \multicolumn{4}{|c|}{ Slag composition (\% by weight) } \\
\cline { 2 - 5 } & $\mathrm{CaF}_{2}$ & $\mathrm{CaO}$ & $\mathrm{Al}_{2} \mathrm{O}_{3}$ & $\mathrm{MgO}$ \\
\hline \hline I - VIII & 56.3 & 15.5 & 28.2 & - \\
\hline IX & 70.0 & 12.5 & 12.5 & 5.0 \\
\hline $\mathrm{X}$ & 40.0 & 25.0 & 25.0 & 10.0 \\
\hline XI - XII & 56.3 & 15.5 & 28.2 & - \\
\hline
\end{tabular}


Table 3.4: Nominal chemical composition of 4340 alloy steel, in weight percent.

\begin{tabular}{|cc|}
\hline$\% \mathrm{C}$ & $0.36-0.44$ \\
$\% \mathrm{Mn}$ & $0.55-0.80$ \\
$\% \mathrm{Cr}$ & $0.60-0.90$ \\
$\% \mathrm{Mo}$ & $0.20-0.30$ \\
$\% \mathrm{Ni}$ & $1.65-2.00$ \\
$\% \mathrm{P}$ & $<0.035$ \\
$\% \mathrm{~S}$ & $<0.400$ \\
$\% \mathrm{Si}$ & $0.15-0.30$ \\
\hline
\end{tabular}


Table 3.5: Count acquisition for the standards used in the WDX analysis.

\begin{tabular}{|c|c|c|c|c|c|c|c|c|}
\hline \multicolumn{9}{|c|}{$\begin{array}{l}\text { STANDARDS } \\
\text { SEM Beam voltage }=20 \mathrm{kV} \\
\text { Stage tilt }=45 \text { degrees } \\
\text { Take off angle }=45 \text { degrees }\end{array}$} \\
\hline \multirow[b]{2}{*}{ Z } & \multirow[b]{2}{*}{ Elem } & \multirow[b]{2}{*}{ Line } & \multicolumn{6}{|c|}{ Standard } \\
\hline & & & $\begin{array}{c}\mathrm{Abs} \\
\mathrm{Cur} \\
(n \mathrm{~A})\end{array}$ & $\begin{array}{c}\text { Peak } \\
\text { Counts }\end{array}$ & $\begin{array}{l}\mathrm{PkCt} \\
\text { Time } \\
(\mathrm{sec})\end{array}$ & $\begin{array}{l}\text { Bkgd } \\
\text { Counts }\end{array}$ & $\begin{array}{l}\mathrm{BkCt} \\
\text { Time } \\
\text { (sec) }\end{array}$ & $\begin{array}{l}\text { Counts } \\
\text { Per sec }\end{array}$ \\
\hline 24 & $\mathrm{Cr}$ & $\overline{K_{\alpha}}$ & 4.0 & 16564 & 10 & 12 & 5 & 1654.0 \\
\hline 28 & $\mathrm{Ni}$ & $K_{\alpha}$ & 4.0 & 15342 & 10 & 26 & 5 & 1529.0 \\
\hline 74 & W & $L_{\alpha_{1}}$ & 4.0 & 7888 & 10 & 83 & 5 & 772.2 \\
\hline
\end{tabular}




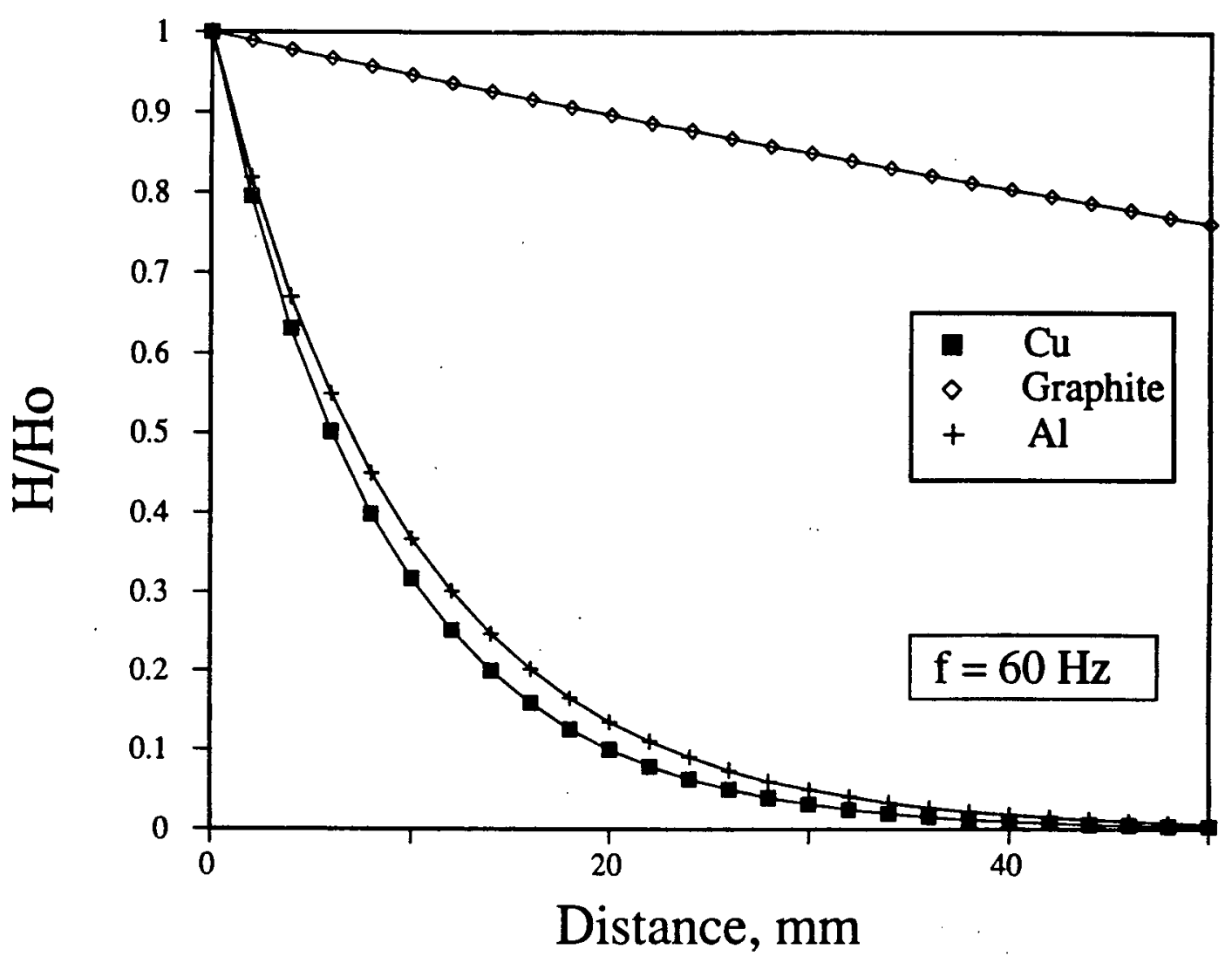

Figure 3.13: Magnetic field damping caused by the magnetic skin effect. 


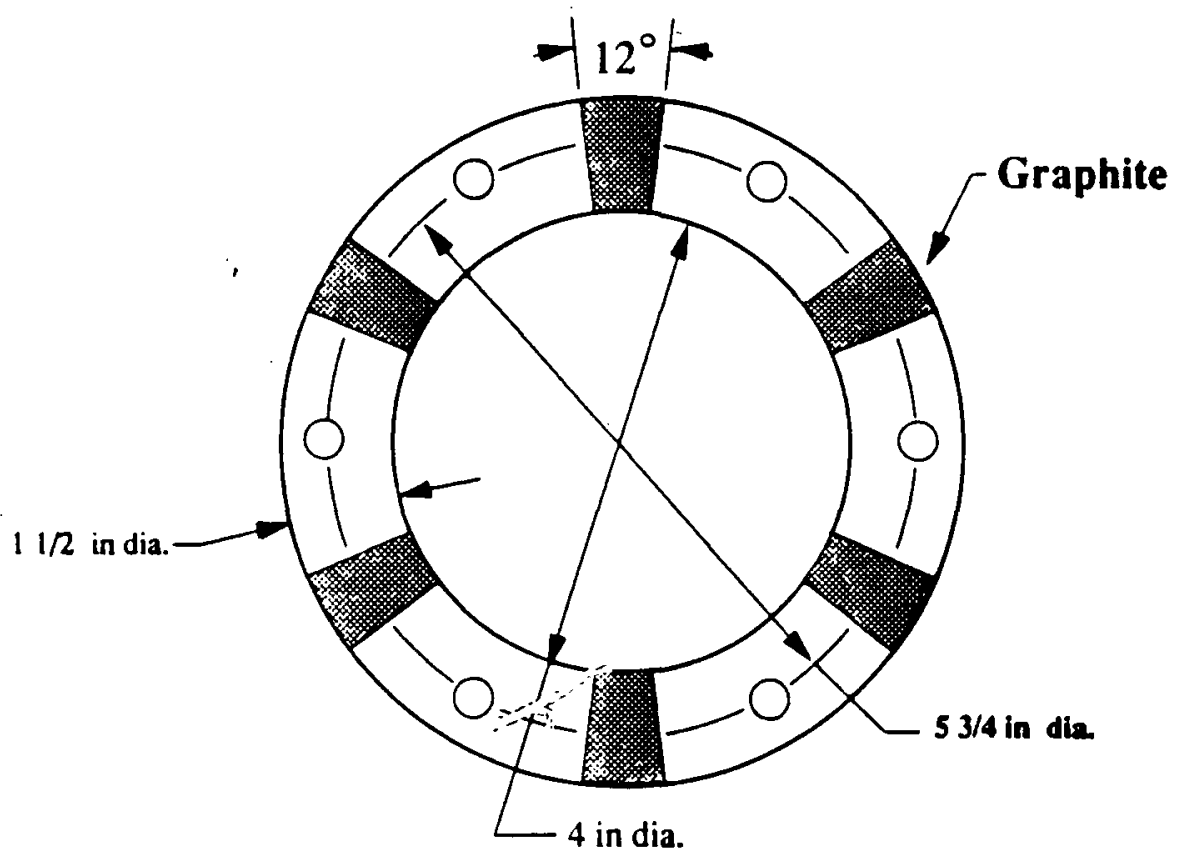

(a)

\section{Lateral view}

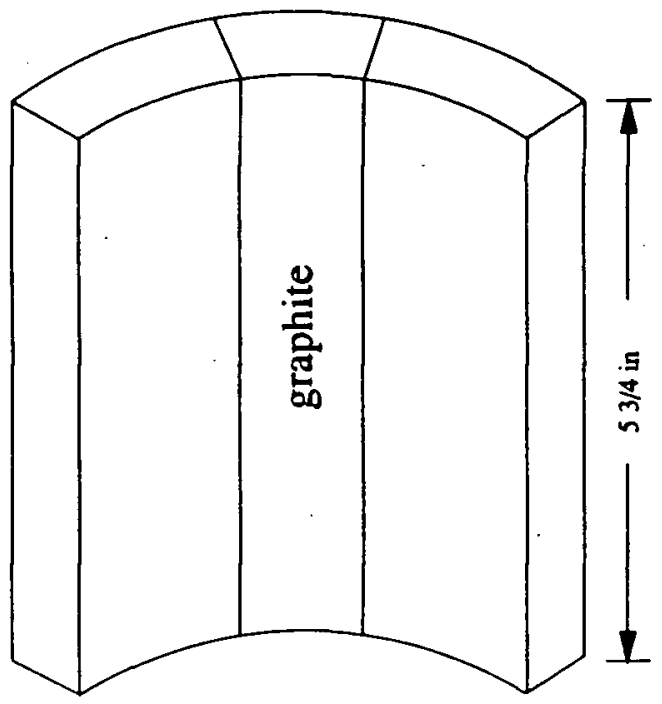

(b)

Figure 3.14: Schematic view of the stirring mould. (a) Top view. (b) Lateral view. 

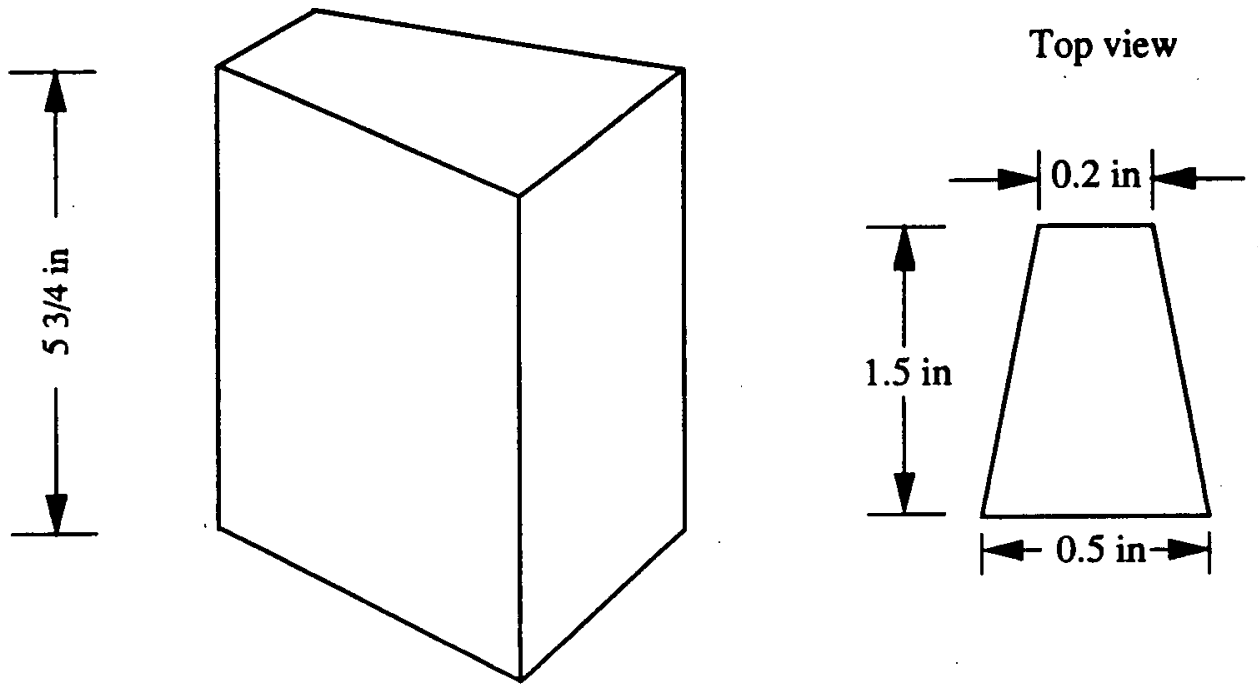

Figure 3.15: Graphite pieces used in the stirring mould. 


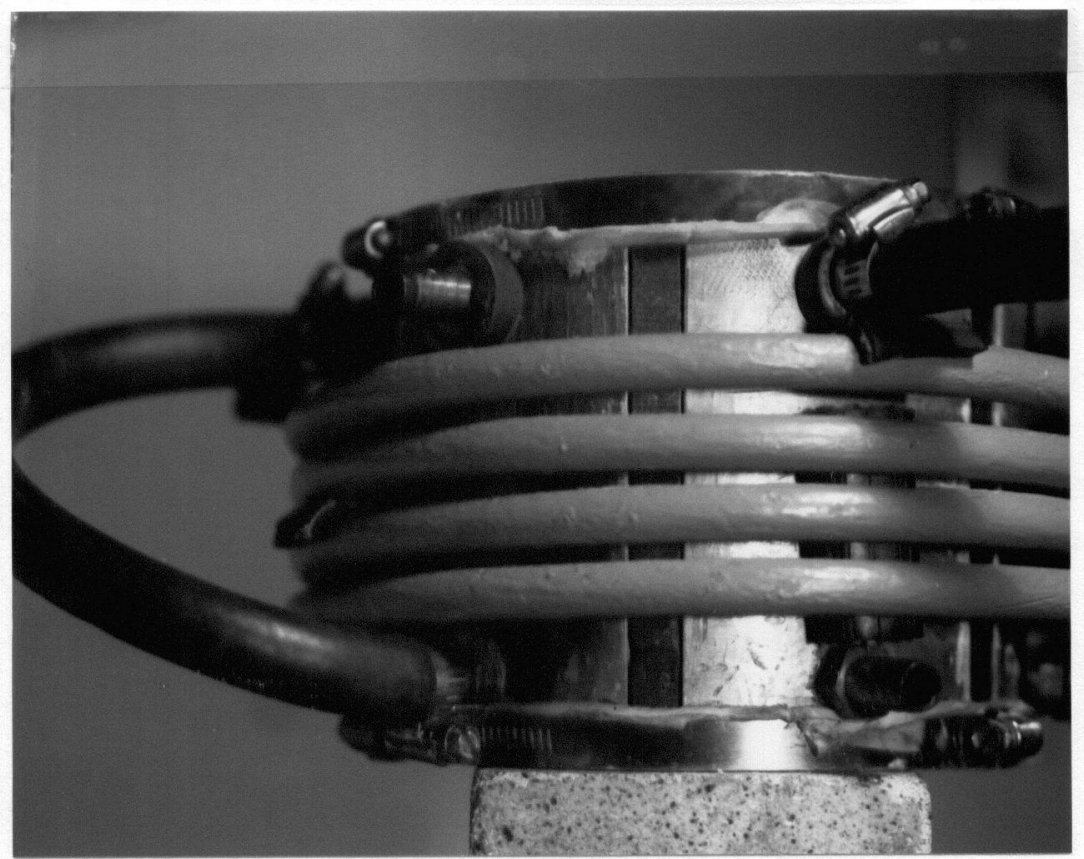

Figure 3.16: Detail of the stirring mould. 


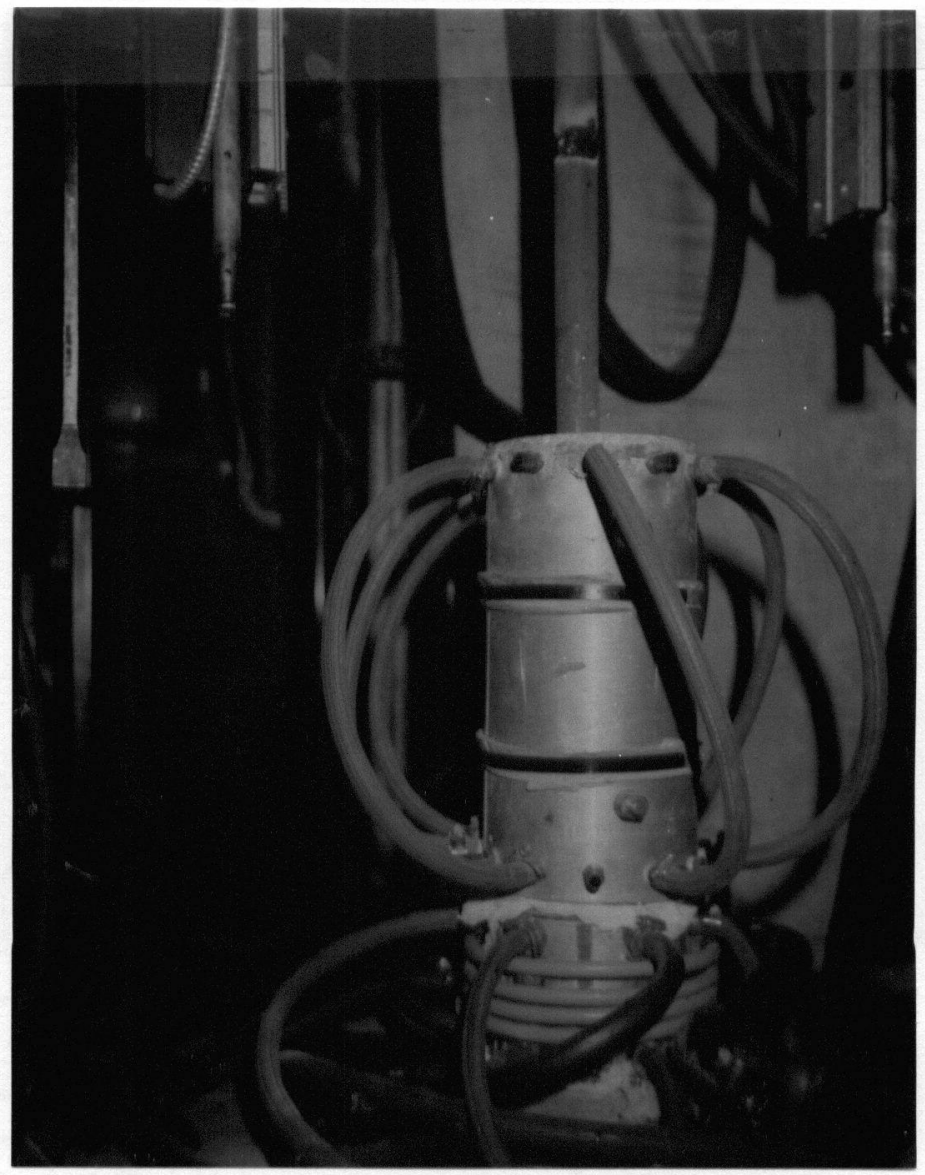

Figure 3.17: Mould arrangment used in the experimental programme. 


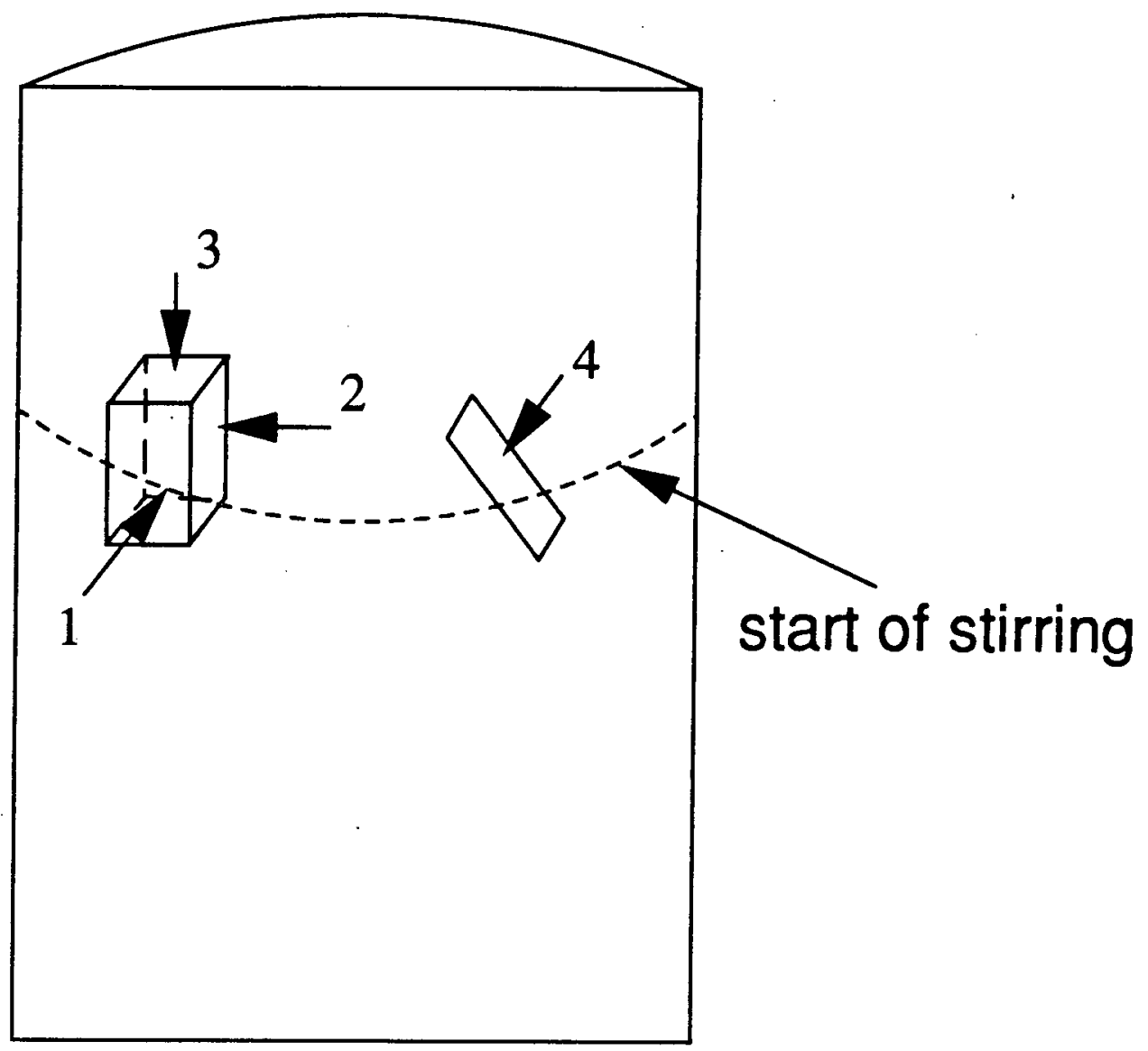

Figure 3.18: Samples from ingot VII. 


\section{Chapter 4}

\section{Results and Discussion}

\subsection{Magnetohydrodynamic Calculations}

The mathematical model developed to calculate the electromagnetic body force was used to simulate several induction arrangments, namely, frequency and magnitude of the exciting current, and number of turns and coil position with respect to the slag-metal interphase. The first part of the model simulates the magnetic field induced by the coil. This result is then used, together with a predefined current density distribution, to calculate the instantaneous magnetic body force.

\subsubsection{Electromagnetic Field}

The geometric parameters as well as the electrical properties of the several phases involved used in the simulations are summarized in table 4.6. The magnetic field density distribution obtained for those conditions is shown in fig. 4.19. The difference in electrical resistivity between the two phases is clearly reflected in the path of the magnetic field lines : for the liquid metal, most of the field is confined to the vicinity of the mould walls whereas it is more evenly distributed in the slag bath. It can also be observed that the flow lines diverge considerably at the interphase slag-metal pool, due to the presence of the magnetic skin.

The effect of varying the current passing trough the coil was investigated for frequencies of 20 and $1000 \mathrm{~Hz}$. The resulting magnetic field distributions are shown in figs. 4.20 
and 4.21, respectively. It can be noticed that a reduction in the frequency from 60 to $20 \mathrm{~Hz}$ does not alter significantly the magnetic field pattern, while a very high frequency $(1000 \mathrm{~Hz})$ produces a dramatic change. Since the magnetic skin varies inversely proportional to the square root of the frequency, at very high frequencies the depth of this conducting layer becomes extremely small.

The proportionality between the exciting current and the magnetic field is illustrated in figs. 4.22 and 4.23. The intensity of the field increased by a factor of 2 when the induction current was doubled. Similarly, the magnetic field density decreased by a factor of 10 when the exciting current was decreased to a tenth of its original value.

Coil geometry, i.e., number of turns and position is a very important parameter in processes where the fluids are at least partly driven by electromagnetic forces. Magnetic field patterns obtained when only one or two coils were used are shown in figs. 4.24 and 4.25. When these figures are compared with fig. 4.19 it is evident that the magnitude of the field increases with the number of coils. It should be noticed that such increment is much more pronounced in the vicinity of the mould wall (for the metal pool) and at the slag-metal interphase. It is very important to note that the amp-turn/m were kept constant in all the three cases which, according to the classical solution for an infinitely long solenoid fed with DC, should produce the same field intensity.

The effect of changing the position of the inductor with respect to the slag-metal. interface is illustrated in figs. 4.26 to 4.28 . The differences arising from the different positioning of the inductor result from the end effects associated with the fact that the coil has finite dimensions. Again, the changes are more noticeable inside the magnetic skin layers. 


\subsubsection{Electromagnetic Body Forces}

Once the induced magnetic field is calculated the magnitude of the electromagnetic body force in the regions of interest can be estimated from the cross product of that field and the current density. Since this force would only modify the hydrodynamic behaviour of the liquid phases a provision was made to reflect the solidified ingot.

As mentioned earlier, the current density distribution in the slag-metal system obtained by Jardy et al. [132] was used as a complementary input to the model. Such a distribution is shown in fig. 4.29 from where it can be noticed that the current density vectors at the lateral surface of the charge are parallel to the mould wall. This kind of distribution arises from the assumption that the mould does not carry any current at all. Also, there are two definite zones where the current diverges : 1) at the slag-argon interphase, because of the change in cross section, and 2) at the slag-liquid metal interphase, due to the magnetic skin effect (the electrical resistivity of the liquid metal being much smaller than that of the molten slag).

Results for the $\theta$-component of the instantaneous electromagnetic body force were obtained for the same conditions used in the previous subsection. It should be pointed out that the magnitude of this body force is influenced by the phase angle between the magnetic field and the electric current. For these simulations they were assumed to be in phase. Additionally, one is more concerned with the time averaged value of this body force and, therefore, conclusions drawn from the interaction of a magnetic field varying with time at a different frequency than the one of the current are meaningless.

The magnetic body force generated when the current density distribution shown in fig. 4.29 interacts with the magnetic field illustrated in fig. 4.19 is shown in fig. 4.30. The main feature of this particular plot is the fact that the magnetic body force is larger in the metal pool than in the slag. Since this body force accelerates the fluid it follows that 
a larger increment in velocity should be expected in the liquid pool when the external magnetic field is applied. It is interesting to note that a theoretical analysis for the stirring of an ESR furnace operated with DC using a DC magnetic field rotating in the azimuthal direction [117] predicted the same kind of behaviour.

Results for different exciting current values as well as number of coils and inductor position were also obtained. Since there was only a single current density distribution used for producing all these results, the conclusions that can be drawn are similar to the ones already discussed in the previous subsection and, therefore, will not be considered here.

A calculation of the electromagnetic body force distribution for a $500 \mathrm{~mm}$ dia. ingot produced with an operational current of $10 \mathrm{kA}$ was also performed. The resulting distribution is shown in fig. 4.31. Again, most of the stirring would be confined to the magnetic skin layers.

With the electromagnetic body force distribution already calculated one might be tempted to calculate the velocity distribution. Unfortunately, such a calculation requires the full solution of the Navier-Stokes equation as demonstrated by [137].

\subsection{Electromagnetic Stirring During ESR}

\subsubsection{General Observations}

The high temperatures involved in the ESR process as well as the chemical aggressiveness of the slag make it difficult to carry out measurements of velocity profiles in the system. Consequently, the presence of the externally applied magnetic field has to be assessed through some indirect observations.

For one experiment (run I) the insulating material normally placed at the top of the mould arrangment was removed and it was possible to visually inspect the slag surface 
before and after the start of EMS in runI. During the normal operation the slag was slowly rotating clockwise; after stirring was started, the surface of the slag rotated vigorously in the same direction at an estimated angular velocity of $1 \mathrm{rev} / \mathrm{s}$. As a consequence of this rotation a deep vortex was created around the electrode. Virtually no time delay was observed between the start of EMS and the increase in rotational velocity in the slag. In contrast, some time was required after the stirring device was switched off before the slag recovered its original degree of motion, this being a result of the momentum generated by the stirring. Another evidence of the increased fluid motion caused by stirring is the presence of surface ripples around the diameter of the ingot when high exciting current levels were used. A detail of the surface of ingot II at a position where stirring had just started is shown in fig. 4.32. The rapid increase in the rotational speed of the slag bath generated by EMS can be postulated as responsible for the formation of such a gap between the mould and the slag skin.

The melting current flowing through the electrode decayed noticeably when the stirrer was switched on. It is thought that this behaviour results from the vortex created in the slag bath which may, for large values of the exciting current, cause a decrease in electrical potential because the electrode loses contact with the slag and therfore the circuit cannot be closed. In order to keep a constant current flowing through the system the rate of electrode travel has to be increased and this is reflected when the melting rate is plotted against time (see fig. 4.33). It is then apparent that the 'increase' in the melting rate is not a direct result of EMS but rather a consequence of the electrical requirements of the installation which are perturbed by the stirring. Several research efforts $[113,116]$ have pointed out an increase in the melting rate resulting from EMS; however they do not report any adjustment required in the electrode rate of travel. Regarding the melting rate calculation it has to be recalled that the ingot rises in an opposite direction to the electrode travel and therefore both, ingot and electrode velocity, have to be taken into 
account. A mass balance that includes such a correction gives the melting rate as a function of electrode speed and electrode and ingot areas :

$$
\mathrm{MR}=\frac{\pi}{4} D_{i}^{2} \rho_{i} u_{e}\left(\frac{D_{e}^{2}}{D_{i}^{2}-D_{e}^{2}}\right)
$$

Additionally, the ingot cross-sectional area varies from mould to mould in the experimental setup. Consequently, the melting rate was calculated only for the stirring section of the mould when fig. 4.33 was generated.

The energy efficiency of the furnace is plotted as a function of time in fig. 4.34. If one postulates that the stirring will cause more heat to be dissipated towards the mould wall the energy efficiency of the furnace should be diminished. That is exactly the trend shown in that plot.

\subsubsection{Metallurgical Consequences of EMS}

\subsubsection{Unsteady-state Stirring}

Photomacrographs of ingots II to IV are shown in figs. 4.35 to 4.37 corresponding to exciting currents of 500,350 and $100 \mathrm{~A}$, respectively. From these phothographs it is clear that the stirring will have a more pronounced effect as the current increases. They also provide a reference for the curent level required to achieve a minimum effect in the macrostucture, as ingot IV hardly shows any difference from a conventionally remelted ingot.

The fluid flow perturbation originated by the additional convective component generated by the magnetic field results in a region showing a different structure where the start and end of stirring are clearly discernible. From the photographs one can observe that this region is larger at the ingot center and practically non existent towards the surface. This behaviour can be explained considering that the electromagnetic body force acts on 
a volume of fluid and that there is more volume at the center of the ingot (larger mushy zone). A detail of a stirred zone in ingot III (taken without further metallographic work) is shown in fig. 4.38. Two lines can be clearly seen at the bottom of the picture. It is reasonable to suggest that the first one results from the abrupt change in fluid flow conditions while the second arises from increasing the electrode rate of travel. The strongly directional structure present before EMS was started continues for a definite distance after which shorter, randomly oriented dendrites can be observed. This particular structure is known as columnar branched. The upper boundary of the stirring zone is also clearly revealed by the etch. The survival of the original structure well into the stirred zone suggests that the growing dendritic front does not immediately 'see' the change in thermal conditions consequence of the stirring, presumably because the fluid flow in the fully liquid zone ahead of the tip of the dendrites does not penetrate deep enough into the interdendritic zone to cause a drastic change in the solidification conditions, although it is enough to modify the segregation patterns as revealed by the macroetch.

It has been shown that the effect of forced convection on macrosegregation patterns is affected by the thermal gradient at the solidification front and the solidification rate, as well as the length of the mushy zone. Predicted values of these parameters are shown in fig. 4.39 for this particular ESR installation. In general, these conditions favour the directional structures characteristic of ESR ingots.

A photograph of the microstructure of an stirred zone of ingot II, as revealed by Oberhoffer's reagent, is presented in fig. 4.40. In this photograph several macrosegregated zones can be observed, mainly at both ends of the stirring zone. They resemble the 'semimacroscopic' centreline segregation found in slabs produced by continuous casting with EMS [138]. These macrosegregated areas were qualitatively analyzed for $\mathrm{P}$ and $\mathrm{C}$ using SEM-WDX. An SEM picture, along with the WDX line scans, are shown in fig. 4.41. From this figure it can be concluded that the segregated zones are rich in P. A sample 
cut in a perpendicular direction was metallographically prepared in the same way and the resulting structure is shown in fig. 4.42. A larger occurrence of such segregated areas can be seen in this picture but no evidence of channels can be found. Therefore, it can be postulated that they are formed by solute rich interdendritic liquid trapped in the randomly oriented structure produced by the stirring.

\subsubsection{Steady-state Stirring}

The photomacrographs of ingots V-VII are shown in figs. 4.43 to 4.45 . The lines shown in the pictures are liquidus isotherms as marked by tungsten powder additions.

In contrast to the macrostructures produced by unsteady-state stirring, no drastic changes are visible in this ingots. It is particularly interesting to compare figs. 4.36 and 4.44 which show the macrostructure obtained when an exciting current of $350 \mathrm{~A}$ is applied under unsteady- and steady-state conditions, respectively. The different shape of the pool profiles suggest that the thermal field cannot recover from the sudden increase in liquid motion in the short time during which the stirrer was on for the unsteady-state case. It is also interesting to note the asymmetric profiles produced by the stirring. Both, a change in the drop motion pattern through the slag [119] and electrode asymmetry [117] has been postulated as reasons for such a behaviour. In any case, this lack of symmetry suggests that the two-dimensional analysis of the thermal field generally used to model the conventional ESR may not be entirely adequate when the melt is stirred by means of an electromagnetic field.

\subsubsection{Effect of Pool Profile}

Since the electromagnetic body force acts on fluid volume elements, the pool depth and shape should play an important role in determining the liquid metal motion. For a given set of electrical conditions and mould geometry the shape of the pool profile will depend 
on the thermophysical properties of the remelted material (particularly, liquidus and solidus temperatures) as well as on the electrical resistivity of the slag. Therefore, runs VIII to $\mathrm{X}$ can be viewed as examples of the role played by the pool geometry in the overall liquid motion.

A photograph of ingot VIII $(\mathrm{Cu}-50 \% \mathrm{Fe})$ is shown in fig. 4.46. The susbstancial temperature difference between liquidus and solidus in this particular alloy $\left(\sim 340^{\circ} \mathrm{C}\right)$ is reflected in a large mushy zone. The porosity defects and large segregated areas observed in the photograph are a direct consequence of the larger volume of liquid metal present during solidification and the larger local solidification times.

Experiments IX and $\mathrm{X}$ were run under two different slag compositions. When the $\mathrm{CaF}_{2}$ content of the slag is decreased its electrical resistivity increases [139] which favours heat generation in the slag bath and, consequently, modifies the pool shape. Figs. 4.47 and 4.48 show the macrostructures of ingots IX and X, respectively. Clearly, the deeper pool profile of ingot IX favoured a stronger stirring of the liquid pool.

\subsubsection{Liquid Metal Flow}

The main objective of applying the external field is to increase the motion of the liquid metal. Experimentally, due to the non-transparent nature of liquid metals, it is not possible to visualize such a flow and one has to resort to changes produced in the solidified structure.

A microstructural feature commonly used to characterize the flow patterns present when stirring is applied during solidification is the deflection angle of the primary dendrites with respect to the growing direction at the solidification front $[140,141]$. Four samples ( fig. 3.18 ) where obtained at the point where stirring was started in run VII (steady-state stirring) and etched with hot picric acid in order to reveal the dendritic structure. Photomicrographs of the etched samples are shown in figs. 4.49 to 4.52 . The 
strongly columnar growth characteristic of ESR (as evidenced in fig. 4.51) was not significantly disturbed by the stirring device, the only possible evidence of an increase in the fluid flow being the thin white band present at the point where stirring was assumed to start. This band is similar to the 'white band' present when EMS is applied during continuous casting of steel (although in that case marks the boundary between columnar and equiaxed zones [142]) and can be attributed to liquid suddenly sweeping the upper boundary of the mushy zone.

In order to complement these observations, samples were taken from ingotsXI andXII. The microstructures are shown in figs. 4.53 and 4.54 , which correspond to cross-sectional samples in the vicinity of the ingot surface for non-stirred and stirred sections. Because of the liquid head at the top of the ingot and the highly directional heat transfer at the mould wall, fig. 4.53 shows a high degree of directionality in the array of dendrites. On the other hand, the flow induced by the stirrer completely destroyed this directionality, as shown in fig. 4.54. This result seems to contradict the observations obtained from the experiments involving unsteady-state stirring; where no appreciable change in structure was observed at the ingot surface. The difference arises from the fact that fig. 4.54 shows a zone where dendrites are just starting to grow and therefore the disturbance at the solidification front caused by the stirring modifies the conditions under which these dendrites grow.

\subsection{Mixing in the Liquid Pool}

The mixing behaviour of continuous flow systems is influenced by the distribution of residence times of the various fluid elements. It is common practice to estimate this distribution by adding a definite amount of a chemical or radioactive tracer to the input stream and then following its behaviour as it exits the reactor [143]. The tracer can be 
added either continuously during a long period of time (step input) or in a pulse. In this work, nickel powder was added in a pulse at a given time after stirring was started. The non-stirred case was also investigated. In an ESR operation, the liquid pool acts as the 'vessel' and the tracer behaviour is monitored via chemical analysis of longitudinal samples. Along with nickel, tungsten powder was added to identify the point of addition. The chemical analysis was performed using SEM-WDX. The ZAF corrections for the analysis were generally small (the difference between $K$ ratio and weight fraction being in the order of $5 \%$ for $\mathrm{Cr}$ and $\mathrm{Ni}$, and $10 \%$ for $\mathrm{W}$ ).

The results for stirred (run XI) and non-stirred (run XII) ingots are shown in figs. 4.55 and 4.56. In the stirred case the nickel profile suggests that most of the powder was dissolved and then uniformly distributed by the complex fluid flow pattern created by the additional stirring. In contrast, the high peak in the nickel concentration observed in the non-stirred experiment reveals that the powder went through the liquid pool and up to the dendrite tips where it was trapped by the growing dendritic array. Since no periodic variations in the concentration were observed it can be assumed that the liquid metal motion does not follow a simple recirculating pattern.

This behaviour was quantified via both the calculation of the effective distribution coefficient for $\mathrm{Ni}\left(k_{\mathrm{eff}}^{*}\right)$ and the reactor dispersion number (inverse of the axial Peclet number).

For the effective distribution coefficient calculations the decay part of the tracer distribution curves was analyzed following the method proposed by Mitchell et al. [99]. The value of $k_{\mathrm{eff}}^{*}$ is obtained from fitting the experimental curve to the following equation : .

$$
C_{s_{n}}=\left(C_{l}-C_{s} \frac{\Delta h}{h_{o}}\right)_{n-1}
$$

where $\Delta h$ is the volume increment on an ingot of constant liquid pool $h_{o}$. The value of $h_{\circ}$ was estimated from the pool profiles as marked by tungsten powder. 
The best fit values for the experiments with and without stirring are shown in fig. 4.57. Since $1<k_{\mathrm{eff}}^{*}<k_{o}$ (where $k_{o}$ is 0.39 as calculated from the Fe-C diagram for $0.4 \mathrm{w} / \mathrm{o} \mathrm{Ni}$ ) some degree of mixing exists inside the liquid pool. The smaller value of $k_{\text {eff }}^{*}$ corresponding to the experiment with stirring proves that a higher degree of mixing is present in the liquid for this case.

The classical dispersion number analysis was also used. Because of its higher accuracy the $\mathrm{C}$ curve representation was prefered over the $\mathrm{F}$ diagram. The full set of experimental values was transformed according to :

$$
C=\frac{c}{w / V}
$$

where

$$
\begin{aligned}
c & =\text { exit concentration, } \mathrm{g} \mathrm{cm}^{-3} \\
w & =\text { quantity of tracer added, } \mathrm{g} \\
V & =\text { volume of fluid in the vessel, } \mathrm{cm}^{3} .
\end{aligned}
$$

and

$$
\begin{aligned}
& \theta=\frac{t}{\bar{t}} \\
& \bar{t}=\frac{V}{Q}
\end{aligned}
$$

where

$$
\begin{aligned}
\bar{t} & =\text { average residence time, } \mathrm{s} \\
Q & =\text { volumetric flow rate, } \mathrm{cm}^{3} \mathrm{~s}^{-1}
\end{aligned}
$$

Both, the volumetric flow rate and the transformation from ingot height to time were estimated from an early determined ingot growth velocity $\left(0.0158 \mathrm{~cm} \mathrm{~s}^{-1}\right)$.

The inverse of the axial Peclet number was obtained by fitting values to the theoretical curve represented by

$$
C=\frac{1}{2 \sqrt{\pi \theta P e^{-1}}} \exp \left(-\frac{(1-\theta)^{2}}{4 \pi P e^{-1}}\right)
$$


When $P e^{-1} \rightarrow 0$ plug flow (no mixing) predominates while $P e^{-1} \rightarrow \infty$ represents complete mixing. Since eq. 4.57 is derived for a vessel originally free of the addition, the theoretical $\mathrm{C}$ values where corrected for the nickel concentration in the original electrode. The resulting curves are shown in figs. 4.58 and 4.59. Once again the experiments with stirring are best described by a higher degree of mixing, $P e^{-1}$ of 0.14 and 0.025 for stirred and unstirred ingots, respectively.

\subsection{Thermal Regime}

The additional stirring created by the electromagnetic field modifies the way heat is transported during ESR and consequently alters the thermal field. Experimental evidence of such modification was found by inspecting the pool profile as well as the energy efficiency of the installation.

An existing computer code was modified and used to investigate the changes in the predicted pool profiles when heat transfer inside the liquid pool is altered. Since the boundaries of the system did not suffer any modification, the changes will only reflect variations in the convective transport of heat inside the pool. Early attempts to predict the exact geometry of the experimental runs failed to produce any results due to numerical instabilities, presumably related to the small ingot size. Consequently, an hypothetical ingot (30 in dia., 90 in long) had to be considered. The melting rate was assumed to be $750 \mathrm{~kg} / \mathrm{h}$. The original version of the code was used, first, to simulate the remelting of the three alloys used in the experimental programme. The pool profiles for 4340 allloy steel, $\mathrm{Cu}-50 \% \mathrm{Fe}$ and Inconel 718 are shown in figs. 4.60 to 4.62 , respectively.

For the rest of the simulations, the thermophysical properties of the 4340 steel were employed.

The vigorous rotation induced in the slag bath implies a more uniform temperature 
distribution across it. Since the mathematical model relates the thermal phenomena in the slag and metal through a temperature distribution at the top of the remelted ingot, several distributions were simulated (see fig. 4.63). The corresponding solidus and liquidus isotherms are shown in figs. 4.64 to 4.66 . In spite of the big differences among the temperature profiles used in this computations the resulting thermal field hardly shows any difference with respect to the reference ingot ( fig. 4.60 ).

The modifications done in the computer code allow the simulation of different levels of convective heat transfer inside the metal pool. Moreover, it is possible to have a larger amount of heat transported in either the radial or axial directions.

Figs. 4.67 and 4.68 illustrate the effect of an increase in the effective thermal conductivity in the liquid pool. In these cases both, radial and axial components of the flow were increased by the same factor. The general trend shows an increase in the pool depth as the convective heat transport is increased (larger values of $k_{\text {eff }}$ ).

Presumably, the effect of the external field will not be the same along the radius of the liquid melt. Therefore, several simulations were done where the effective thermal conductivity was a function of the radial position. Additionally, several values of the ratio axial to radial convective heat transport, $k_{\text {eff }}^{\mathrm{z}} / k_{\text {eff }}^{\mathrm{r}}$ were tested. Representative results of the thermal profiles obtained are shown in figs. 4.69 to 4.71 . In all the cases, an isotropic effective thermal conductivity was used for nodes 4 to 11 in the radial direction, while anisotropic values were chosen for the nodes close to the ingot axis.

Figs. 4.69 and 4.70 show the results of using a base effective thermal conductivity equivalent to $2 \times k_{\text {at }}$, modified at the central nodes for preferential transport in the axial or radial direction, respectively. It can be observed that the modifications implemented alter significantly the central region of the pool : if the heat is mostly transported in the axial direction, a flatter pool profile and an smaller mushy zone are obtained. The thermal field resulting when the base thermal conductivity is increased to $5 \times k_{\text {at }}$ is shown 
in fig. 4.71. For this run, the ratio $k_{\text {eff }}^{\mathrm{z}} / k_{\text {eff }}^{\mathrm{r}}$ was 0.2 in the central nodes. The pool shape is similar to the one of fig. 4.70 but the pool depth is larger.

Some of the experimentally obtained profiles (see fig. 4.45, for example) showed a particular shape that suggest that the heat is transported in a different way at the center of the melt. It was not possible to reproduce such a profile with the modified code. 
Table 4.6: Geometric parameters and electrical properties used in the magnetohydrodinamic calculations.

Crucible heght, crucible I.D., crucible thickness, $\mathrm{h}$, coil radius, number of turns, frequency, number of nodes : radial direction axial direction crucible electrical conductivity, slag electrical conductivity, melt electrical conductivity,
7.0 in 10.0 in

1.25 in

$0.0148 \mathrm{~m}$

7.5 in

4

$60 \mathrm{~Hz}$

16

12

$4 \times 10^{5}(\Omega m)^{-1}$

$4 \times 10^{2}(\Omega m)^{-1}$

$4 \times 10^{5}(\Omega m)^{-1}$ 


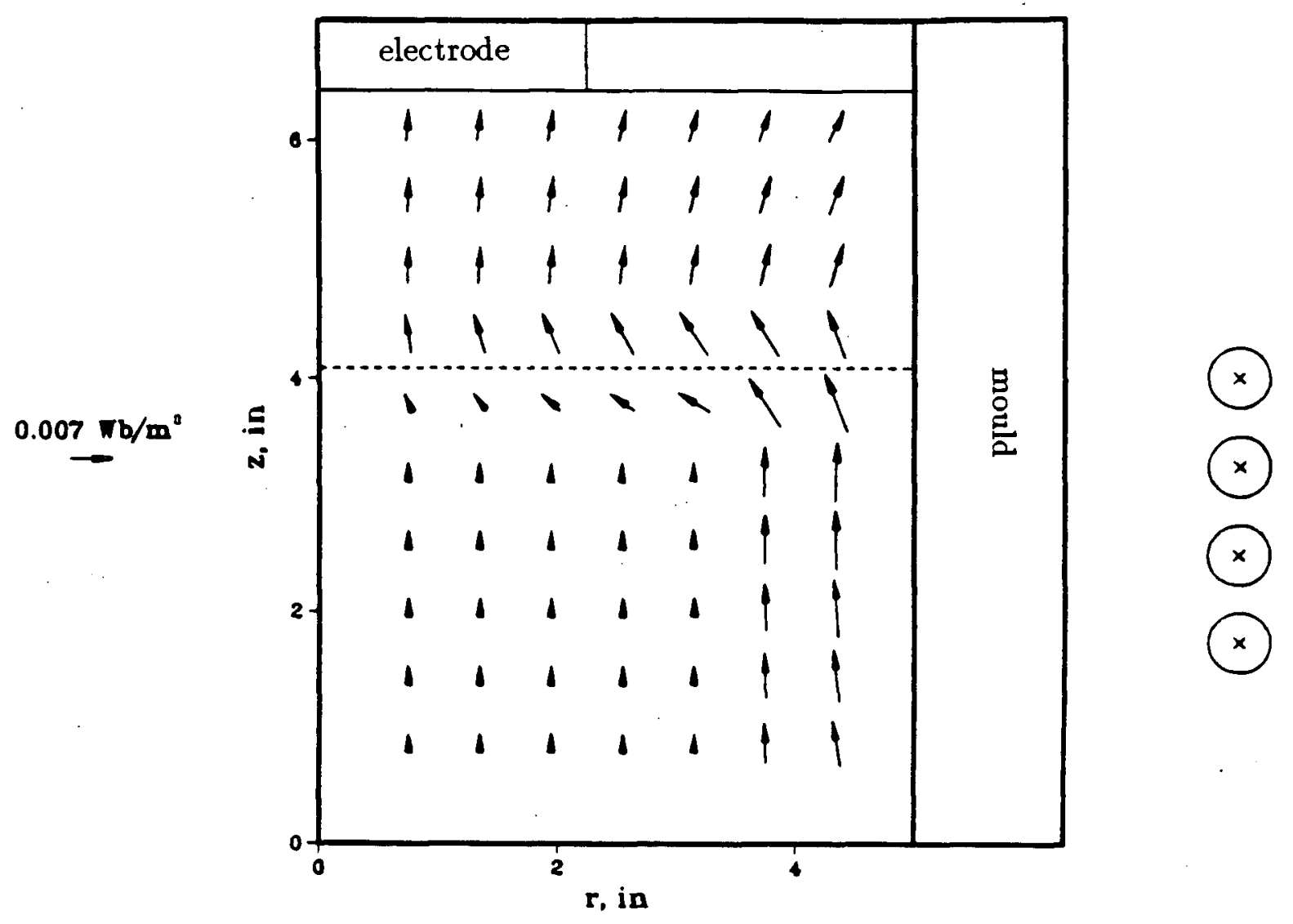

Figure 4.19: Magnetic field distribution obtained for the conditions shown in table 4.6. 


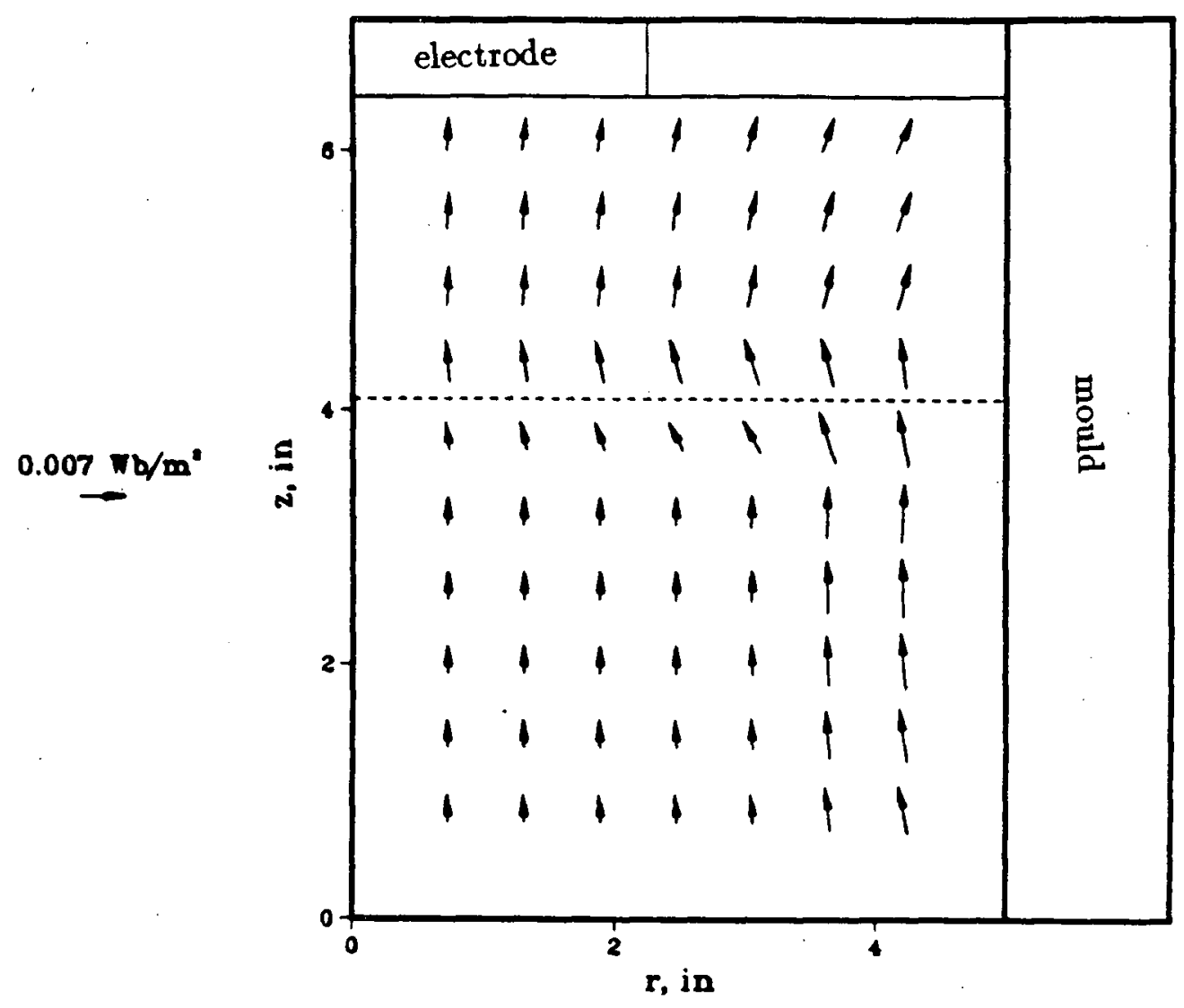

Figure 4.20: Magnetic field distribution obtained for $\omega=20 \mathrm{~Hz}$. 


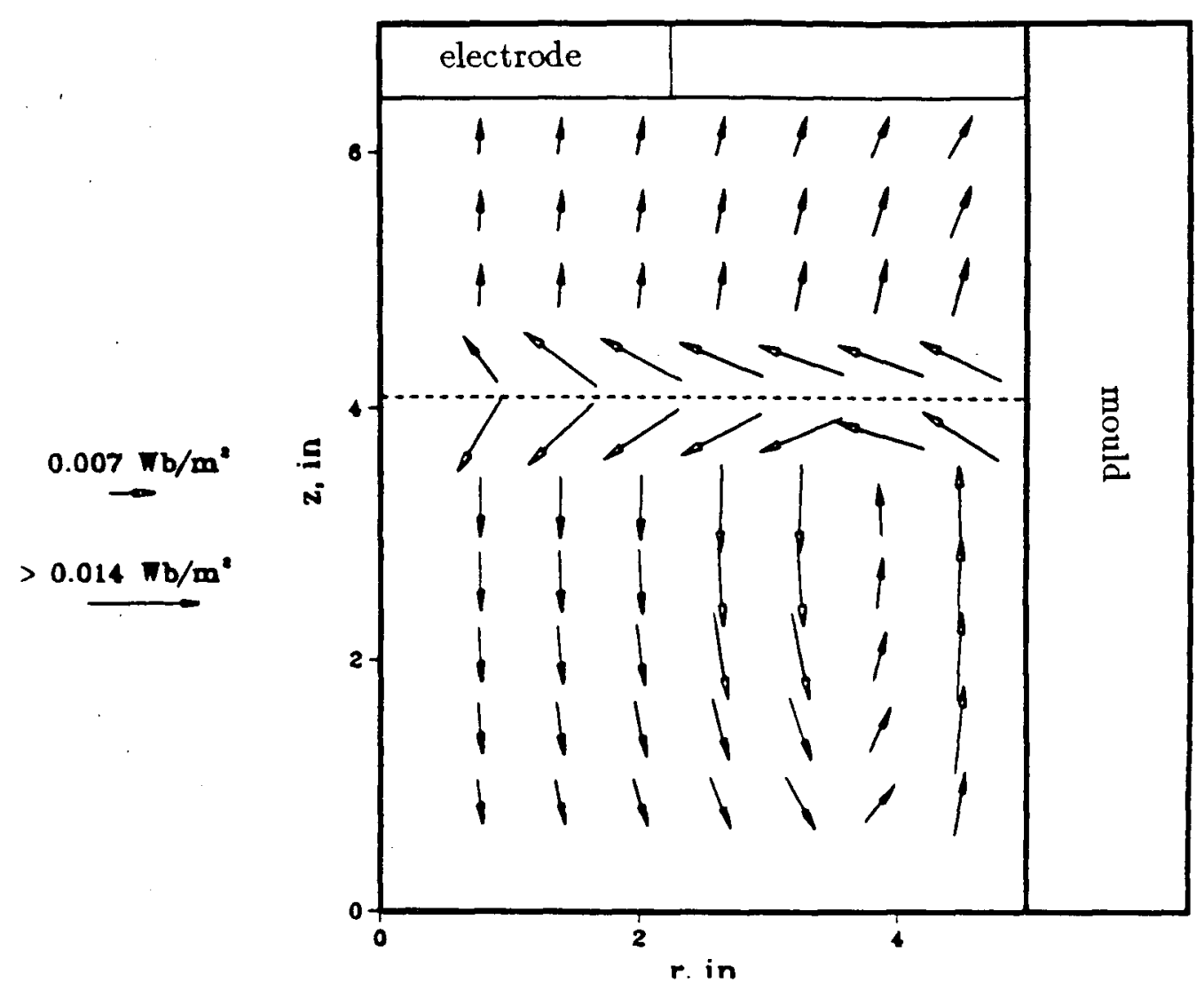

Figure 4.21: Magnetic field distribution obtained for $\omega=1000 \mathrm{~Hz}$. 


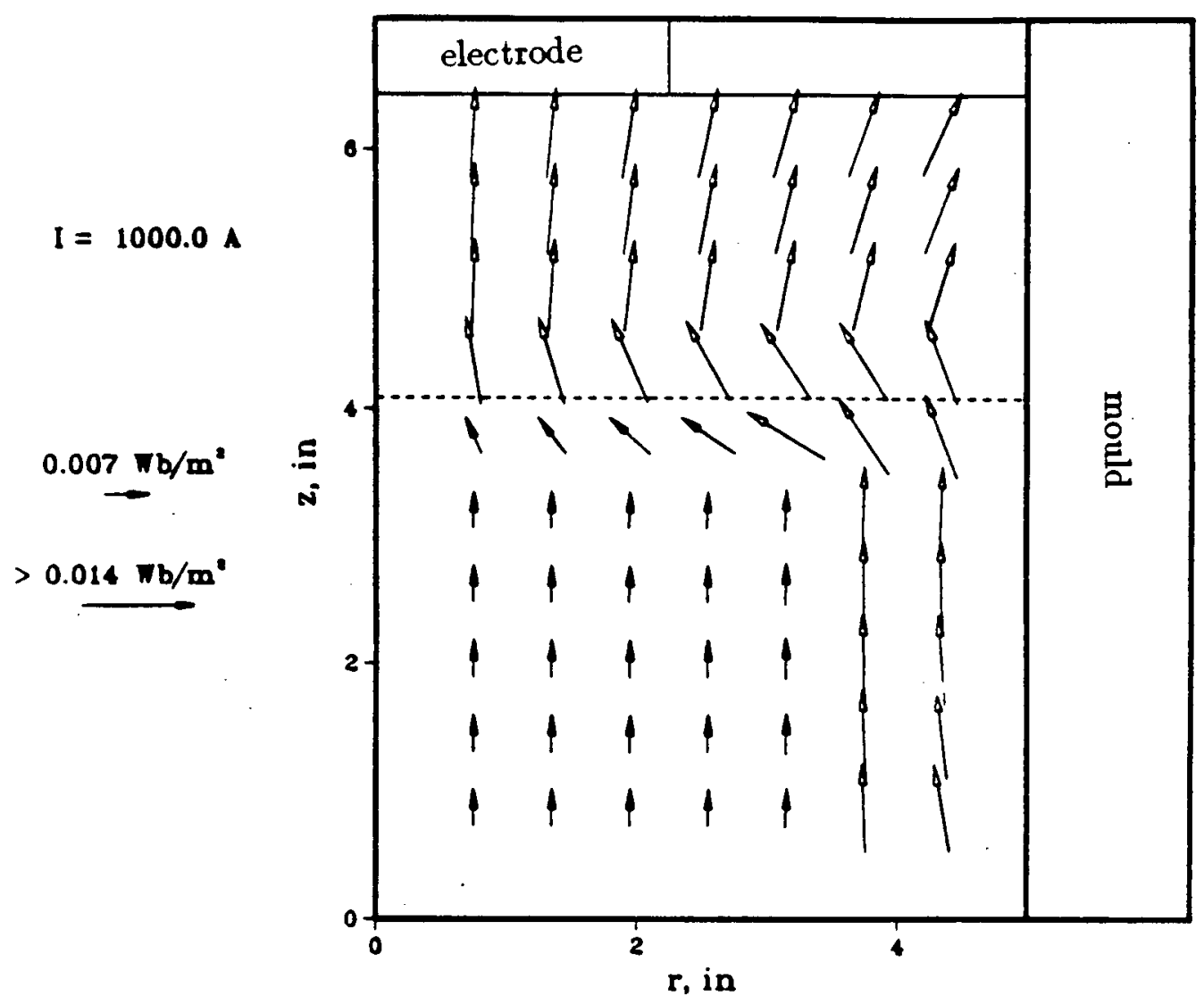

Figure 4.22: Magnetic field distribution obtained for an exciting current of $1000 \mathrm{~A}$. 


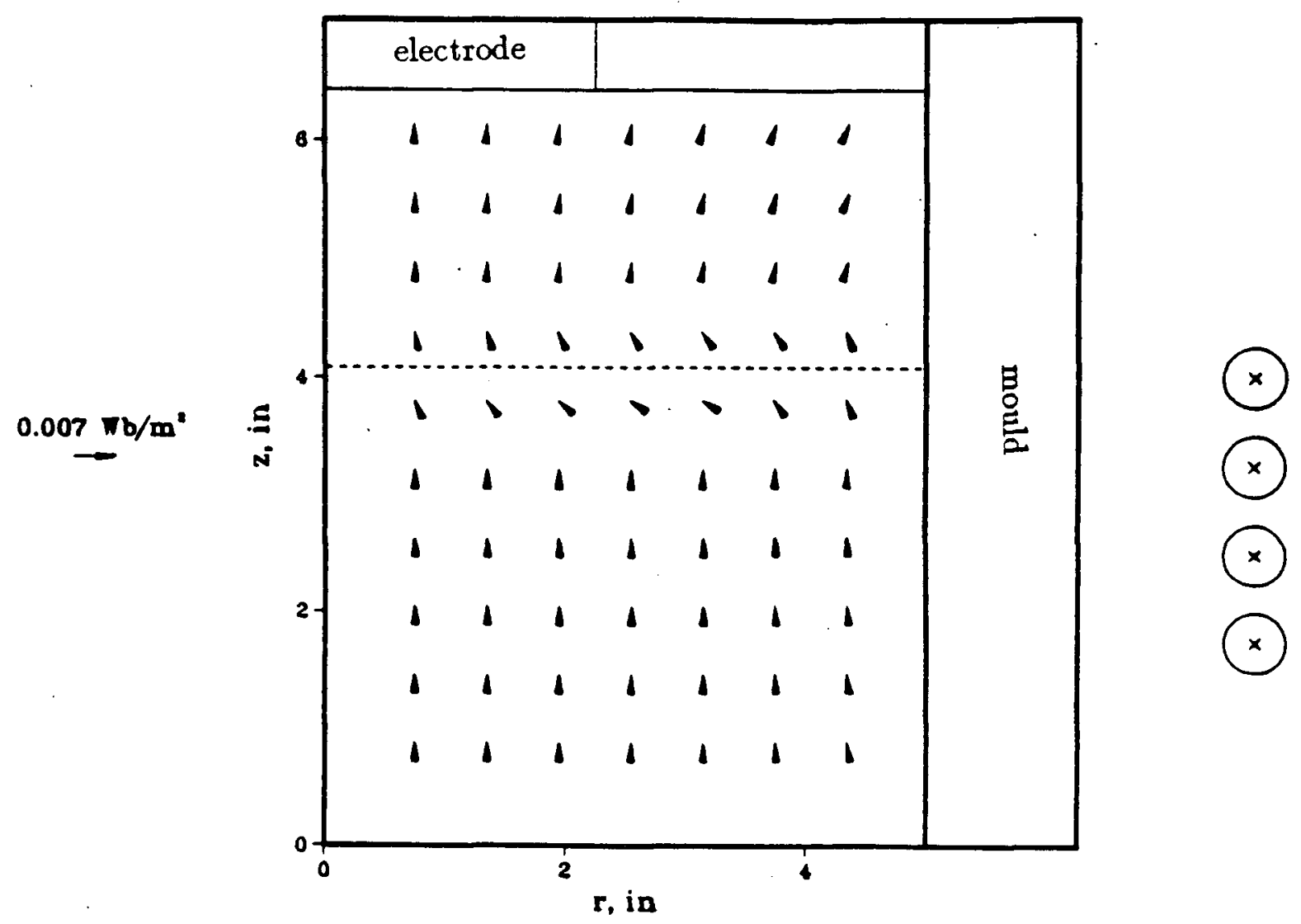

Figure 4.23: Magnetic field distribution obtained for an exciting current of $50 \mathrm{~A}$. 


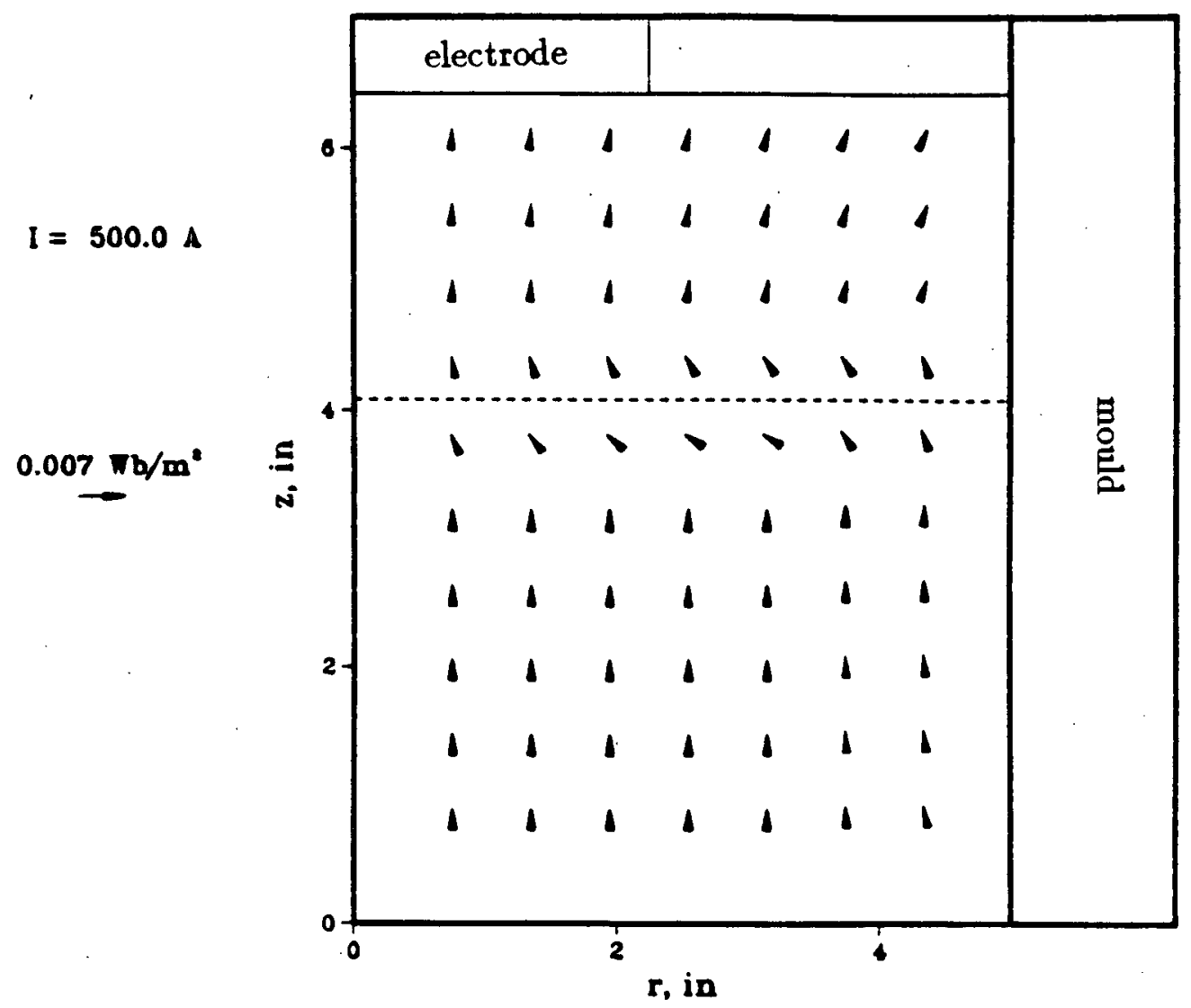

Figure 4.24: Magnetic field distribution obtained when the coil has only one turn. 


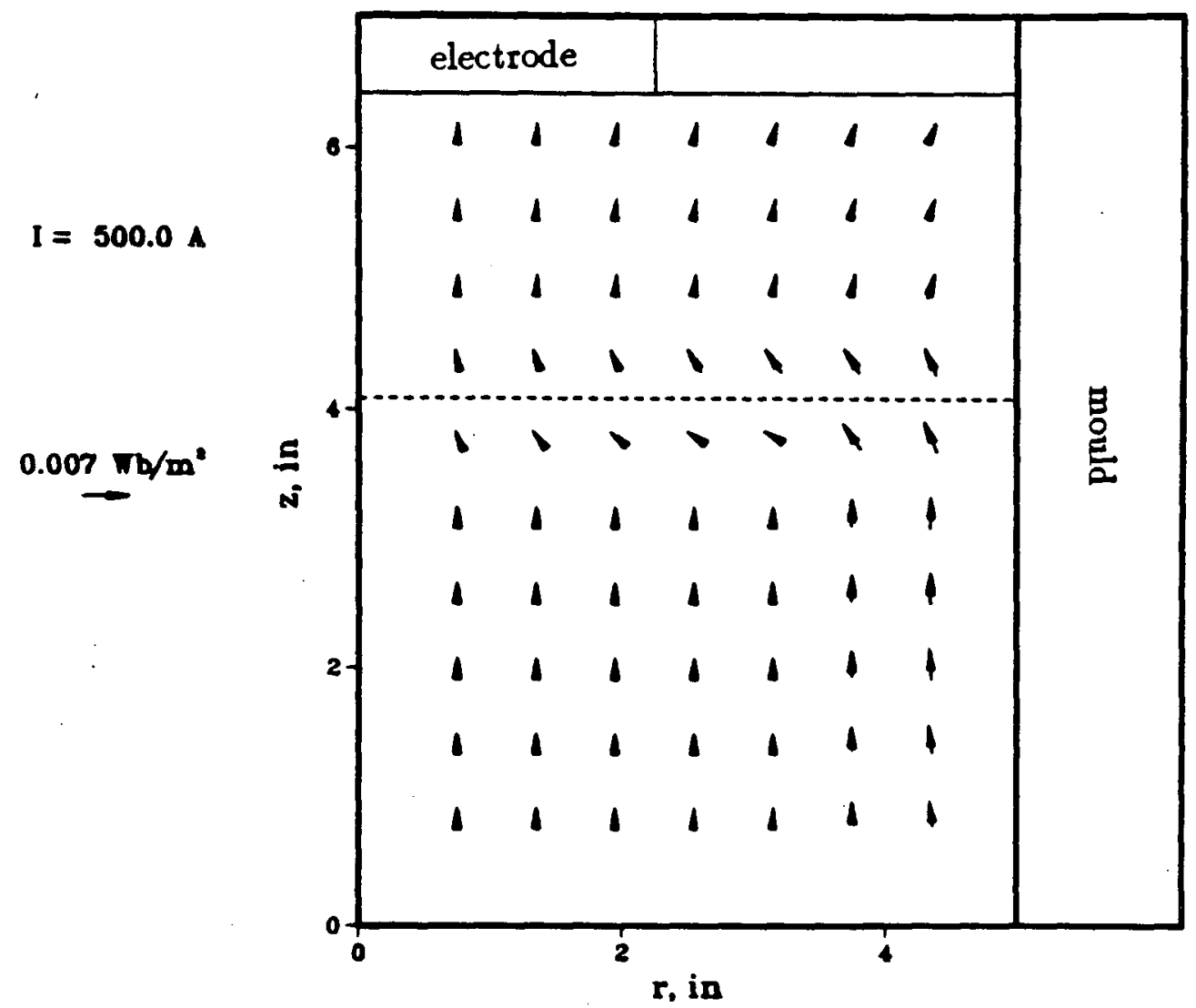

Figure 4.25: Magnetic field distribution obtained when the coil has only 2 turns. 


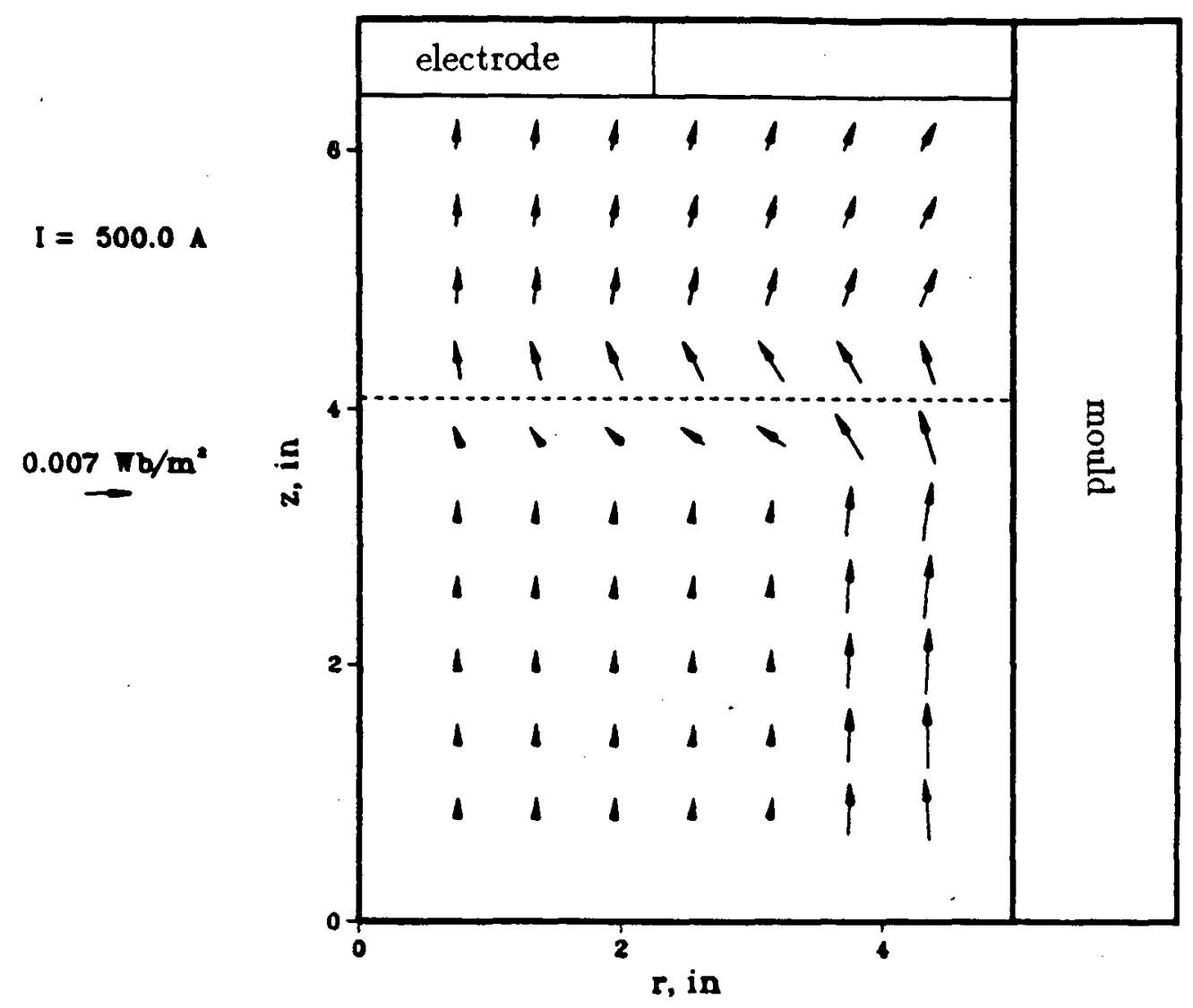

Figure 4.26: Magnetic field distribution obtained for the coil geometry shown. 


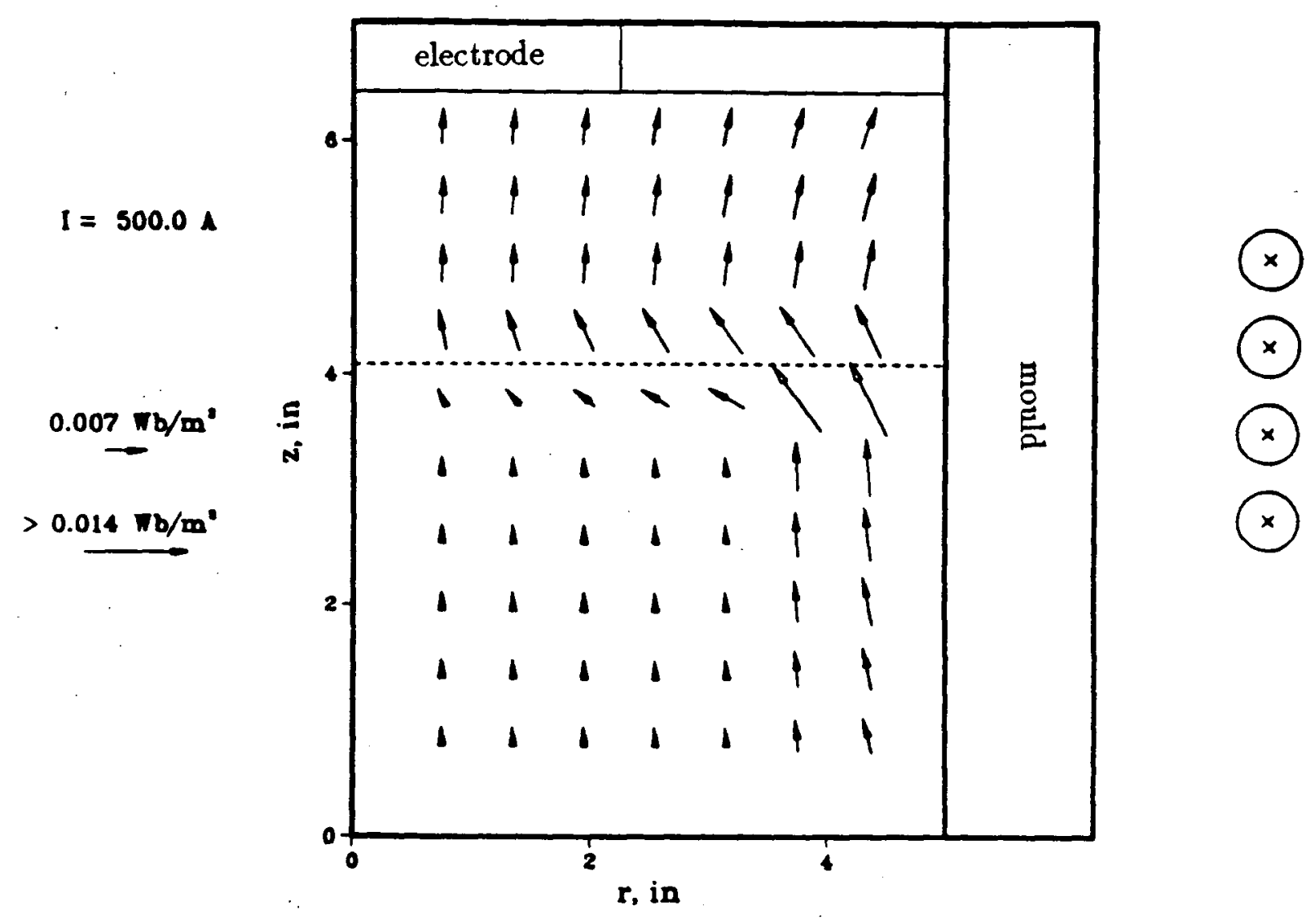

Figure 4.27: Magnetic field distribution obtained for the coil geometry shown. 


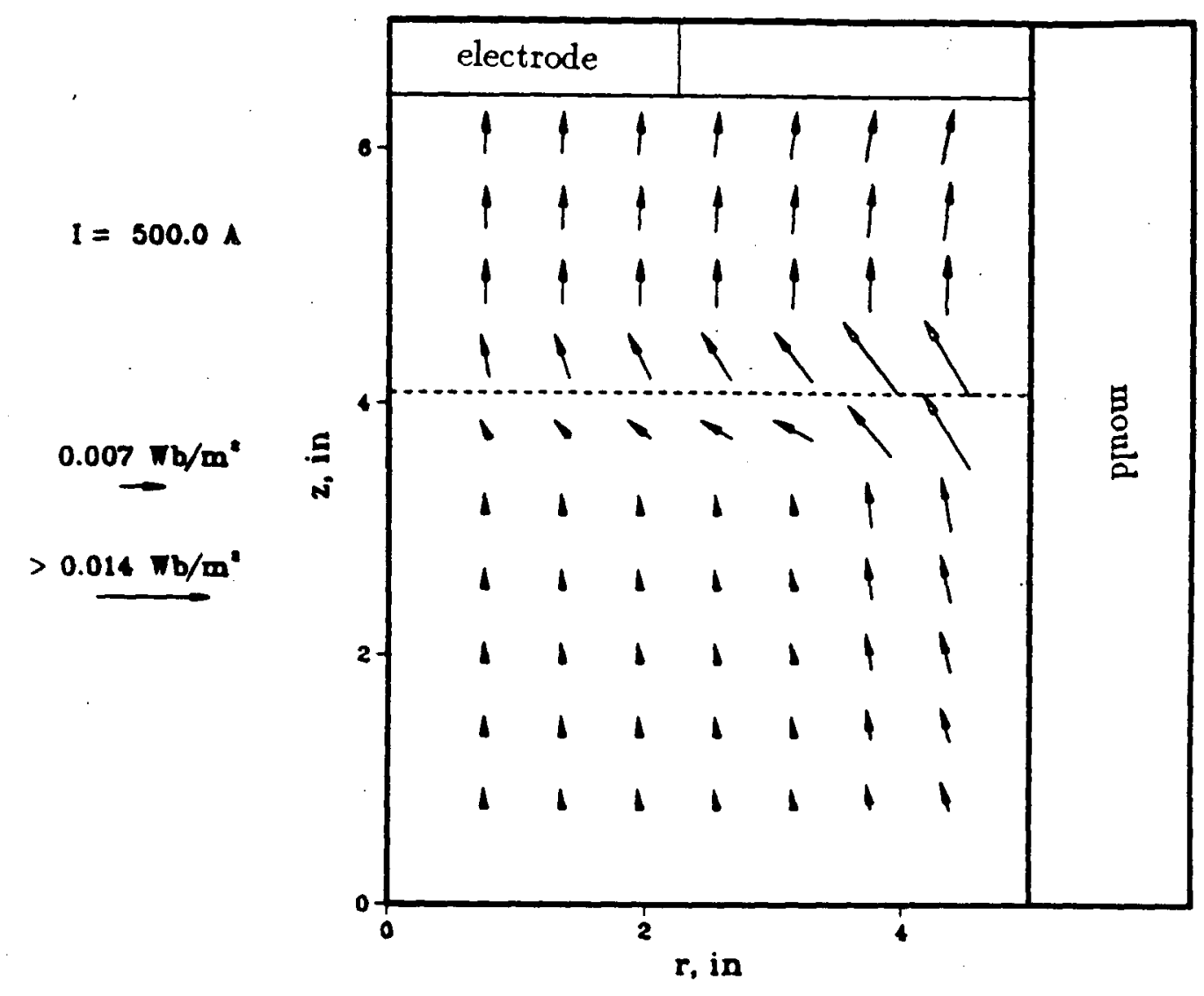

(x) (x)

Figure 4.28: Magnetic field distribution obtained for the coil geometry shown. 


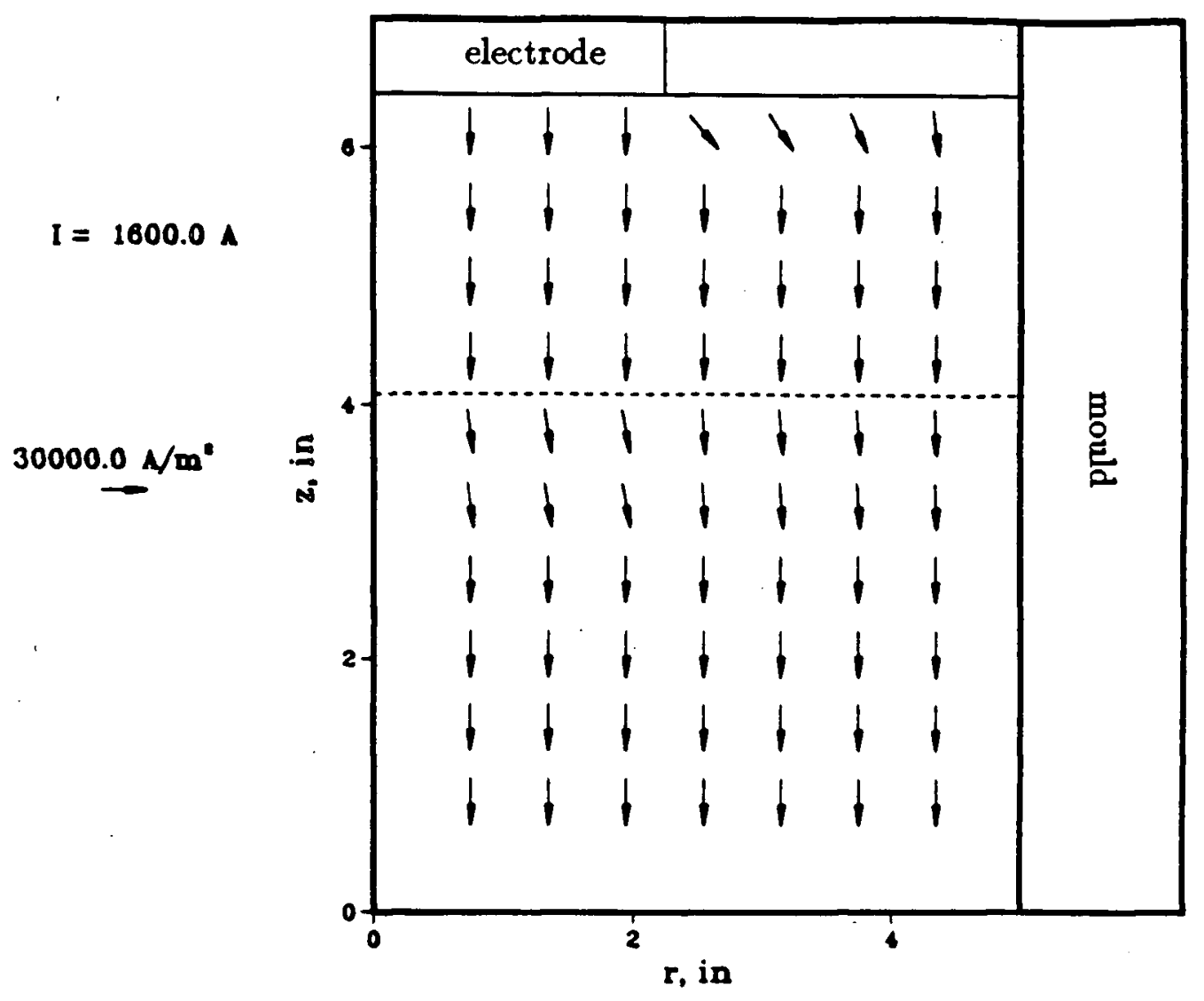

Figure 4.29: Current density distribution used in the electromagnetic body force calculations. 

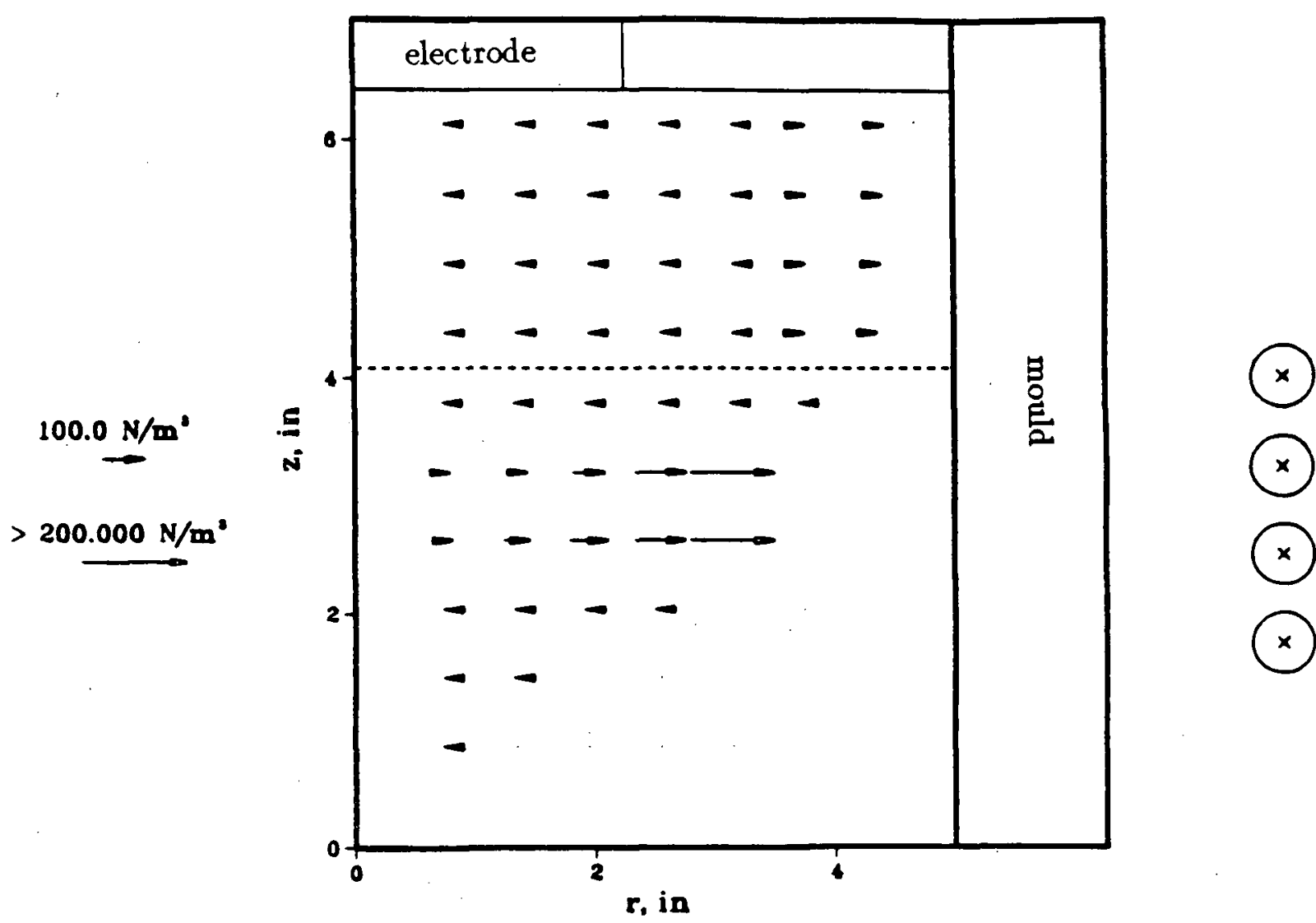

Figure 4.30: Electromagnetic body force distribution resulting from the interaction of the fields shown in figs. 4.29 and 4.19. All vectors are projections in the $r-z$ plane. 

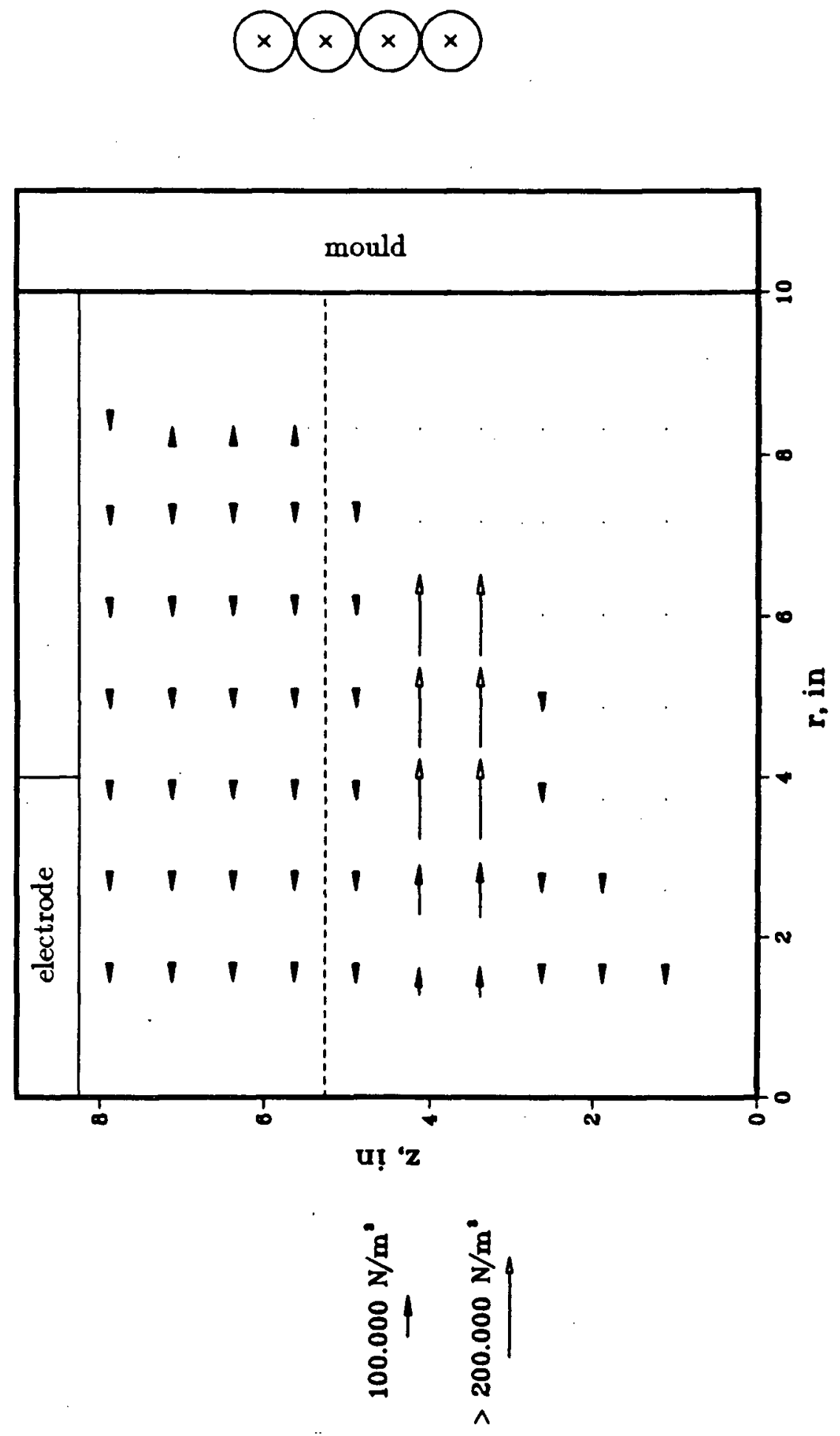

Figure 4.31: Electromagnetic body force distribution for a $500 \mathrm{~mm}$ dia. ingot. Melting current is $10 \mathrm{kA}$. All vectors are projections in the $r-z$ plane. 

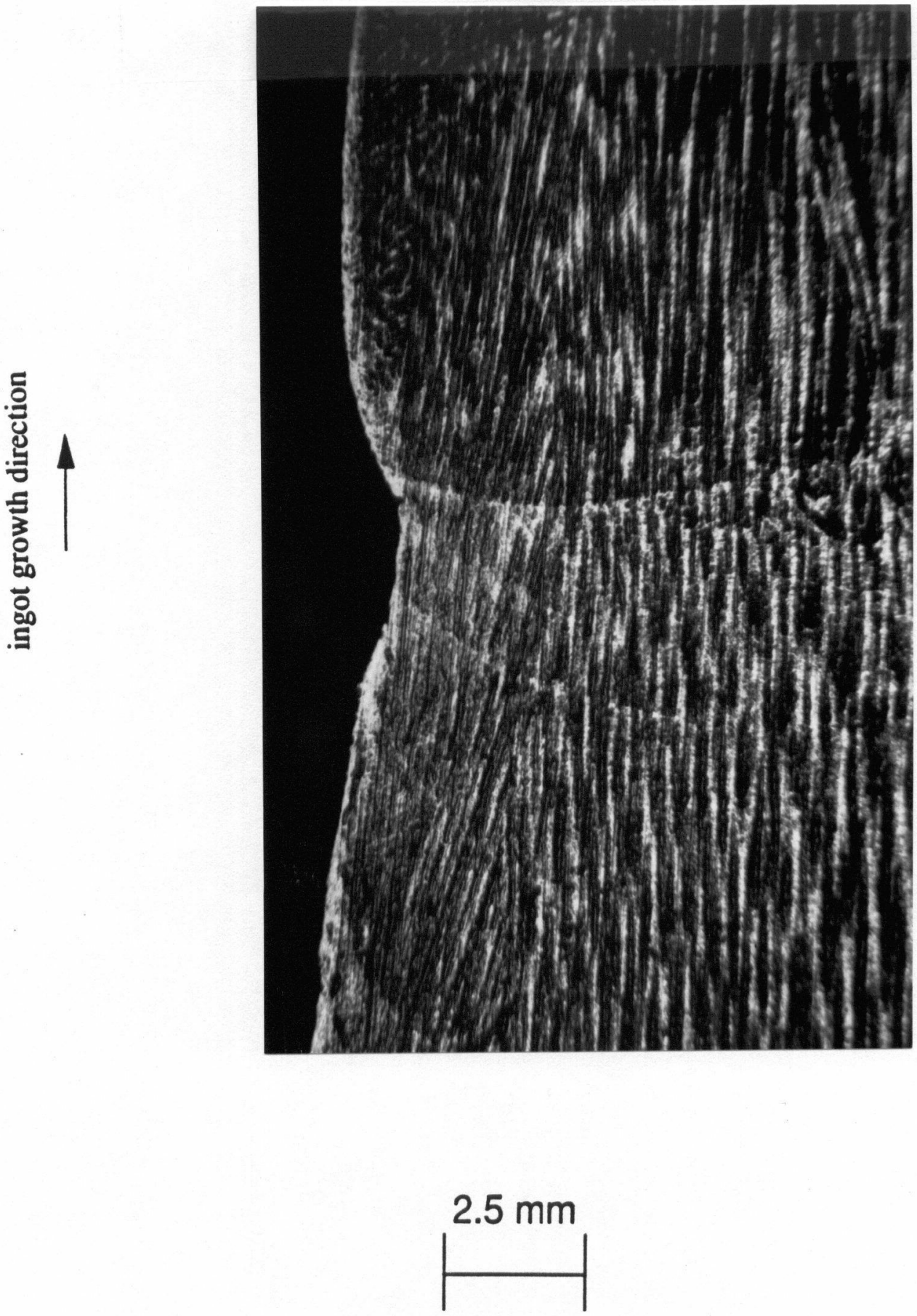

Figure 4.32: Detail of the ingot surface showing the effect of slag rotation. 


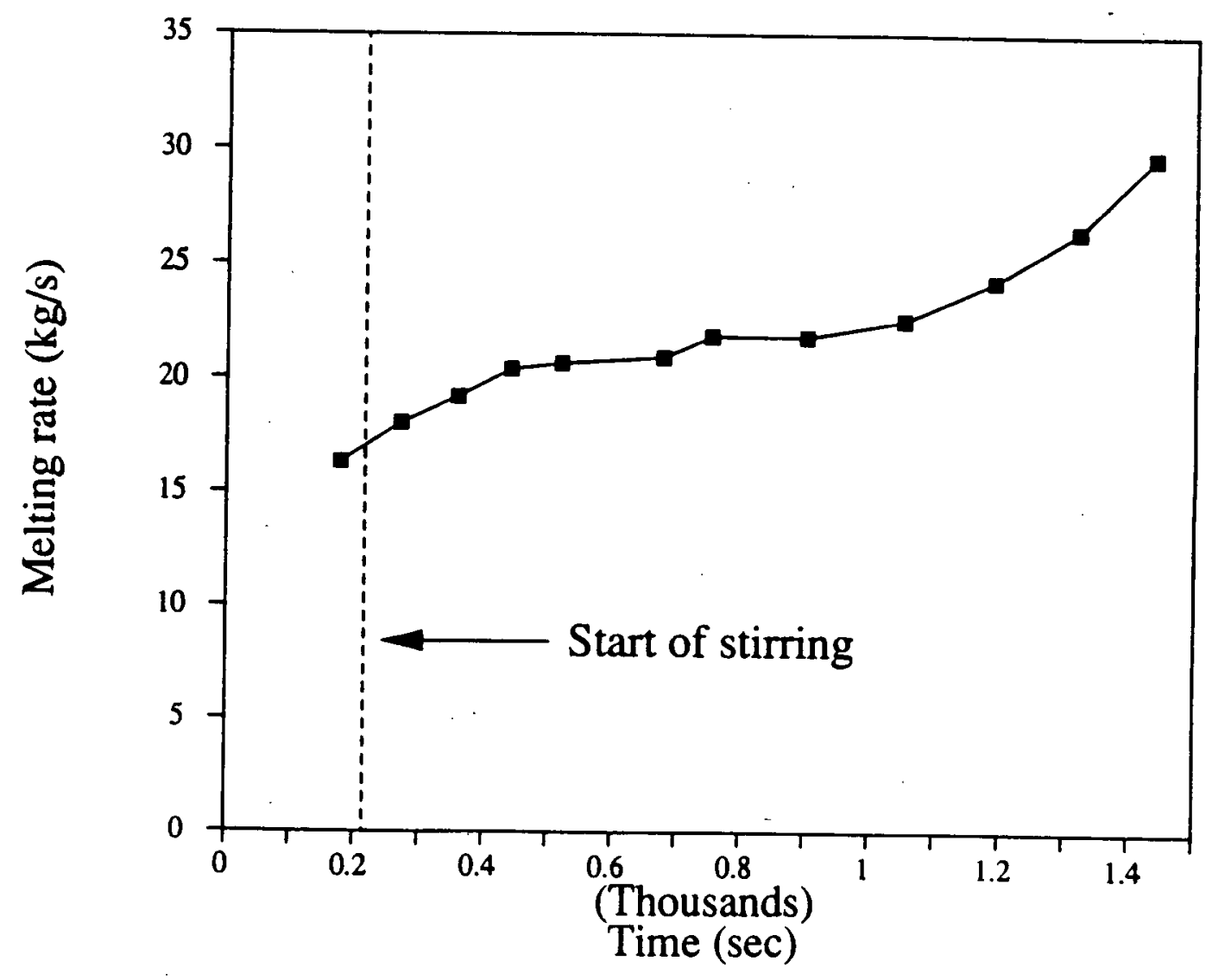

Figure 4.33: Melting rate as a function of time for ingot XI (stirred). 


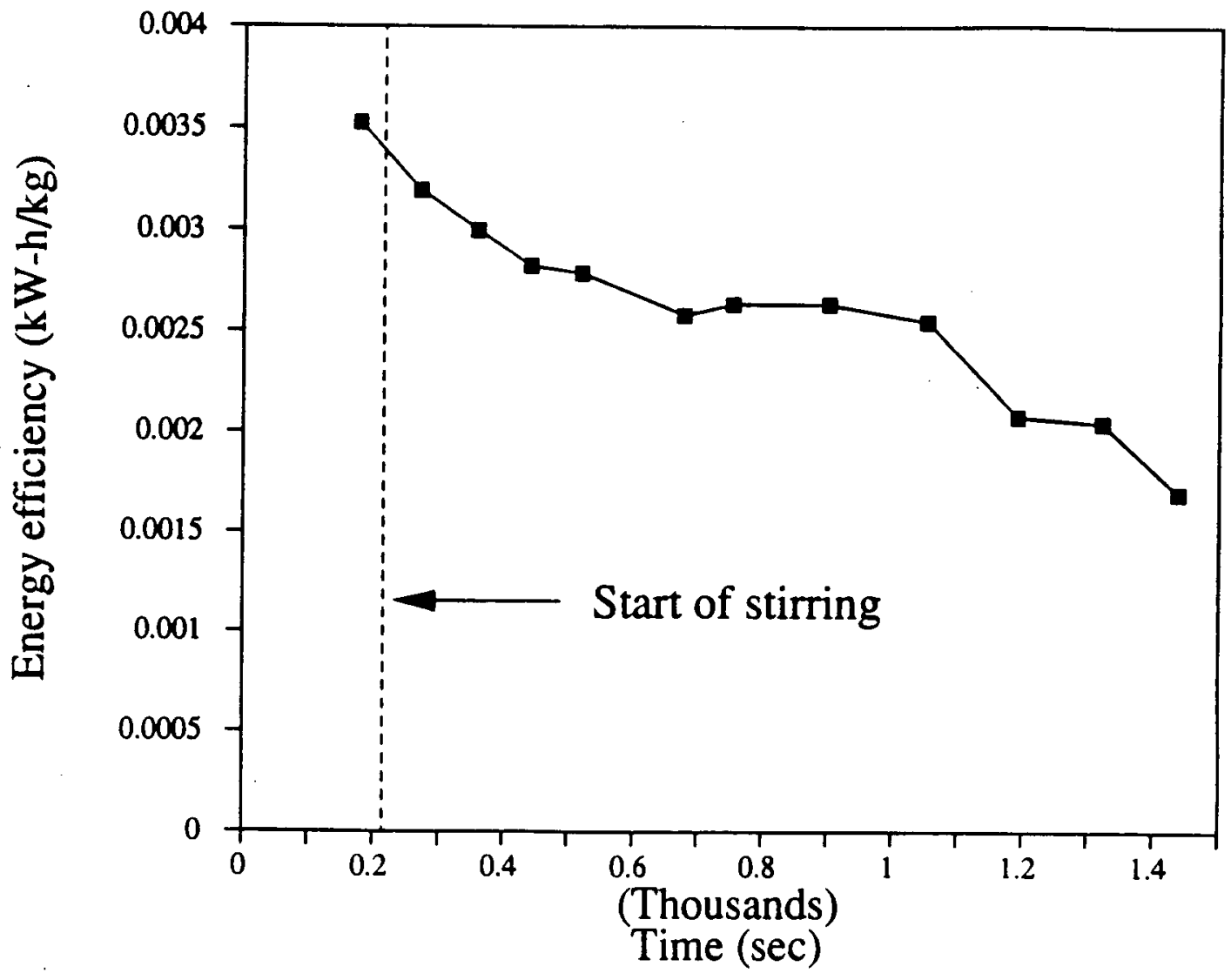

Figure 4.34: Energy efficiency as a function of time for ingot XI (stirred). 


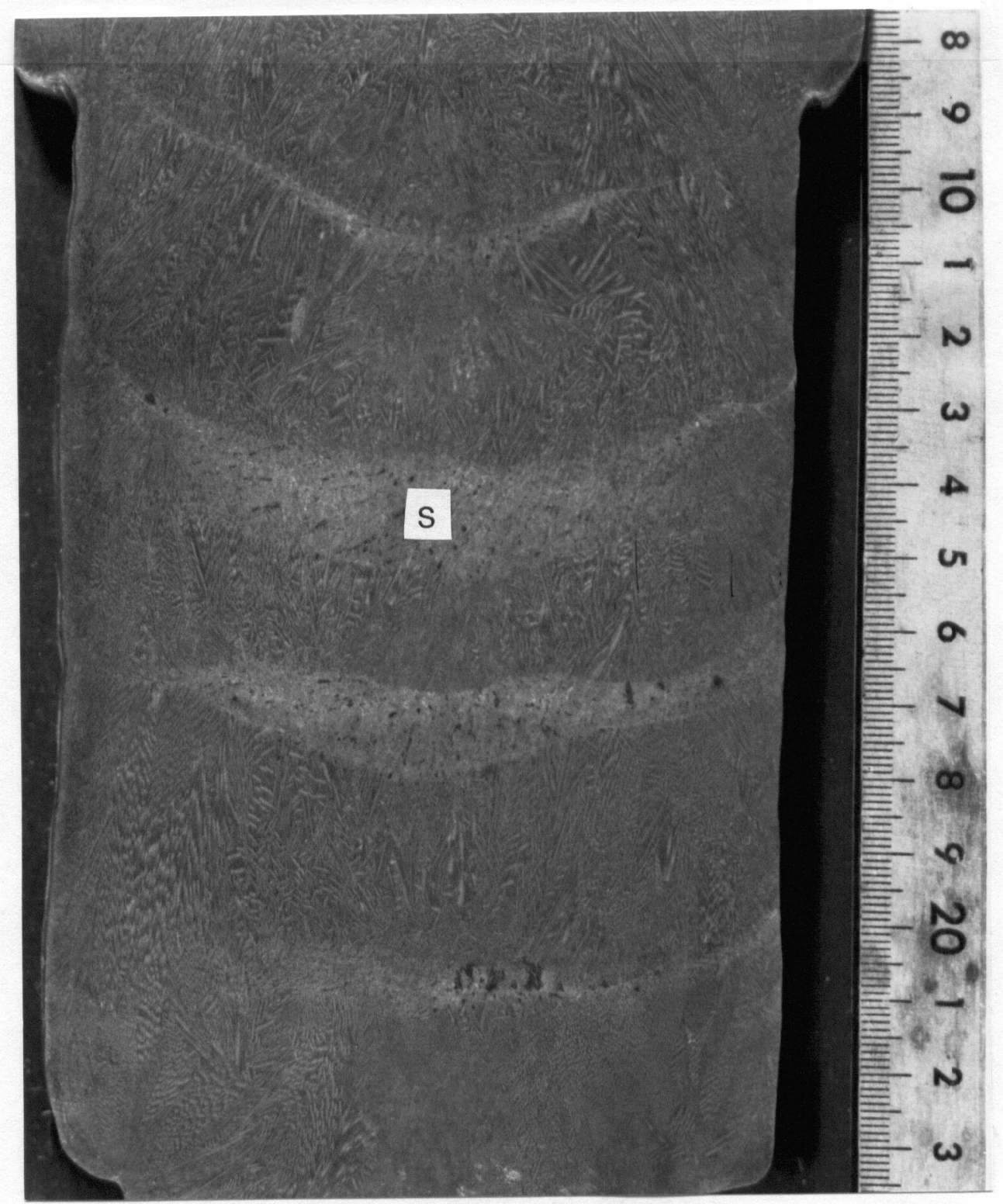

Figure 4.35: Photomacrograph of ingot II. Unsteady-state stirring, I $=500 \mathrm{~A}$. A typical stirred zone is marked with an 'S'. 


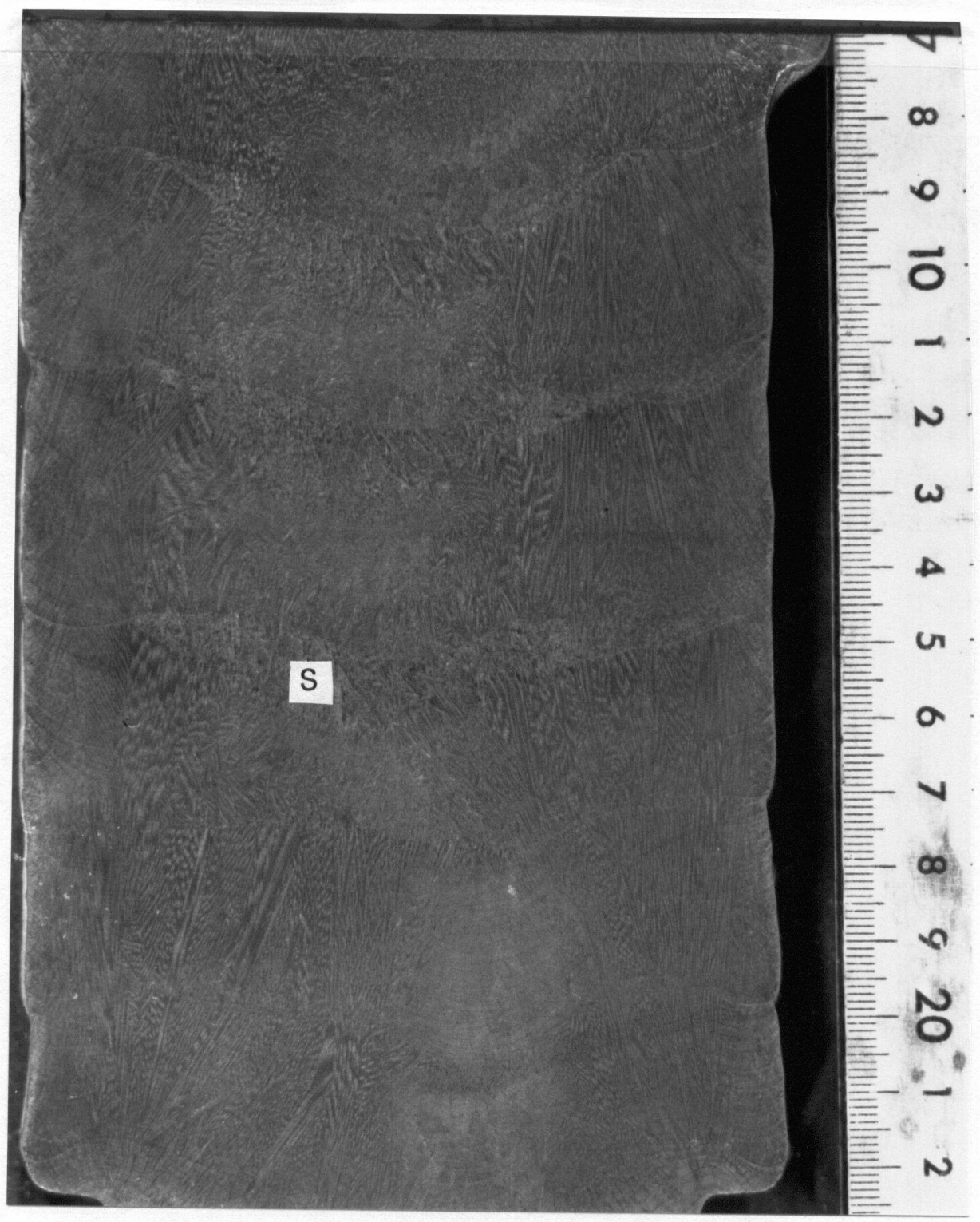

Figure 4.36: Photomacrograph of ingot III. Unsteady-state stirring, I = $350 \mathrm{~A}$. A typical stirred zone is marked with an 'S'. 


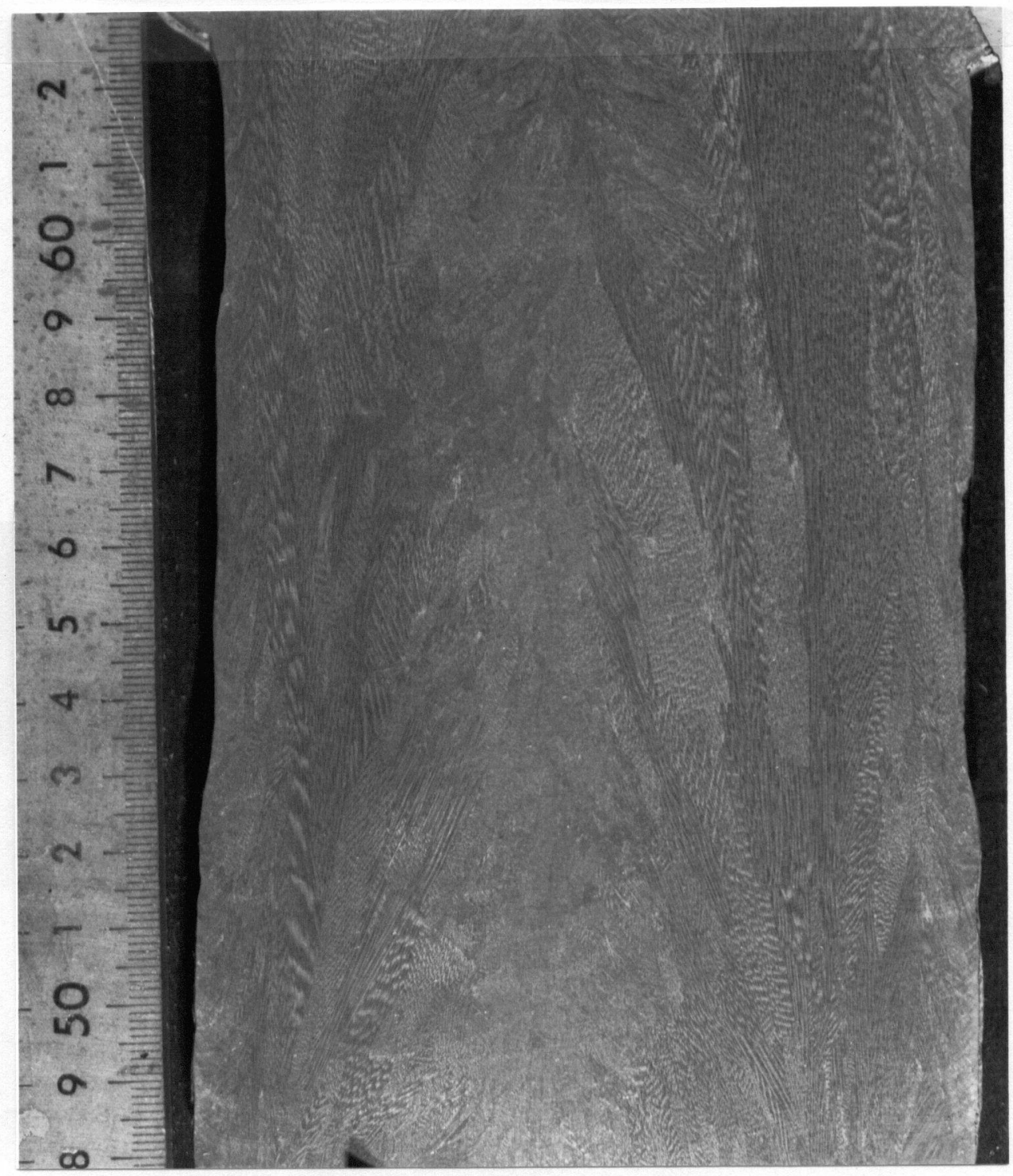

Figure 4.37: Photomacrograph of ingot IV. Unsteady-state stirring, $\mathrm{I}=100 \mathrm{~A}$. 


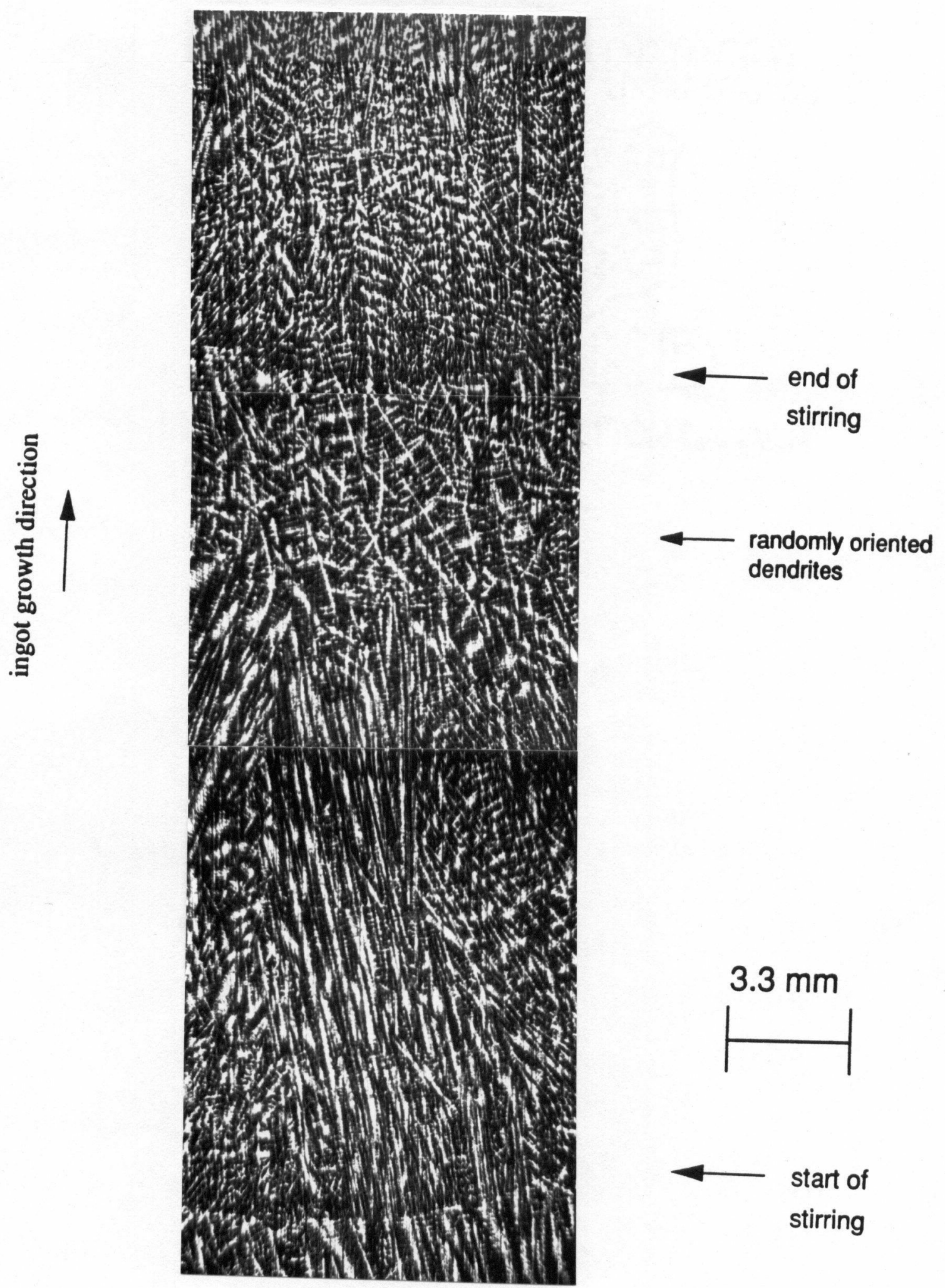

Figure 4.38: Detail of the stirred zone in ingot III. 


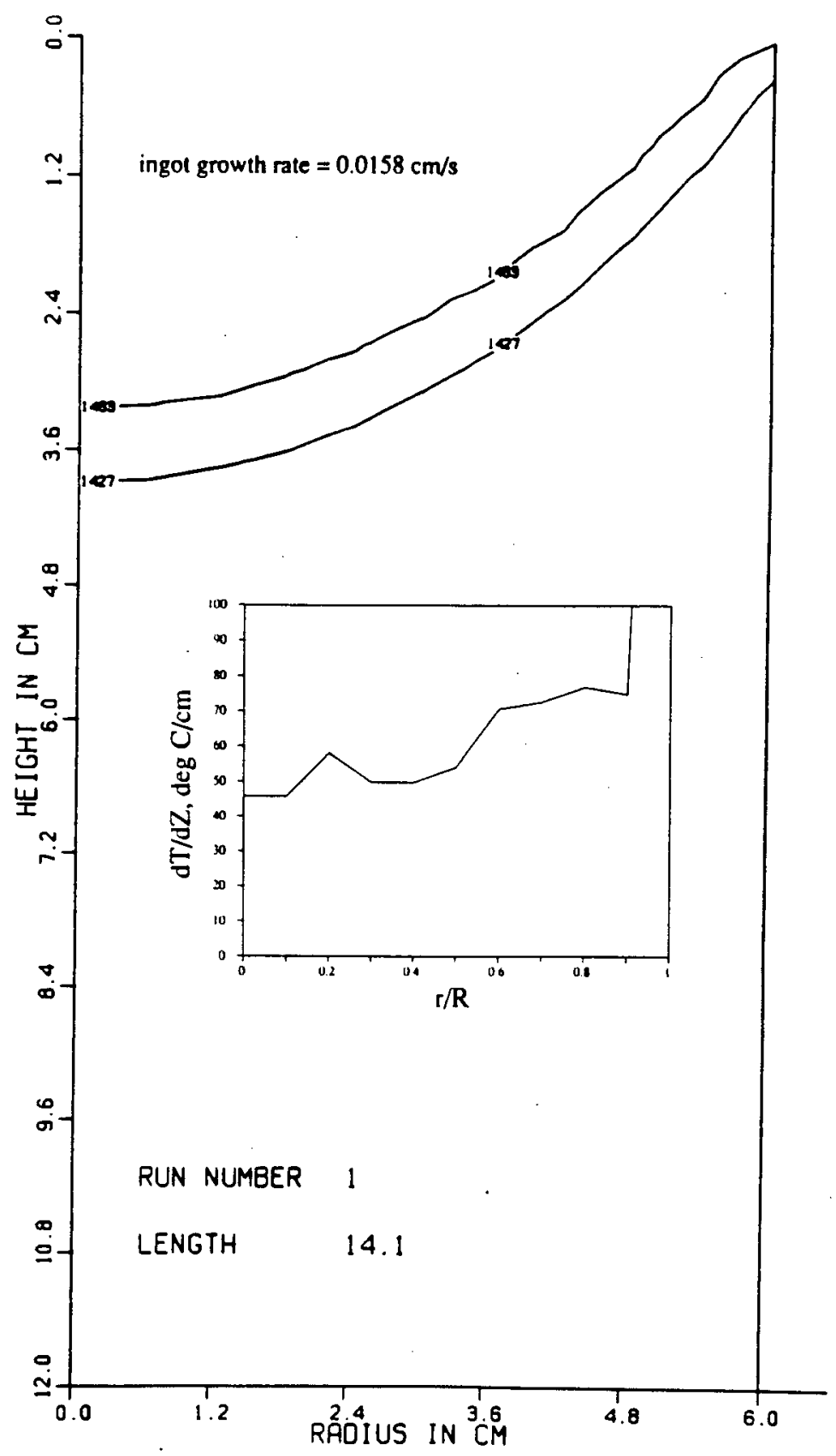

Figure 4.39: Mushy zone height, temperature gradient and solidification rate for the experimental conditions. Obtained with a two-dimensional, steady-state heat transfer model [131]. 


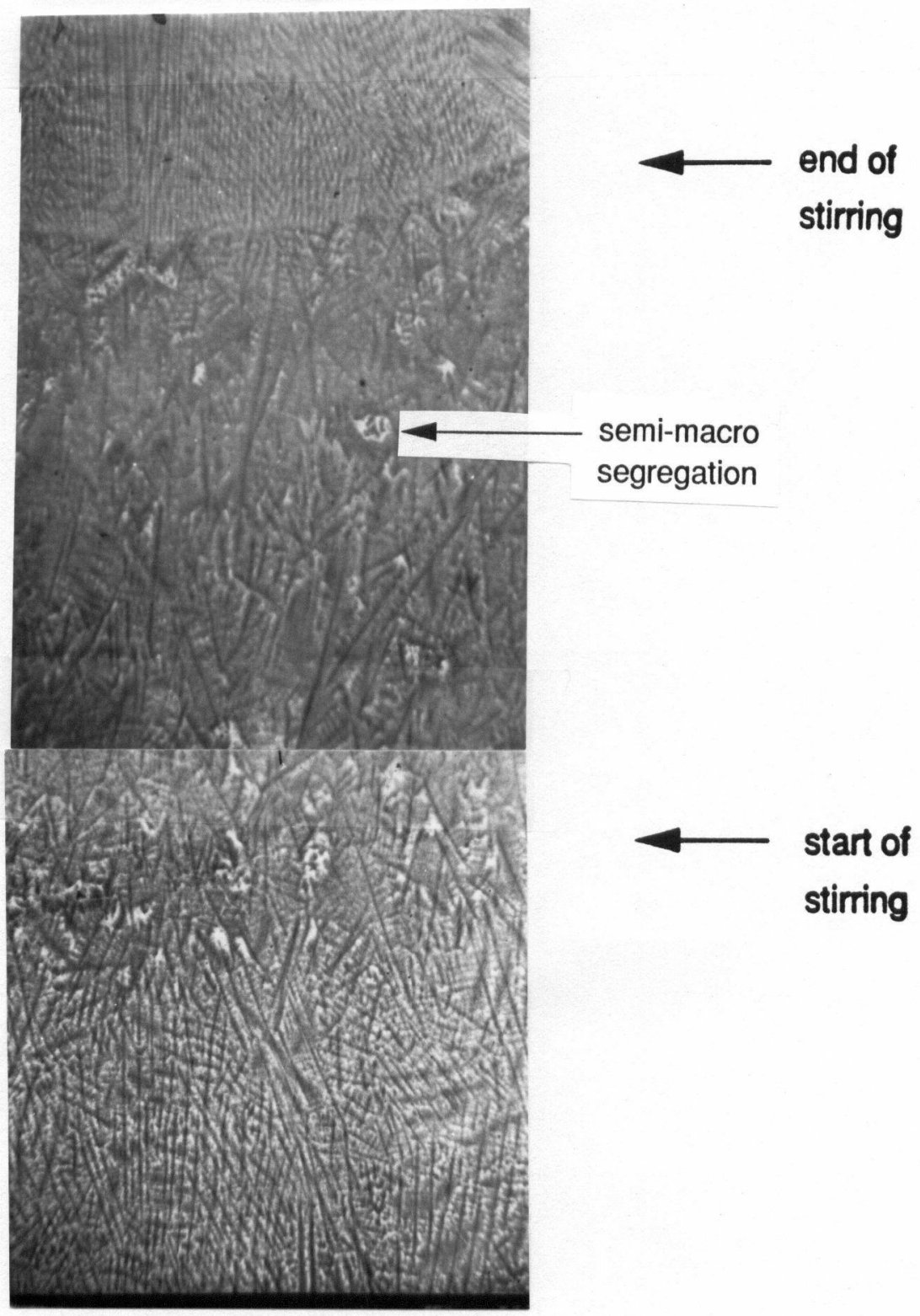

\section{$3.5 \mathrm{~mm}$}

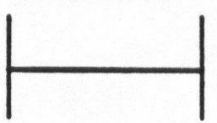

Figure 4.40: Microphotograph of the stirred zone in ingot II. 


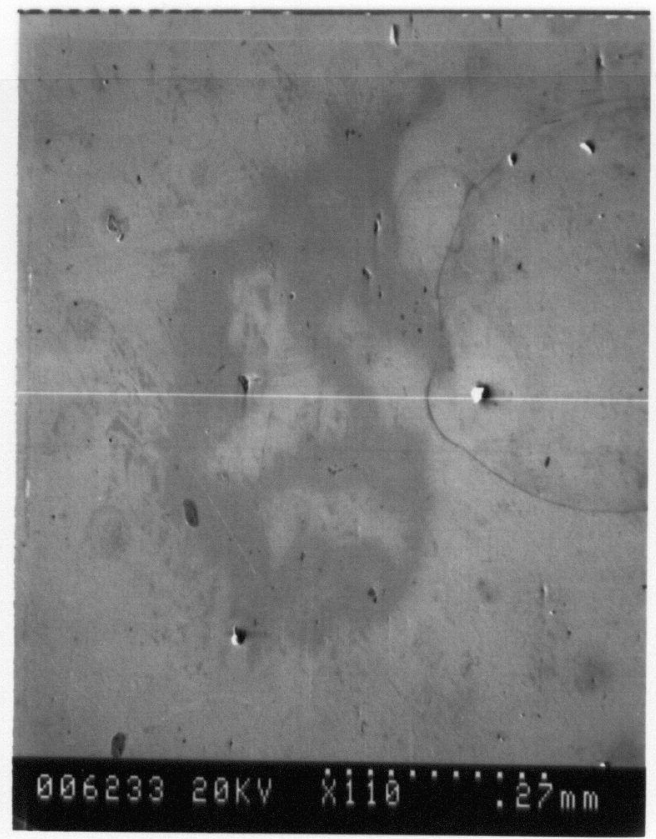

(a)

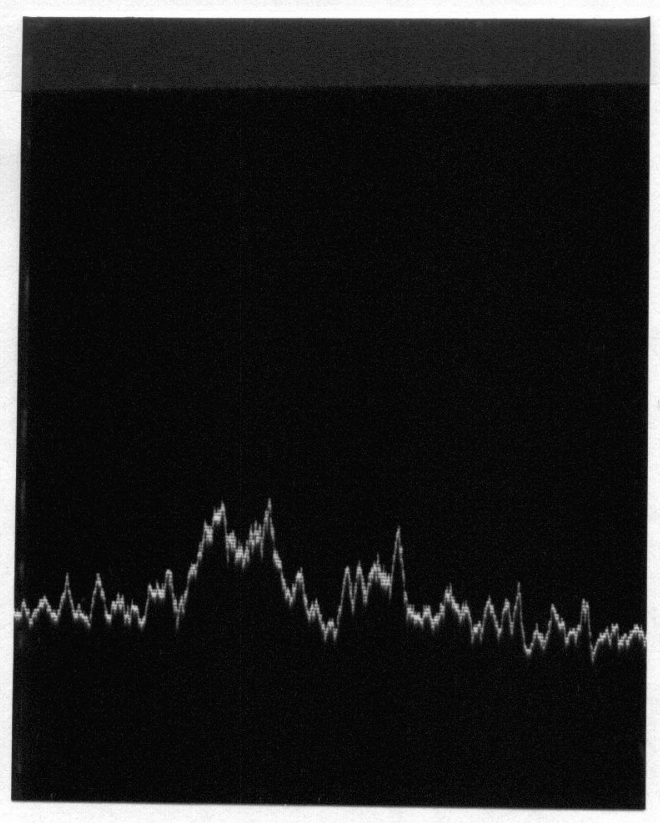

(b)

Figure 4.41: Analysis of a segregated area in the stirred zone in ingot II. (a) SEM picture. (b) Phosphorus segregation. 


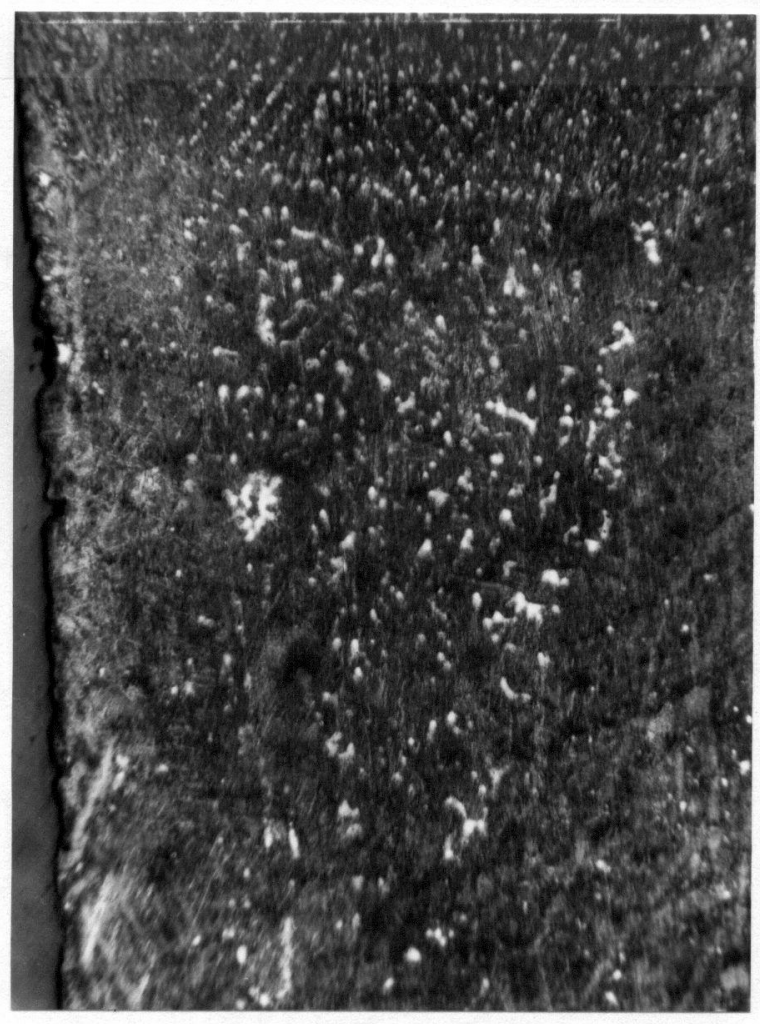

\section{end of} stirring

\section{$2.8 \mathrm{~mm}$}

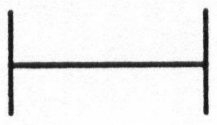

Figure 4.42: Photomicrograph of the stirred zone in ingotII showing an area perpendicular to the area shown in fig. 4.40 . 


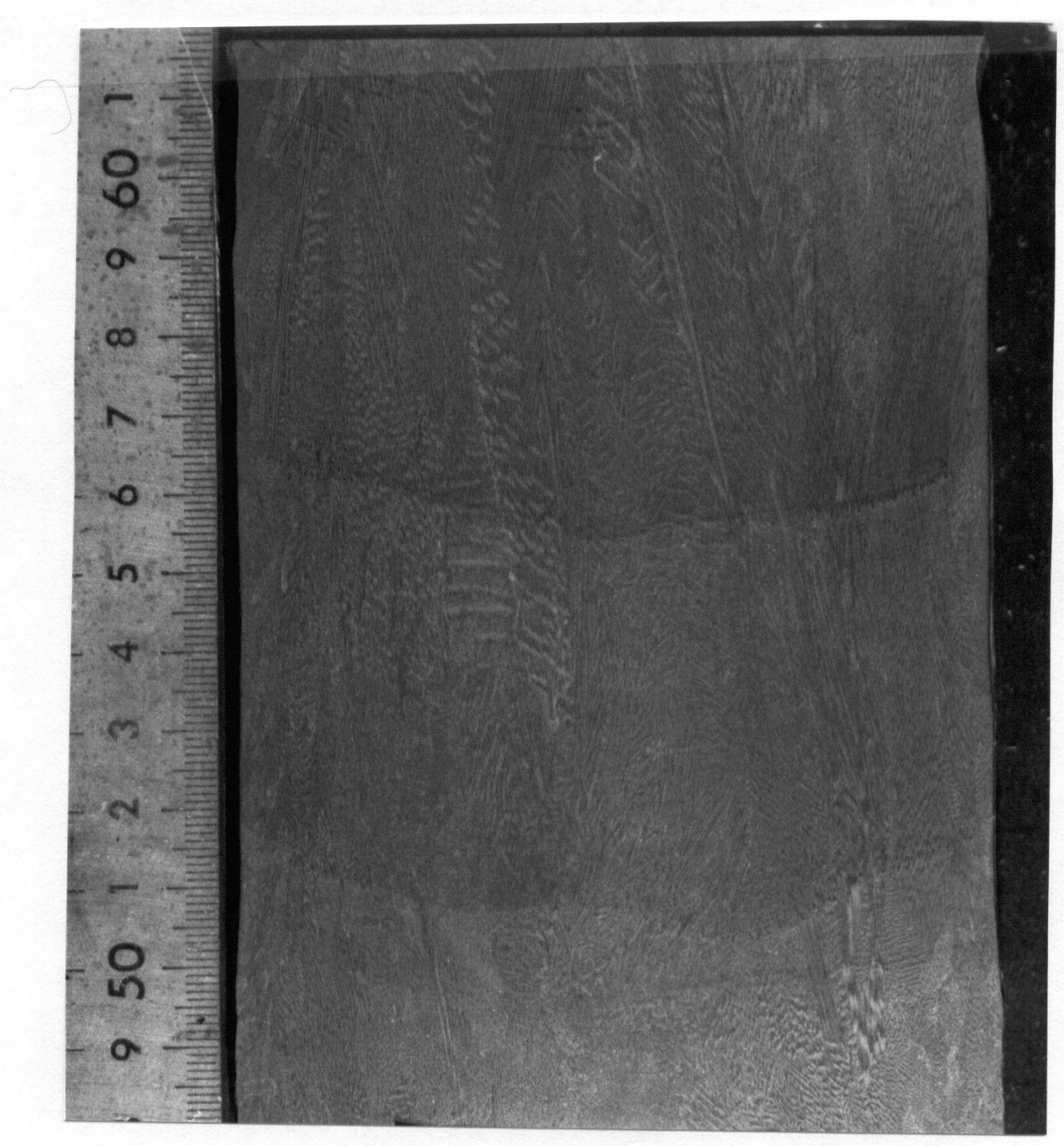

Figure 4.43: Photomacrograph of ingot V. Steady-state stirring, I $=350 \mathrm{~A}$. 


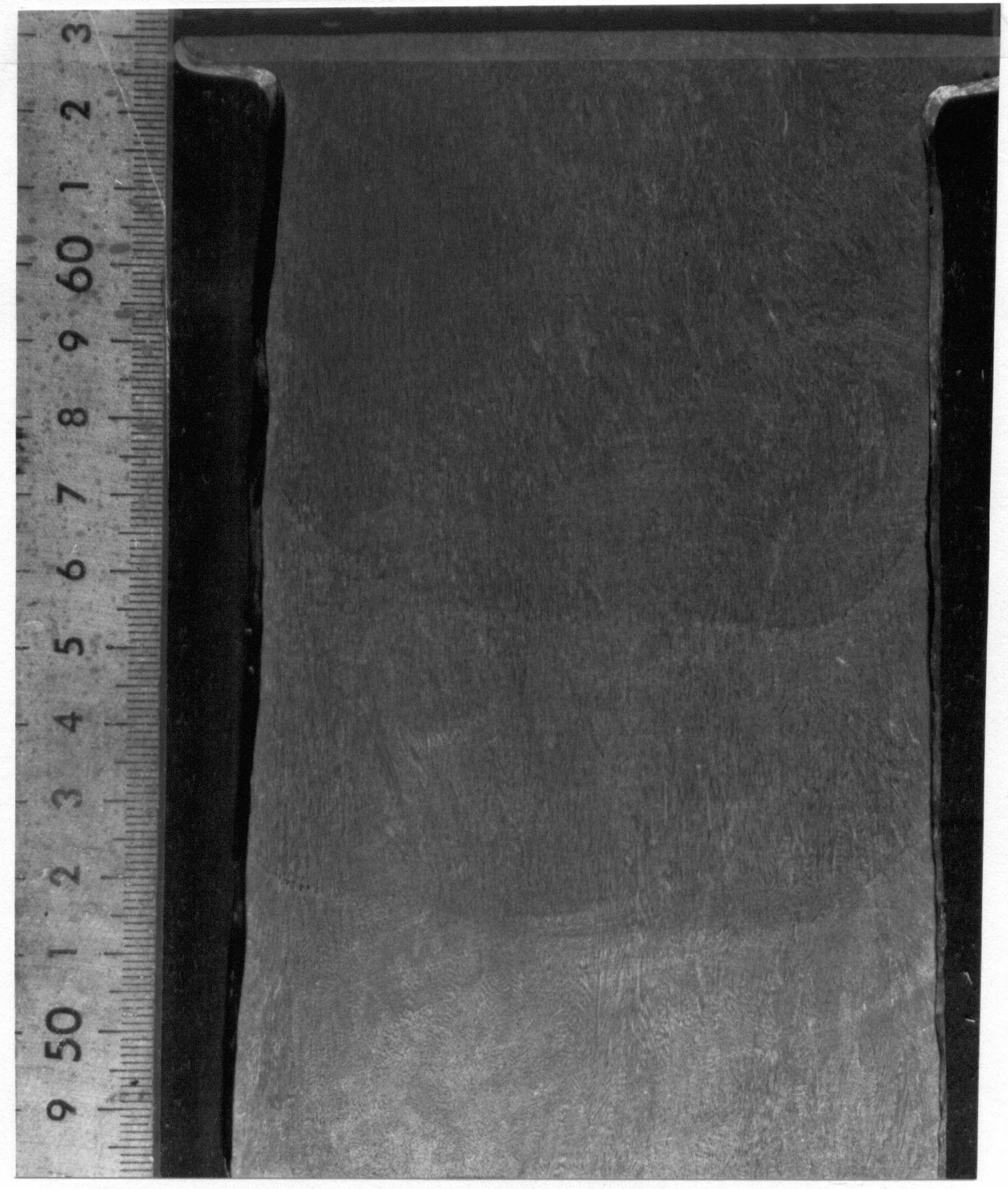

Figure 4.44: Photomacrograph of ingot VI. Steady-state stirring, I $=350 \mathrm{~A}$. 


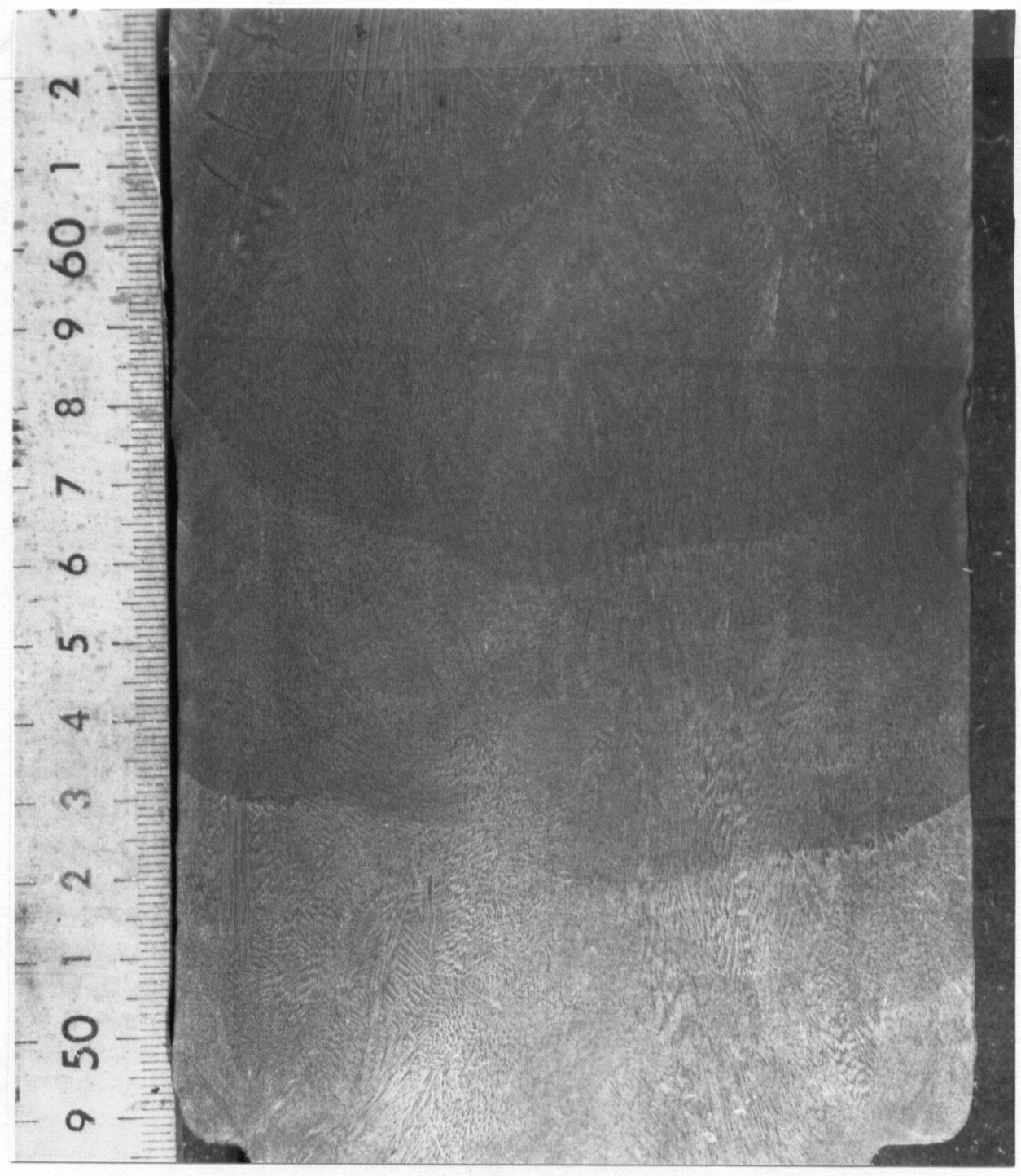

Figure 4.45: Photomacrograph of ingot VII. Unsteady- and steady-state stirring, $\mathrm{I}=250 \mathrm{~A}$. 


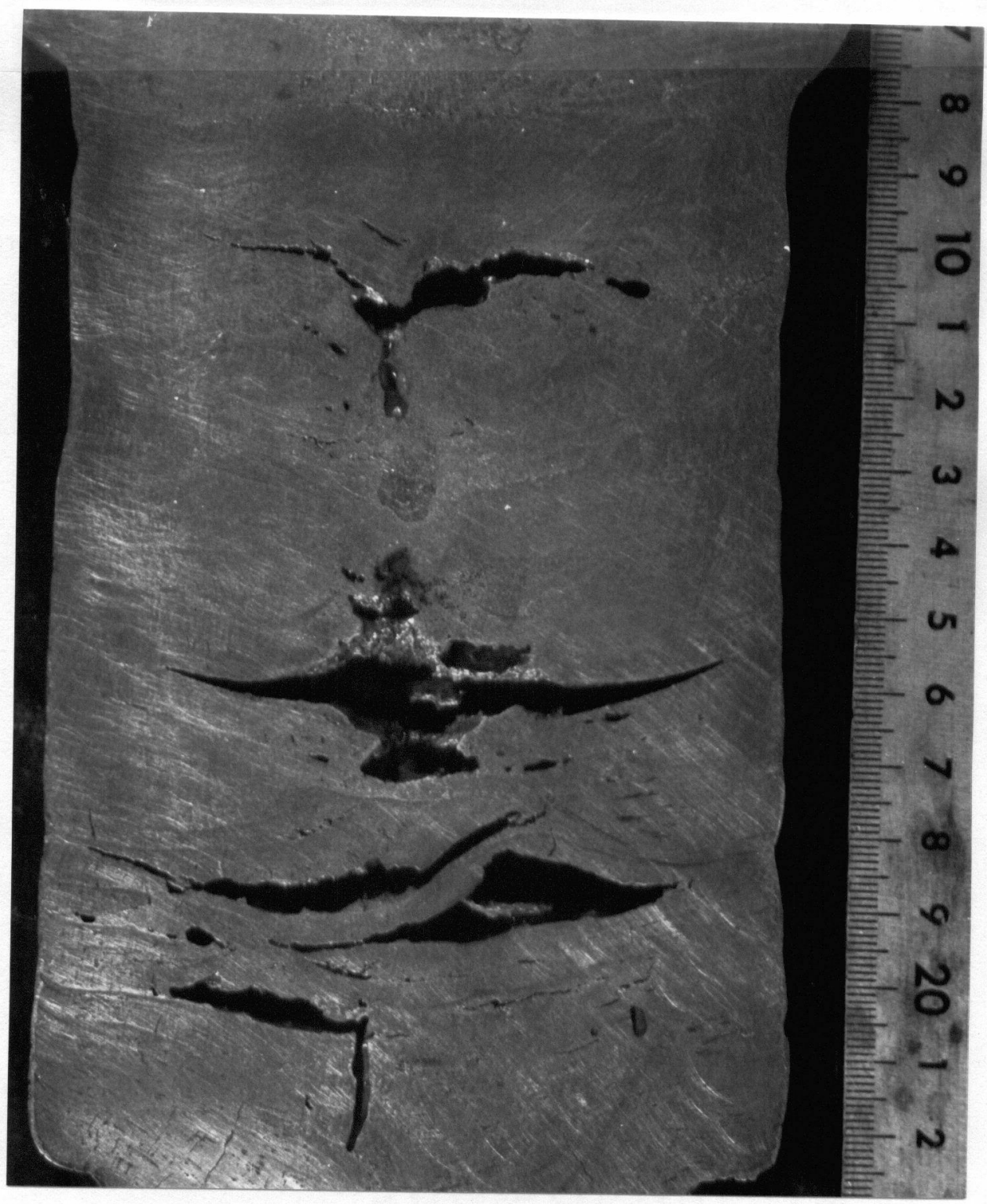

Figure 4.46: Photomacrograph of ingot VIII. Cu-50 Fe, I = $350 \mathrm{~A}$. 


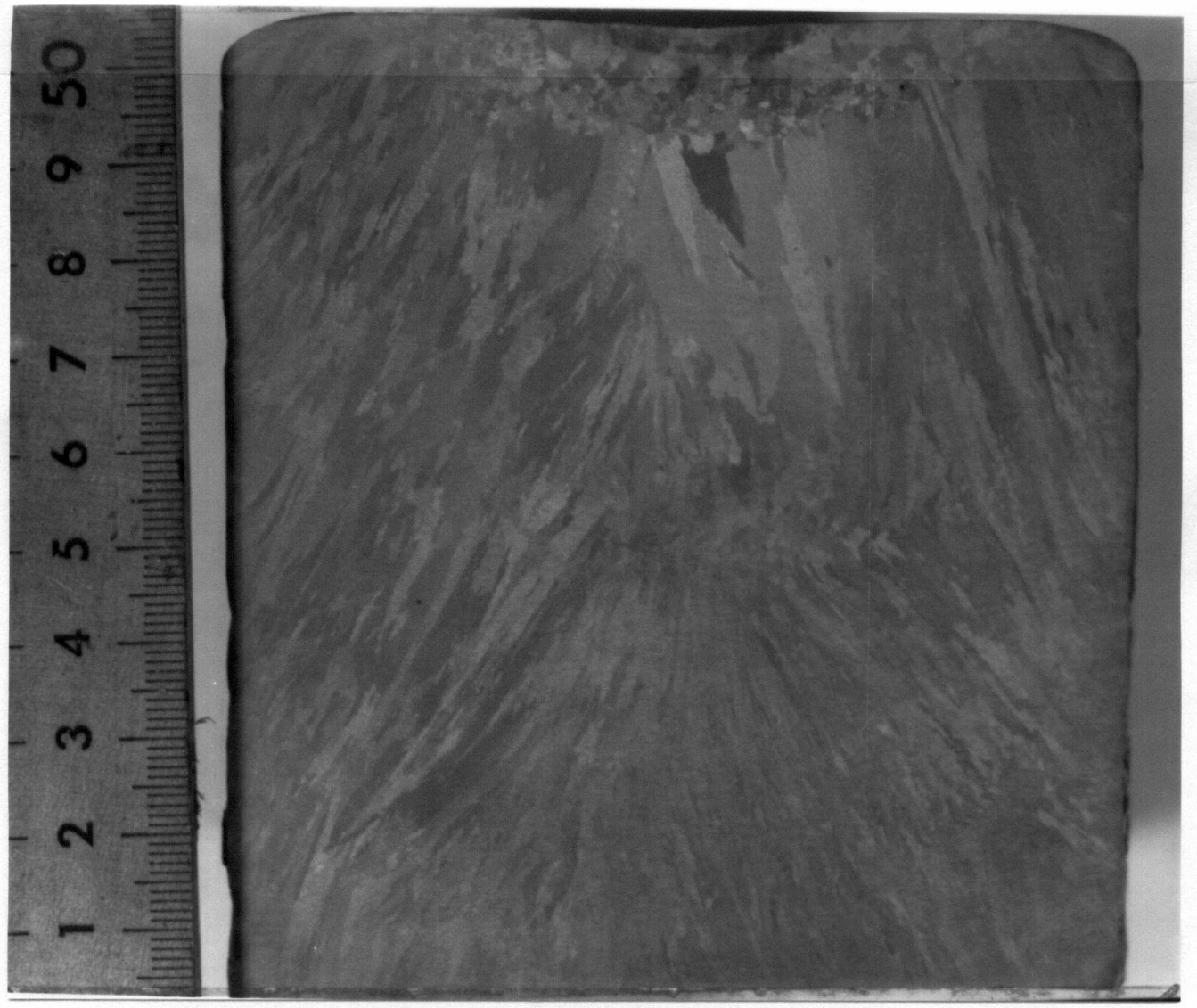

Figure 4.47: Photomacrograph of ingot IX. Inconel 718, I = 380 A. 


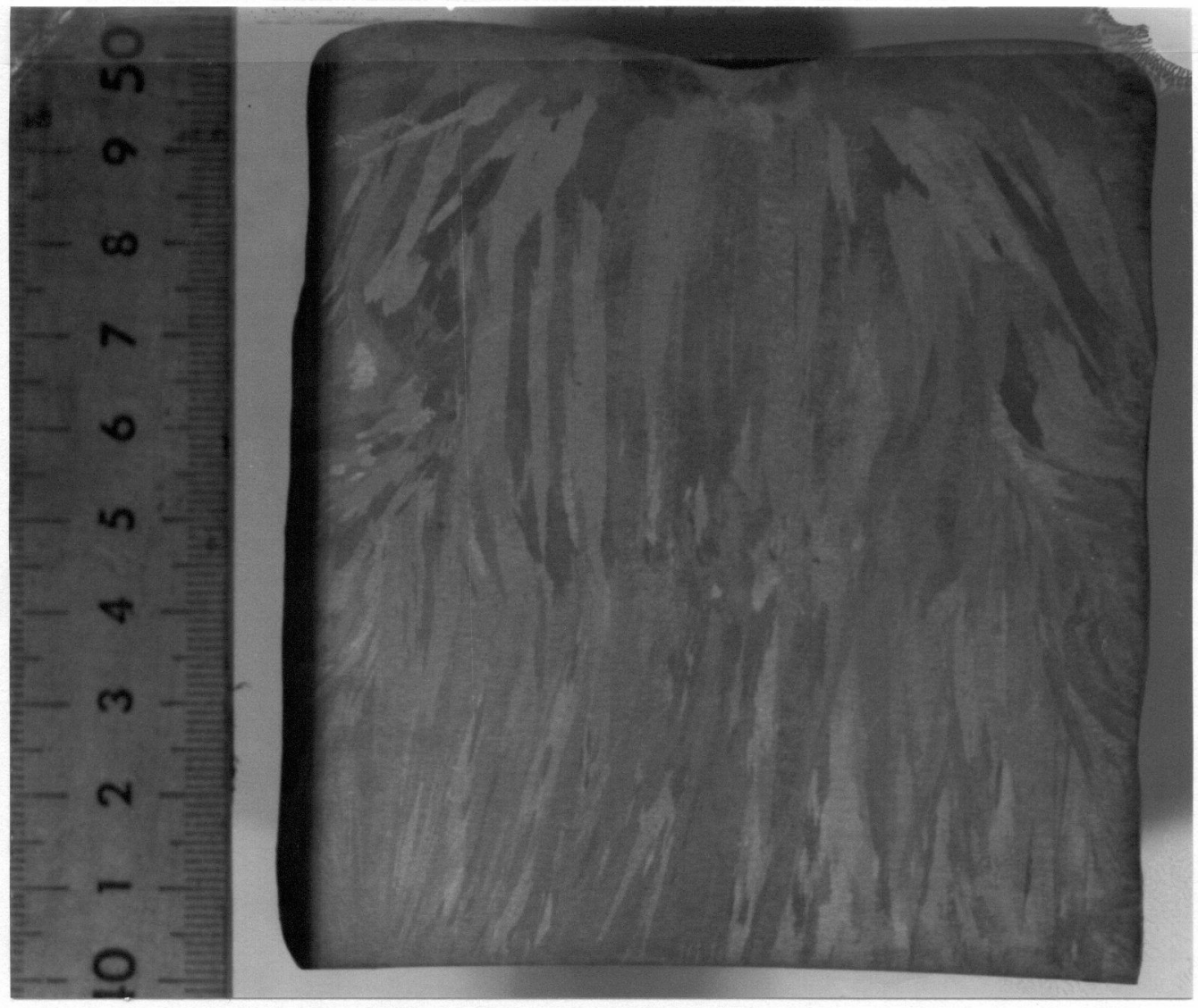

Figure 4.48: Photomacrograph of ingot X. Inconel 718, I = $380 \mathrm{~A}$, effect of slag composition. 

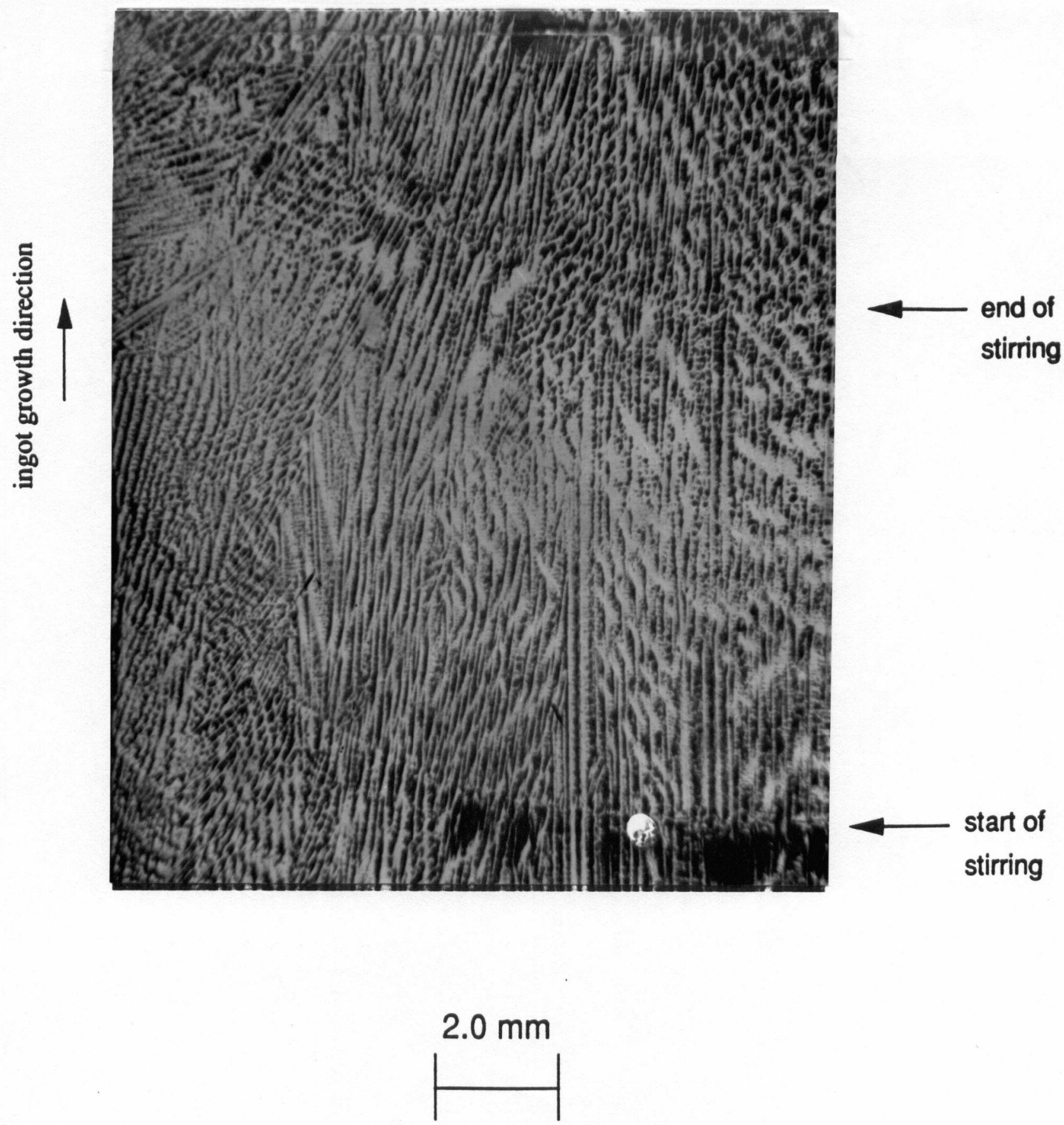

Figure 4.49: Microphotograph of sample 1 in fig. 3.18 . 


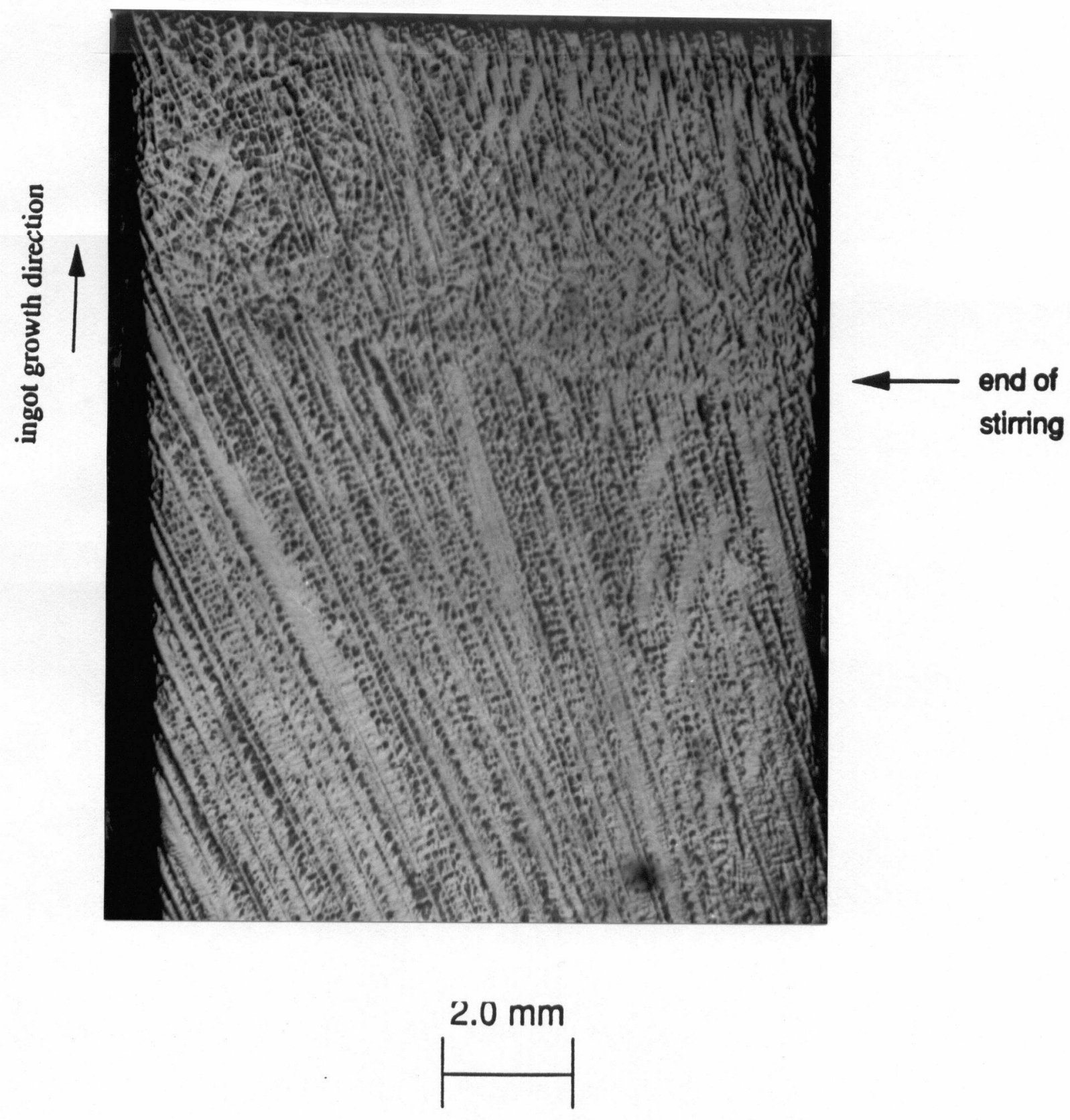

Figure 4.50: Microphotograph of sample 2 in fig. 3.18. 


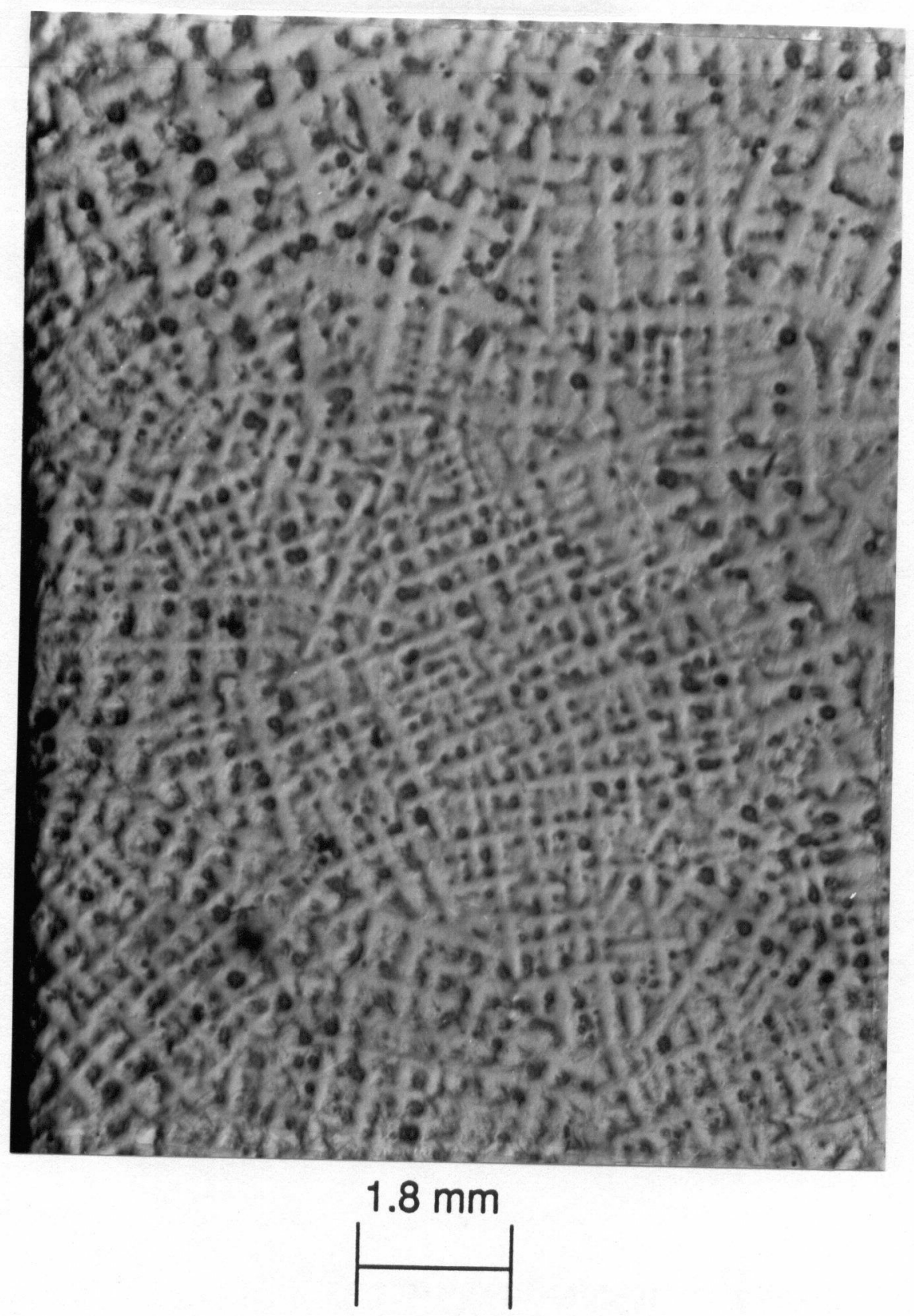

Figure 4.51: Microphotograph of sample 3 in fig. 3.18. 

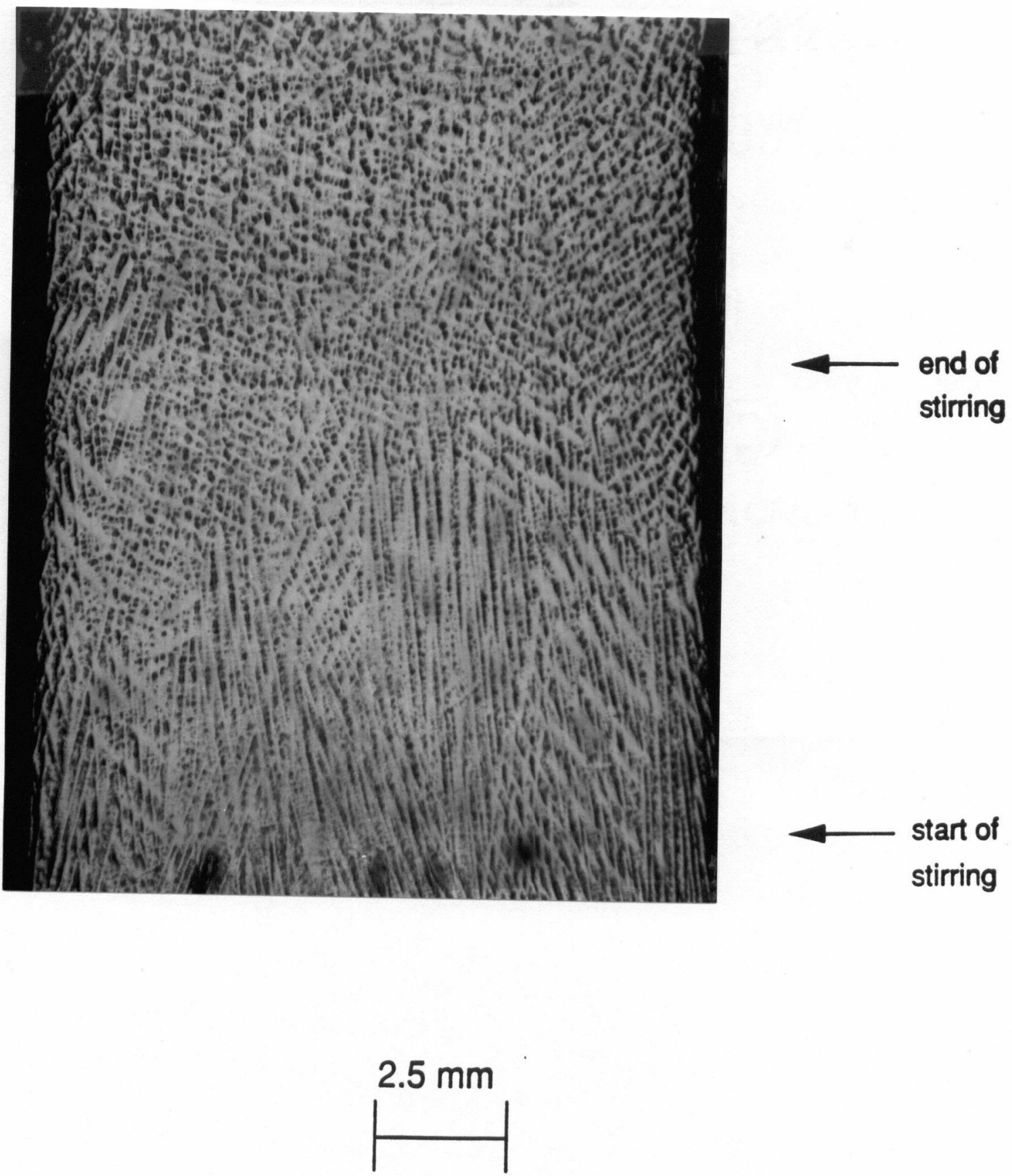

Figure 4.52: Microphotograph of sample 4 in fig. 3.18 . 


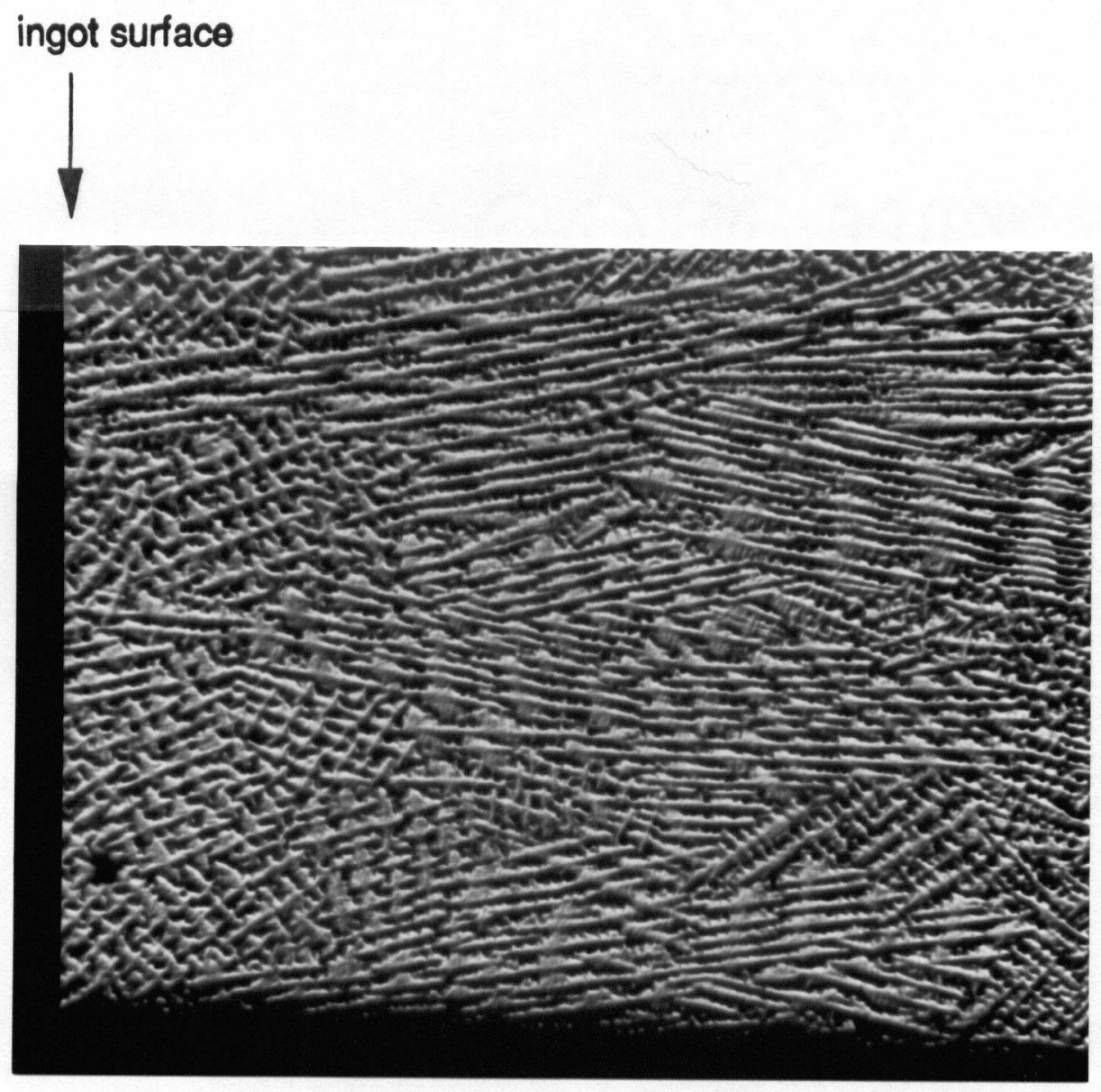

ingot radial direction

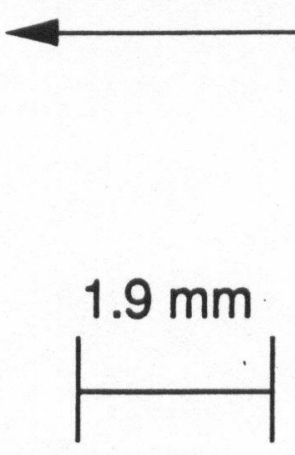

Figure 4.53: Microphotograph of a cross-sectional sample at the ingot surface (non stirred zone). 


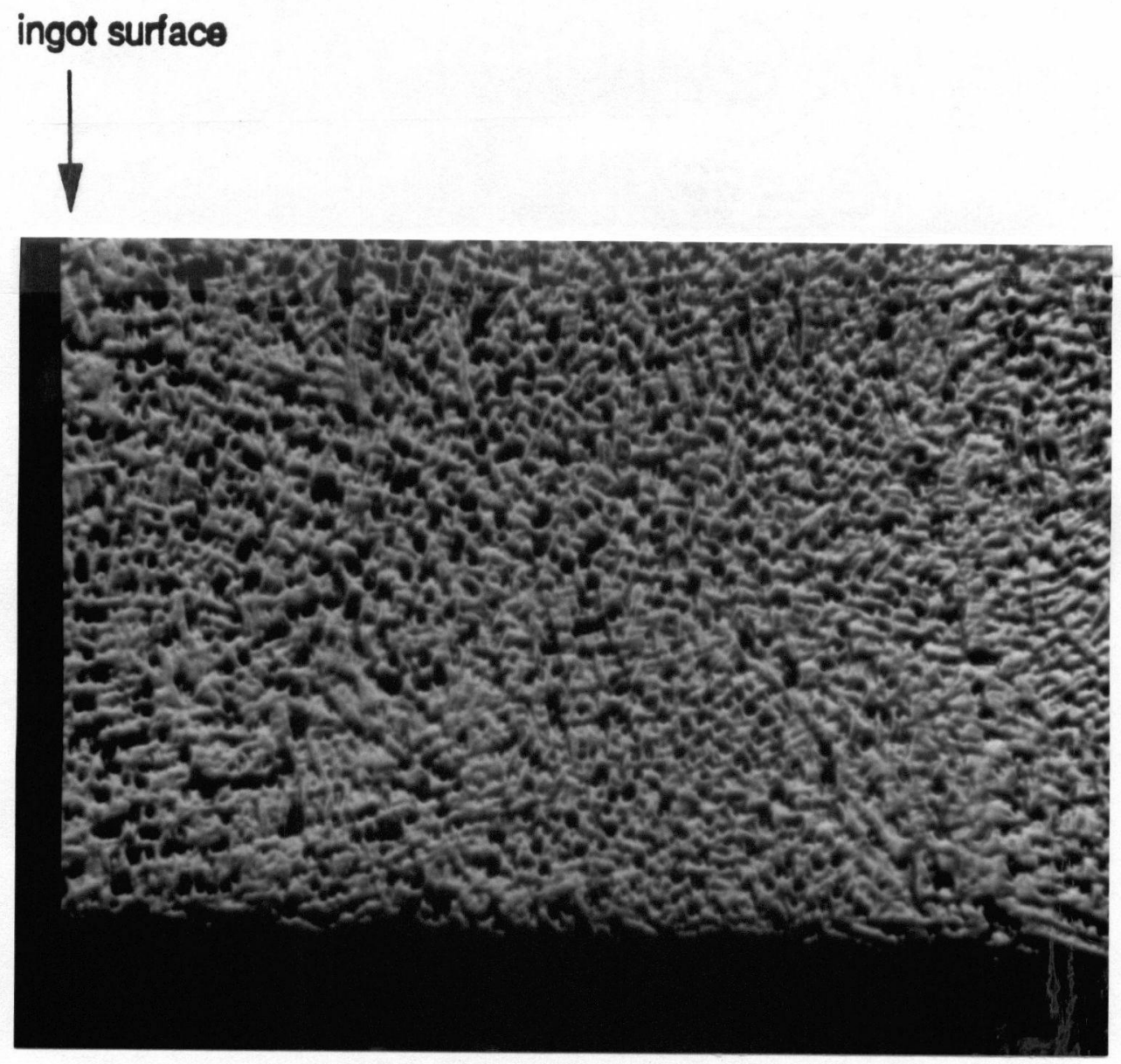

ingot radial direction

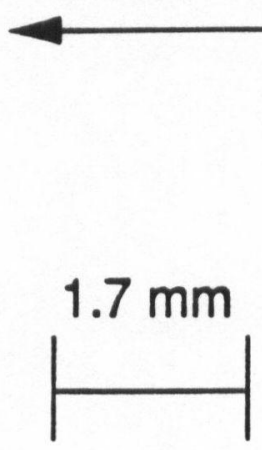

Figure 4.54: Microphotograph of a cross-sectional sample at the ingot surface (stirred zone). 


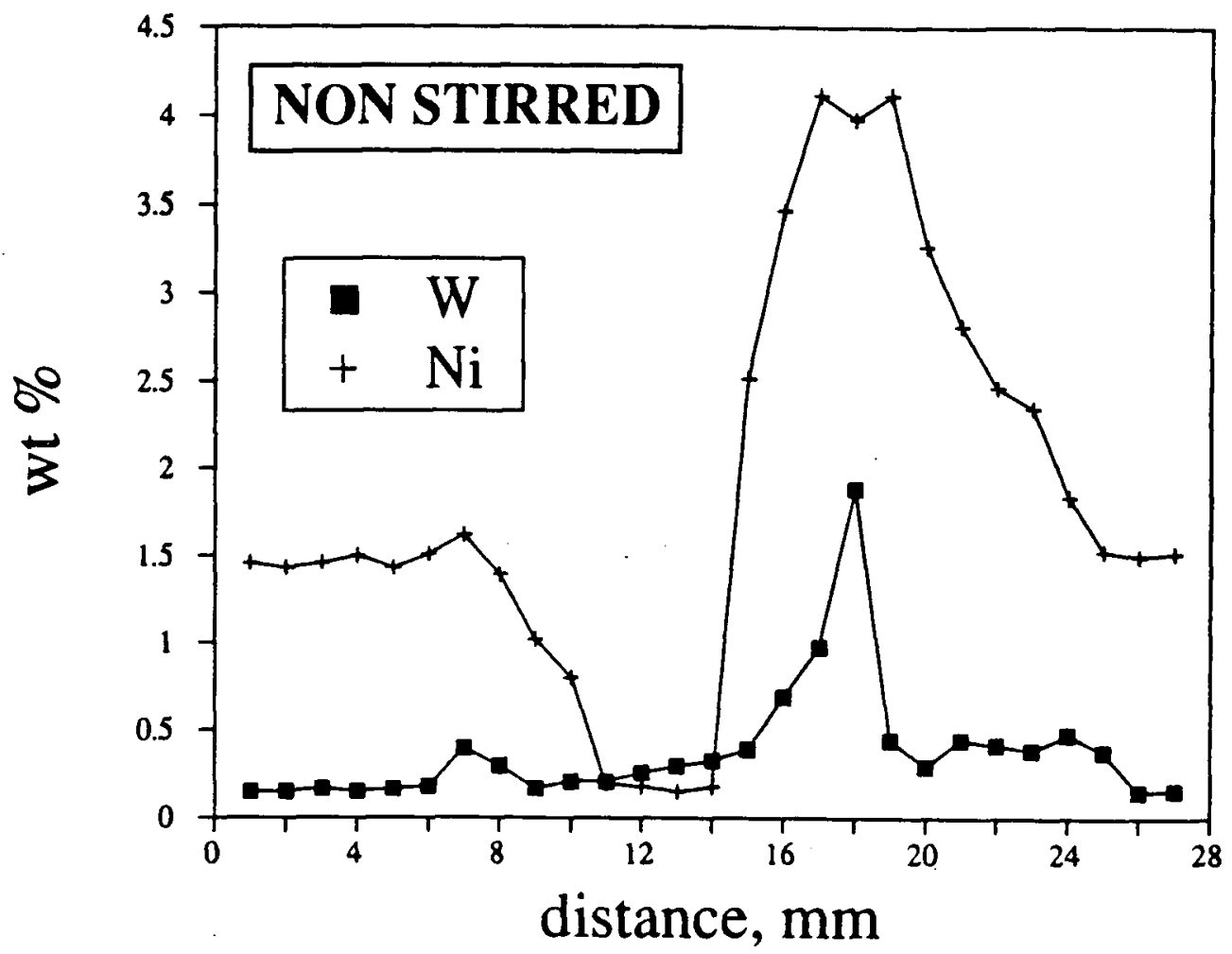

Figure 4.55: Nickel and tungsten concentration along the longitudinal axis. Ingot XI. 


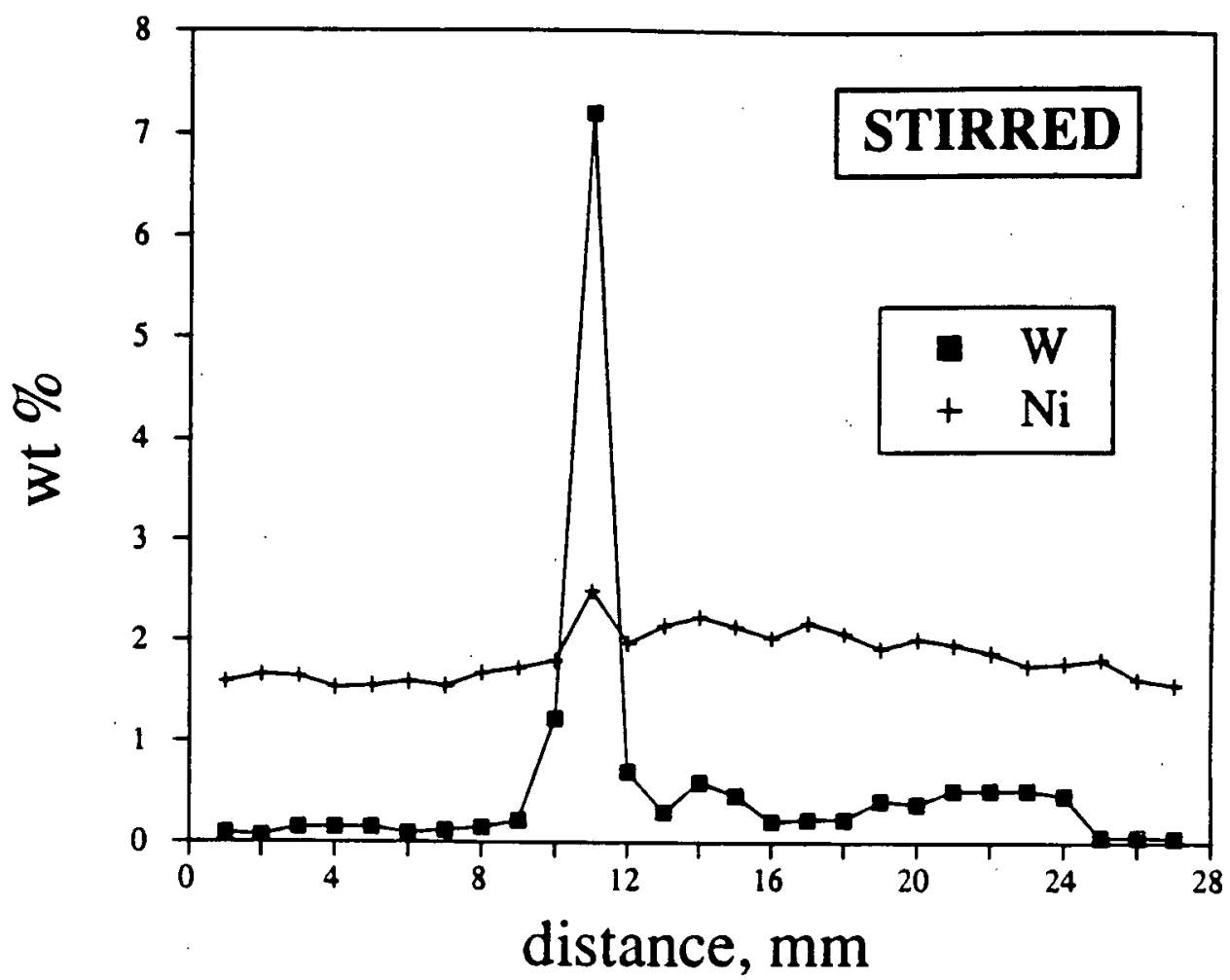

Figure 4.56: Nickel and tungsten concentration along the longitudinal axis. Ingot XII. 


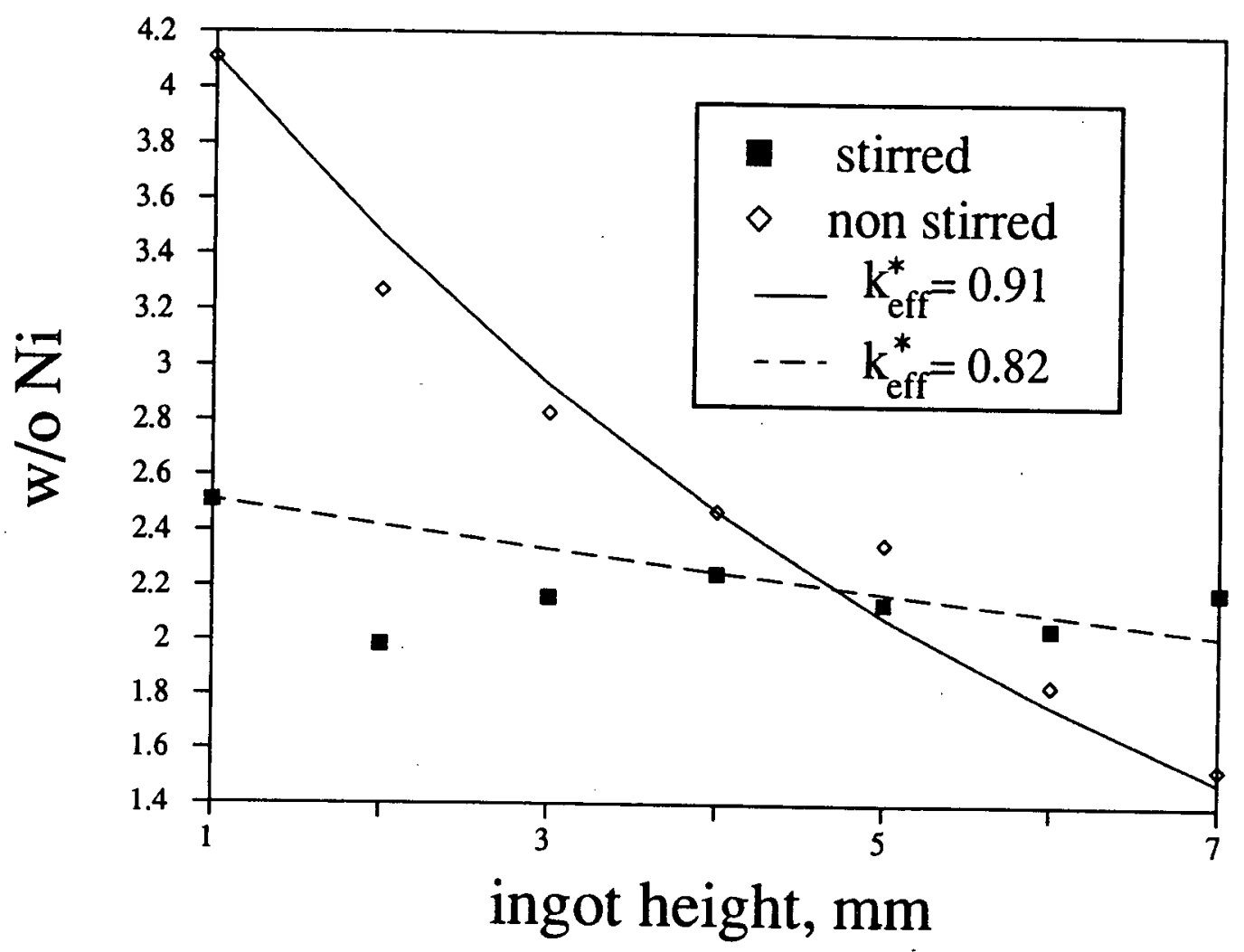

Figure 4.57: Effective distribution coefficient for experiments with and without stirring. 


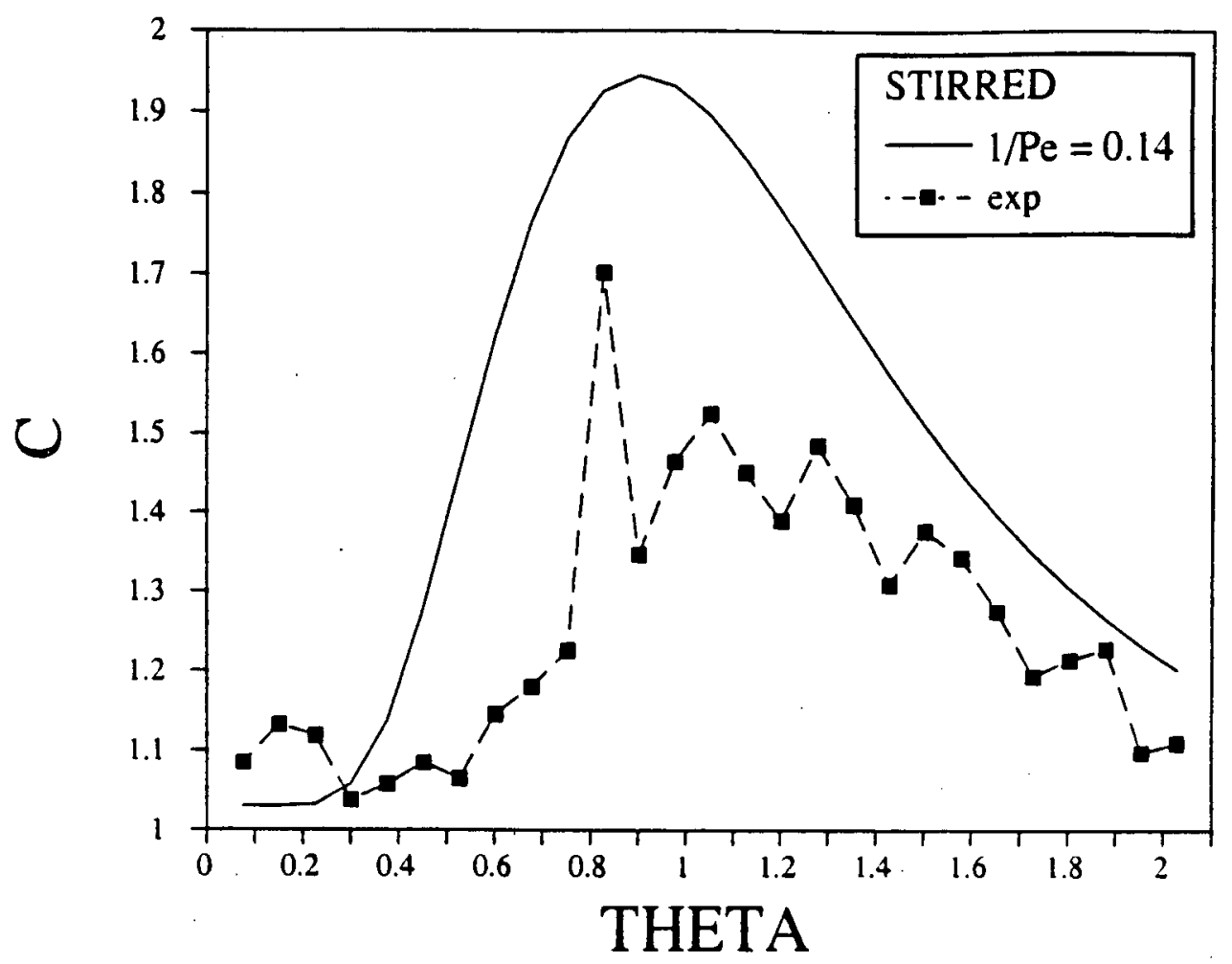

Figure 4.58: Dispersion number for experiment XII (with stirring). 


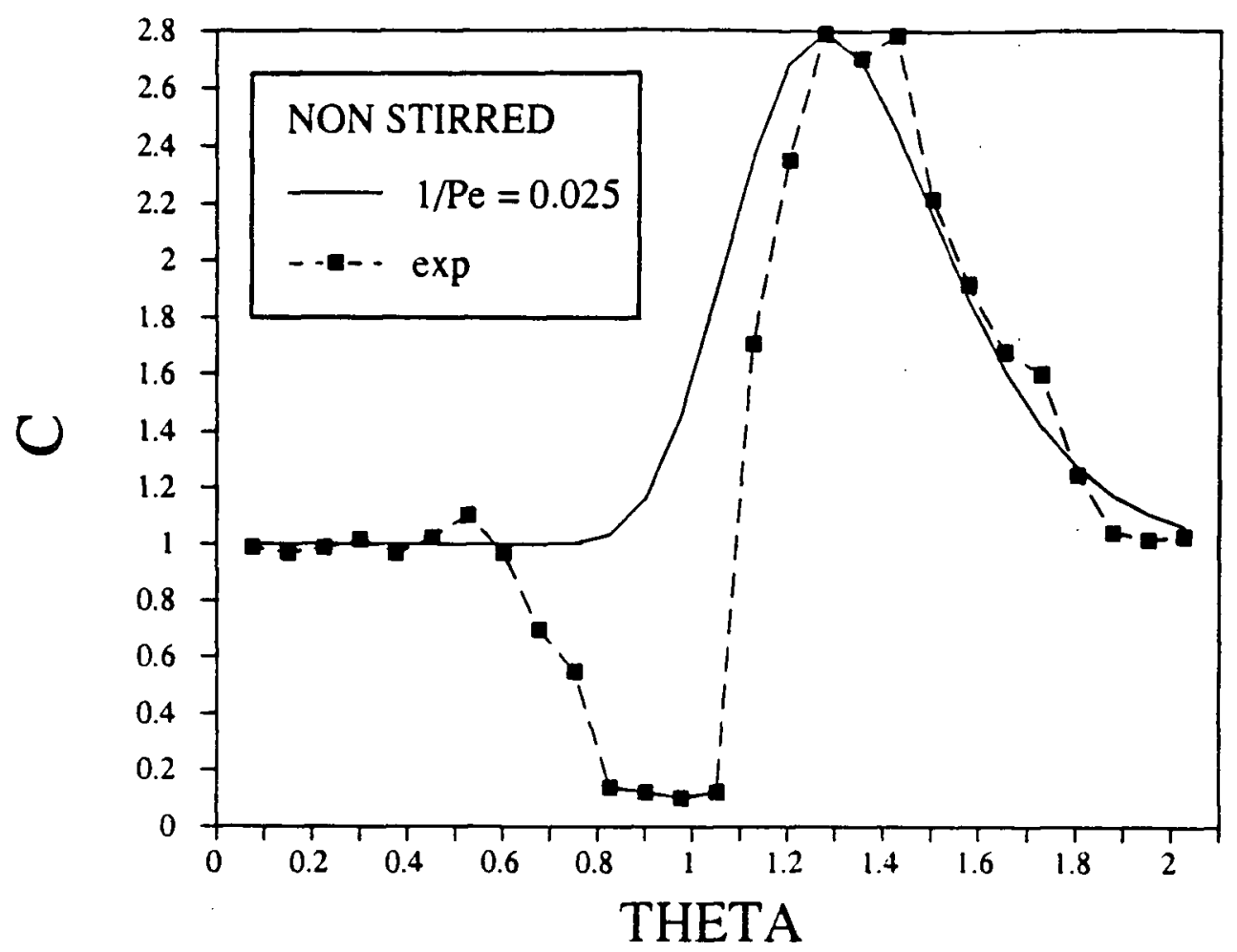

Figure 4.59: Dispersion number for experiment XI (without stirring). 


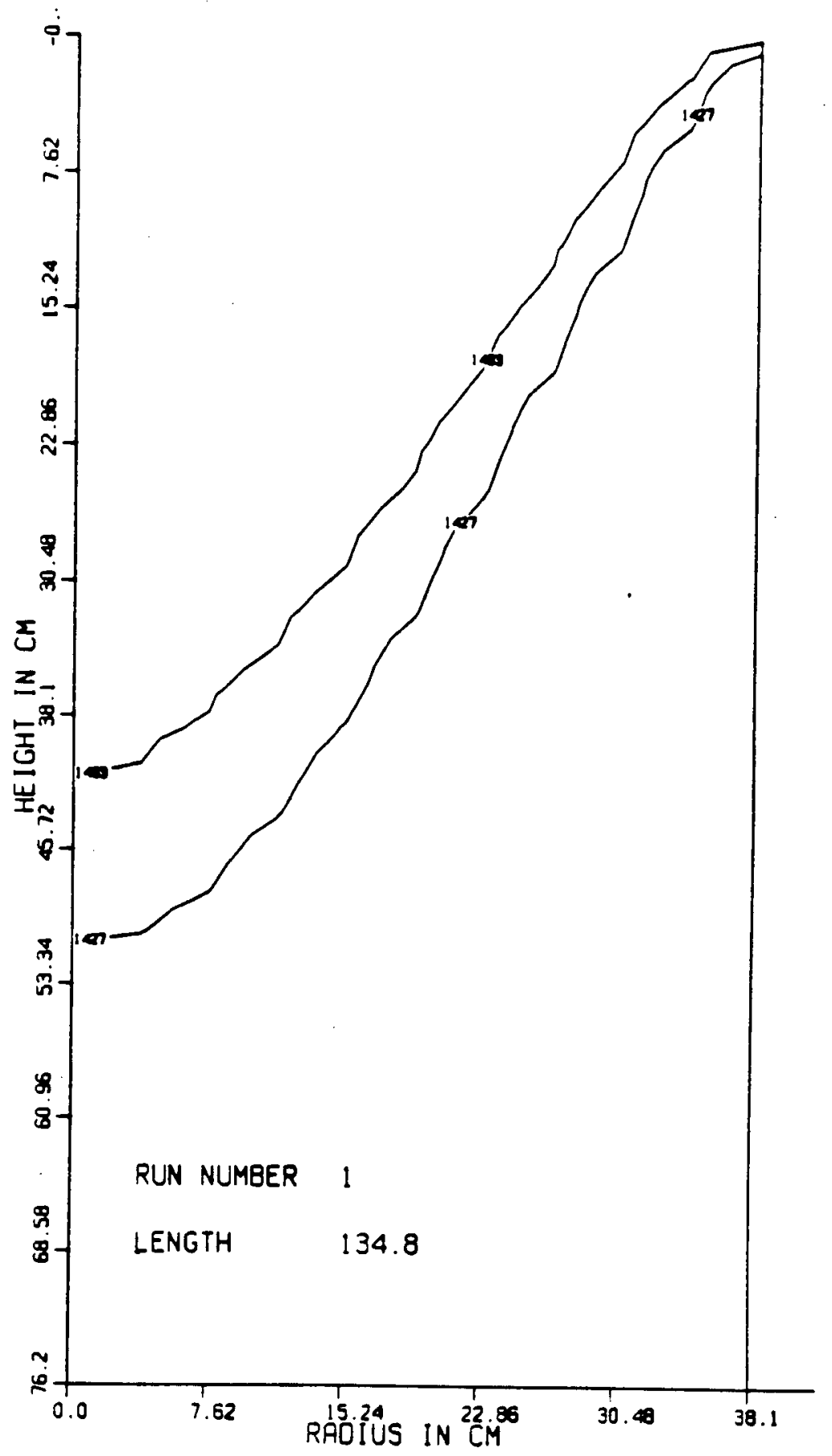

Figure 4.60: Pool profile for a hypothetical 4340 alloy steel ingot. 


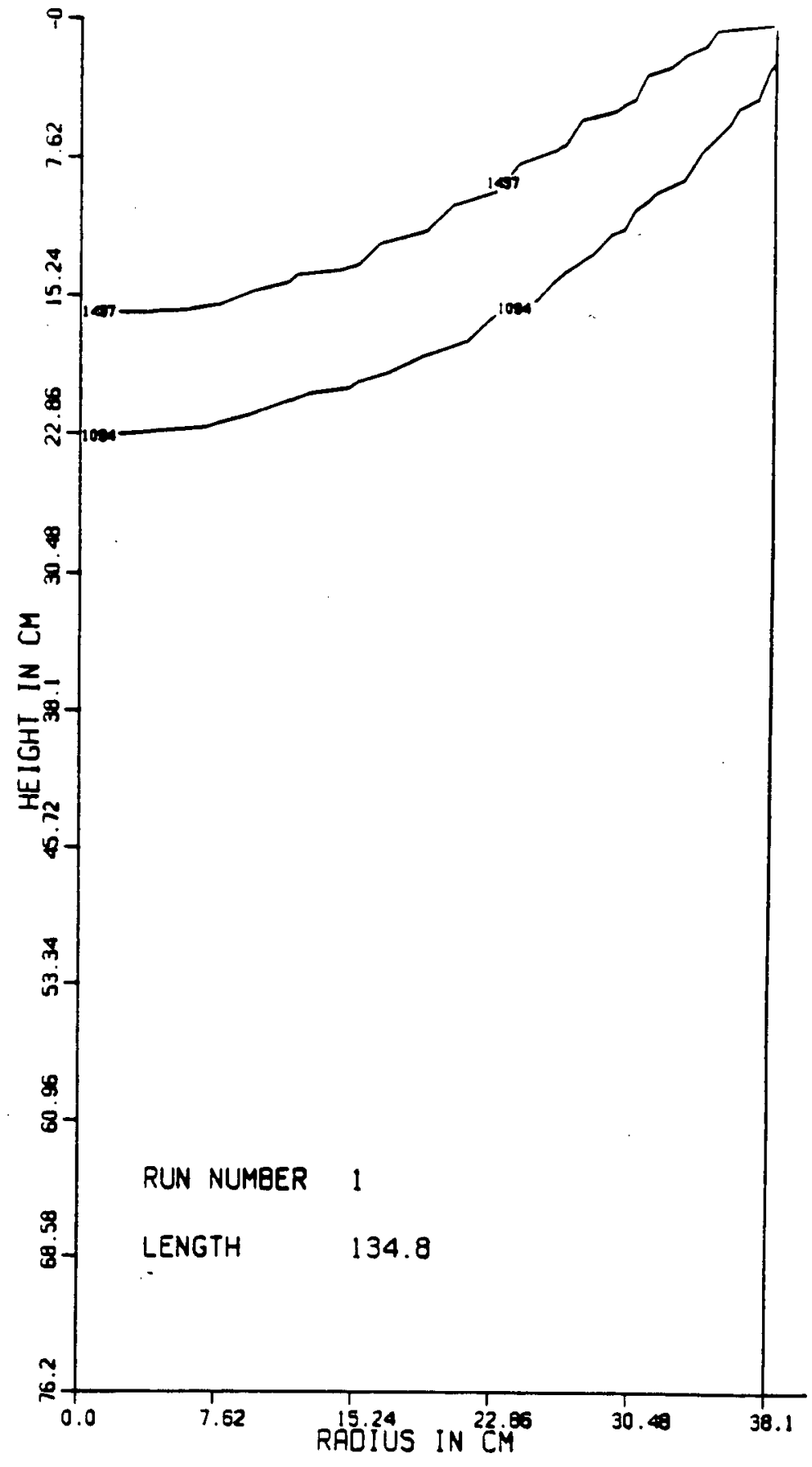

Figure 4.61: Pool profile for a hypothetical $\mathrm{Cu}-50 \% \mathrm{Fe}$ ingot. 


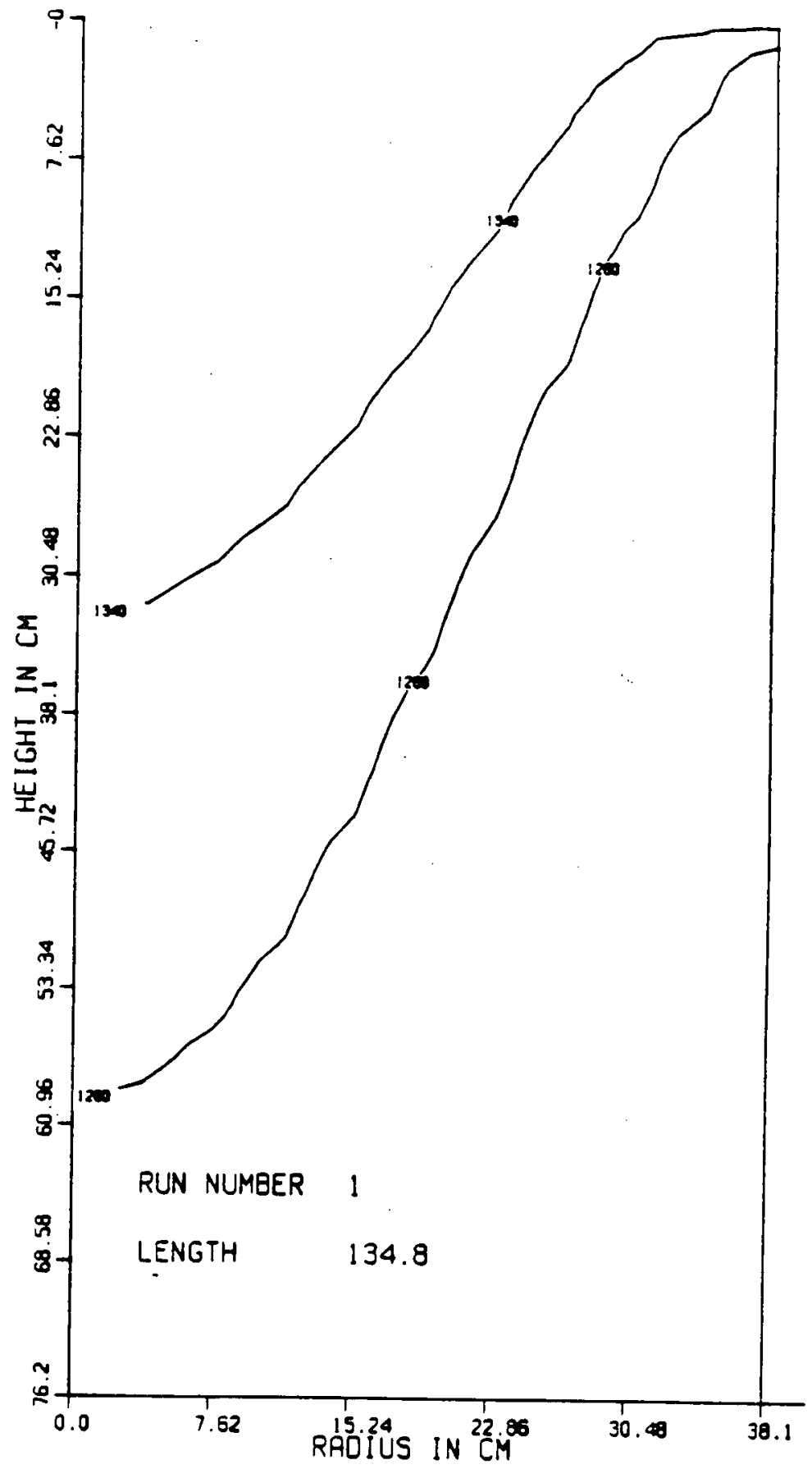

Figure 4.62: Pool profile for a hypothetical Inconel 718 ingot. 


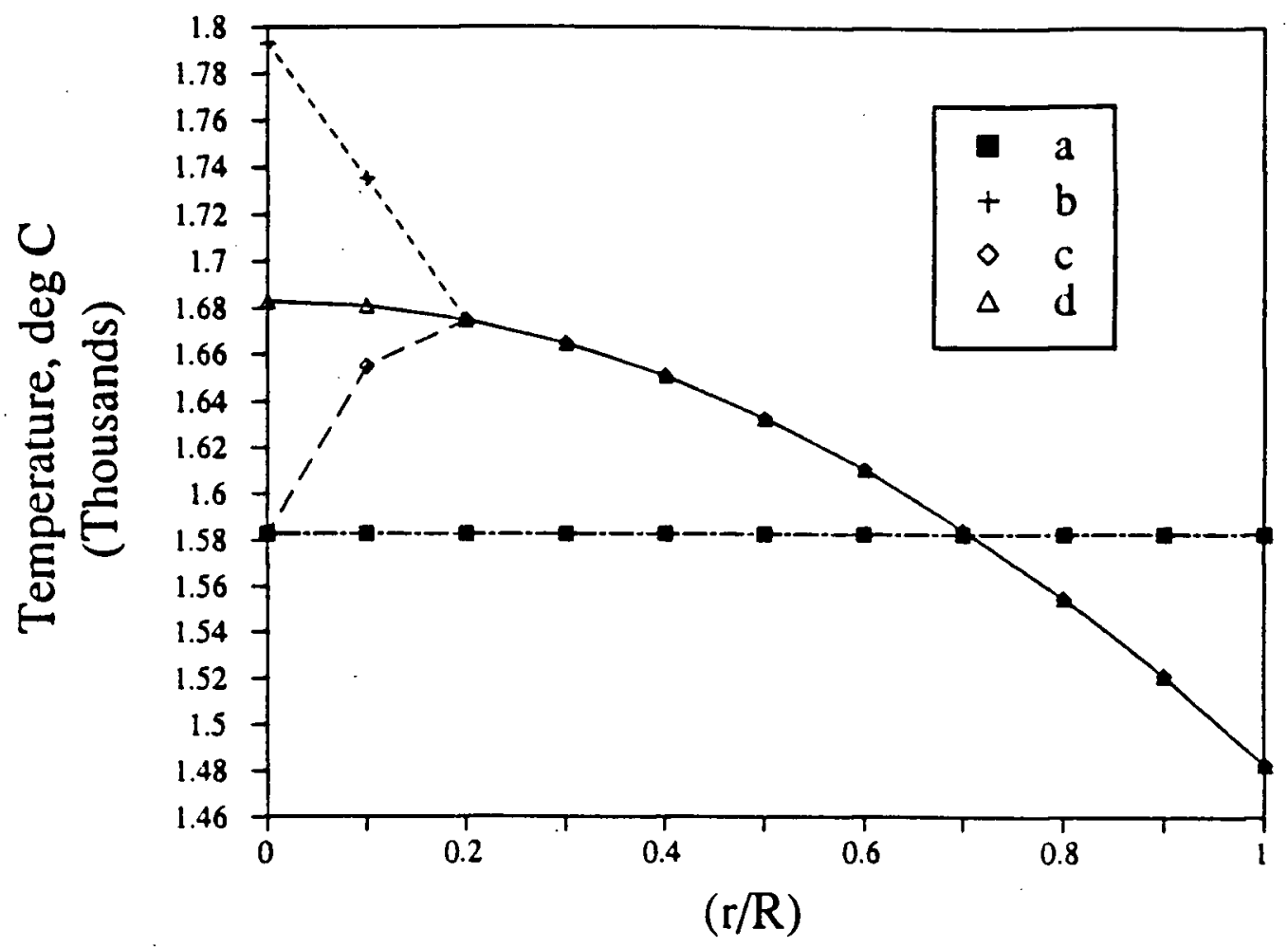

Figure 4.63: Top temperature distributions used in the study of its effect on the thermal field. Curve (d) represents the distribution used throughout the rest of the calculations. 


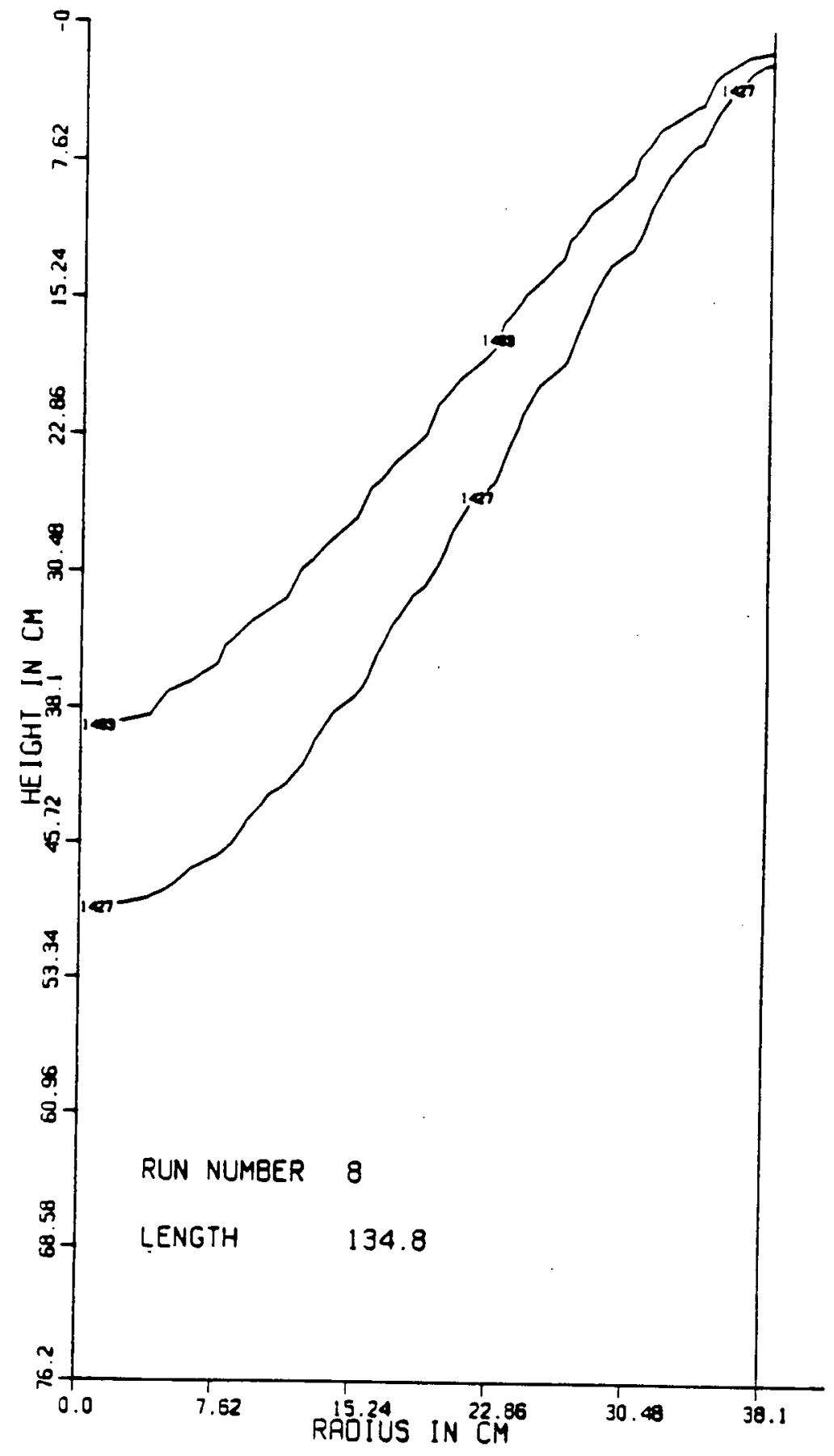

Figure 4.64: Pool profile resulting when curve (a) in fig. 4.63 is used. 


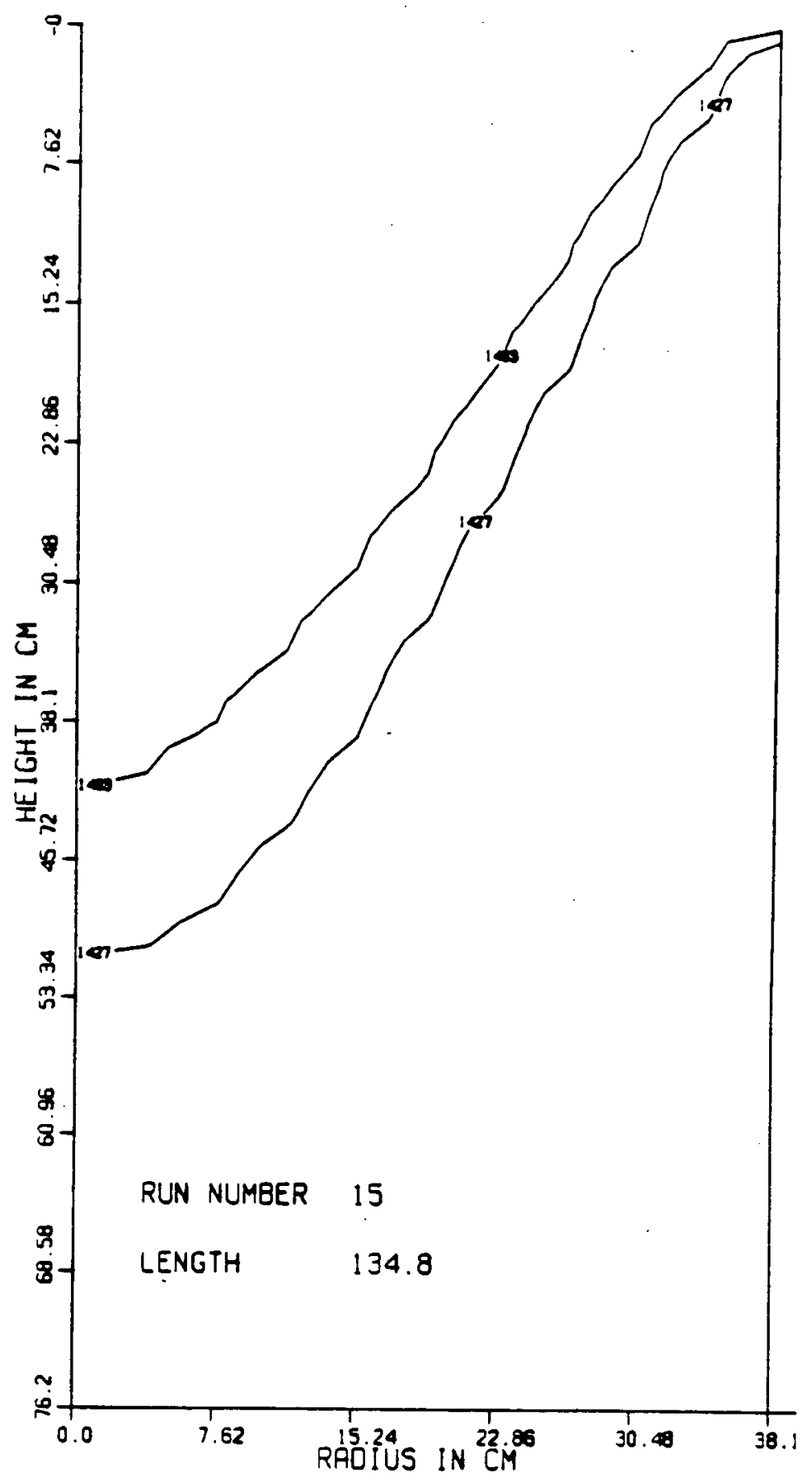

Figure 4.65: Pool profile resulting when curve (b) in fig. 4.63 is used. 


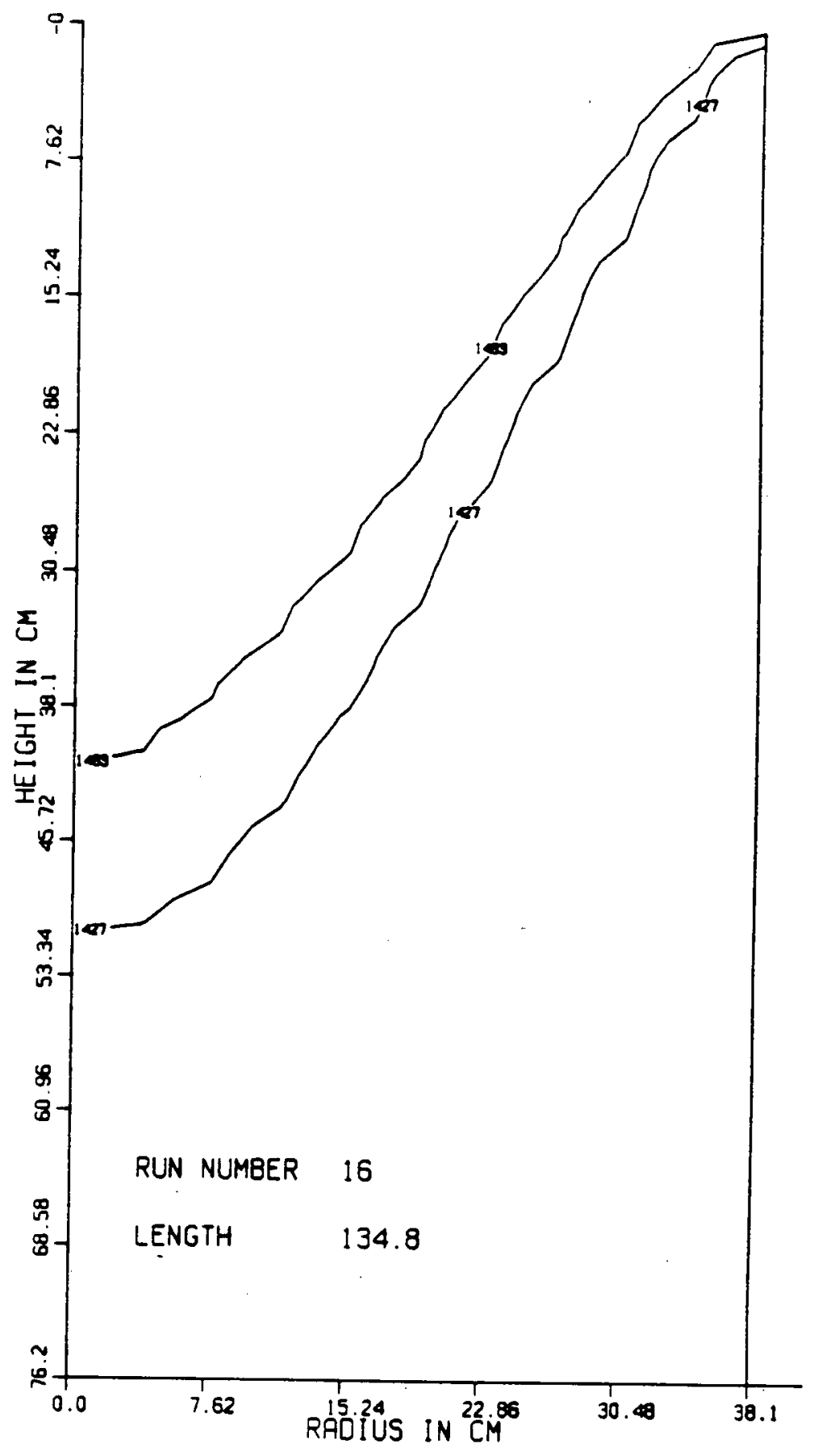

Figure 4.66: Pool profile resulting when curve (c) in fig. 4.63 is used. 


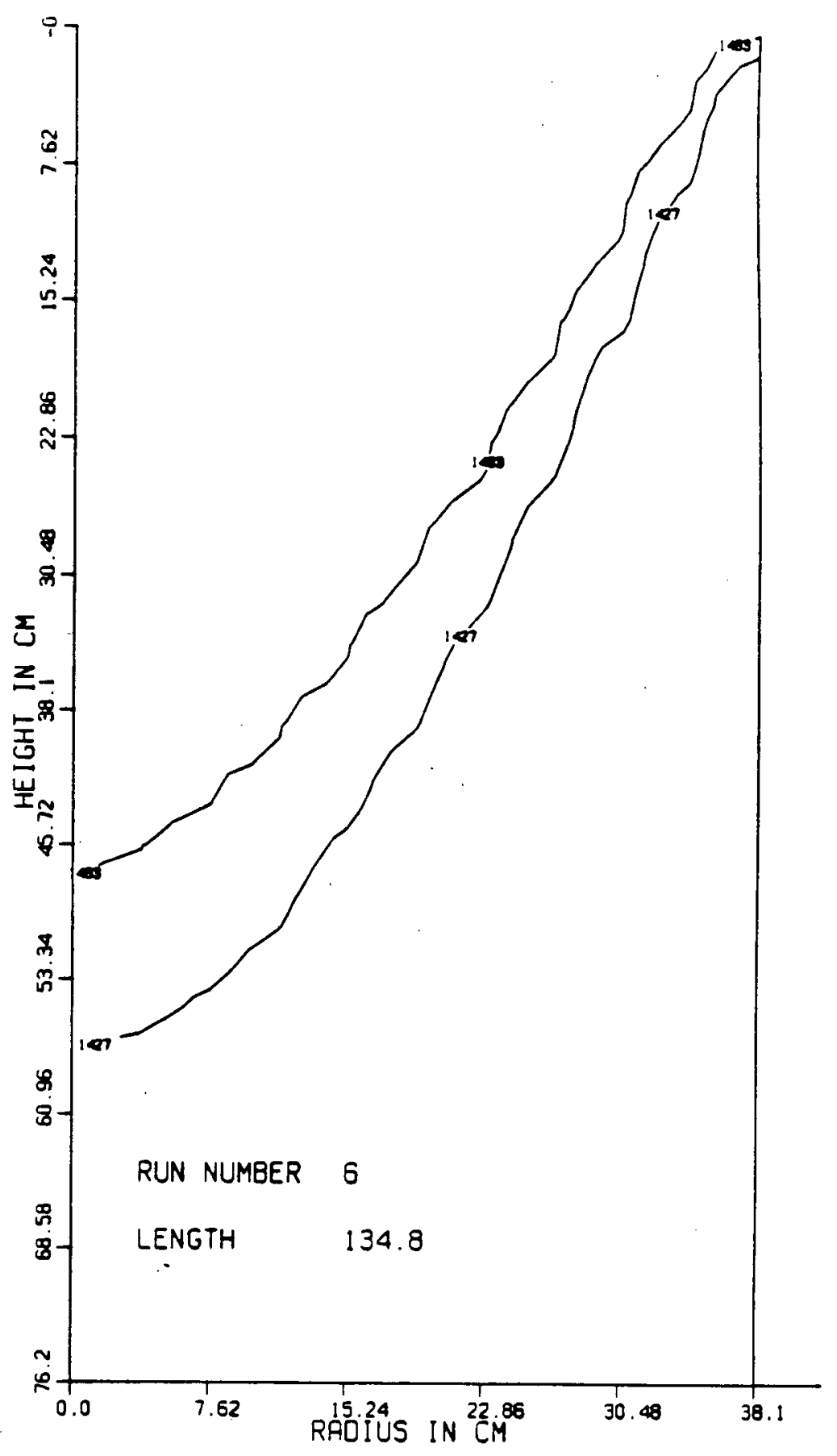

Figure 4.67: Pool profile resulting when an isotropic $k_{\text {eff }}$ equivalent to $5 \times k_{\text {at }}$ is used. 


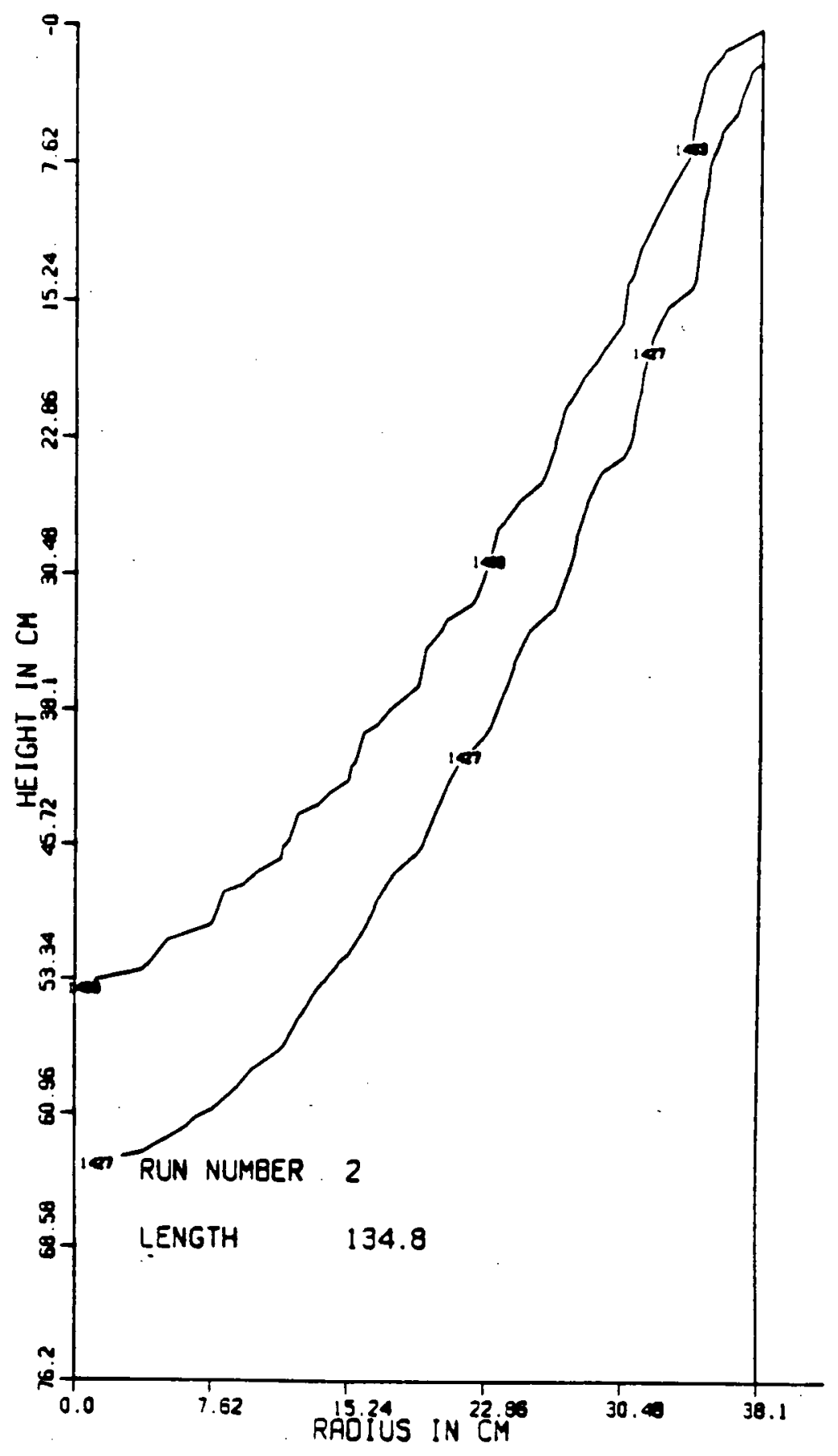

Figure 4.68: Pool profile resulting when an isotropic $k_{\text {eff }}$ equivalent to $10 \times k_{\text {at }}$ is used. 


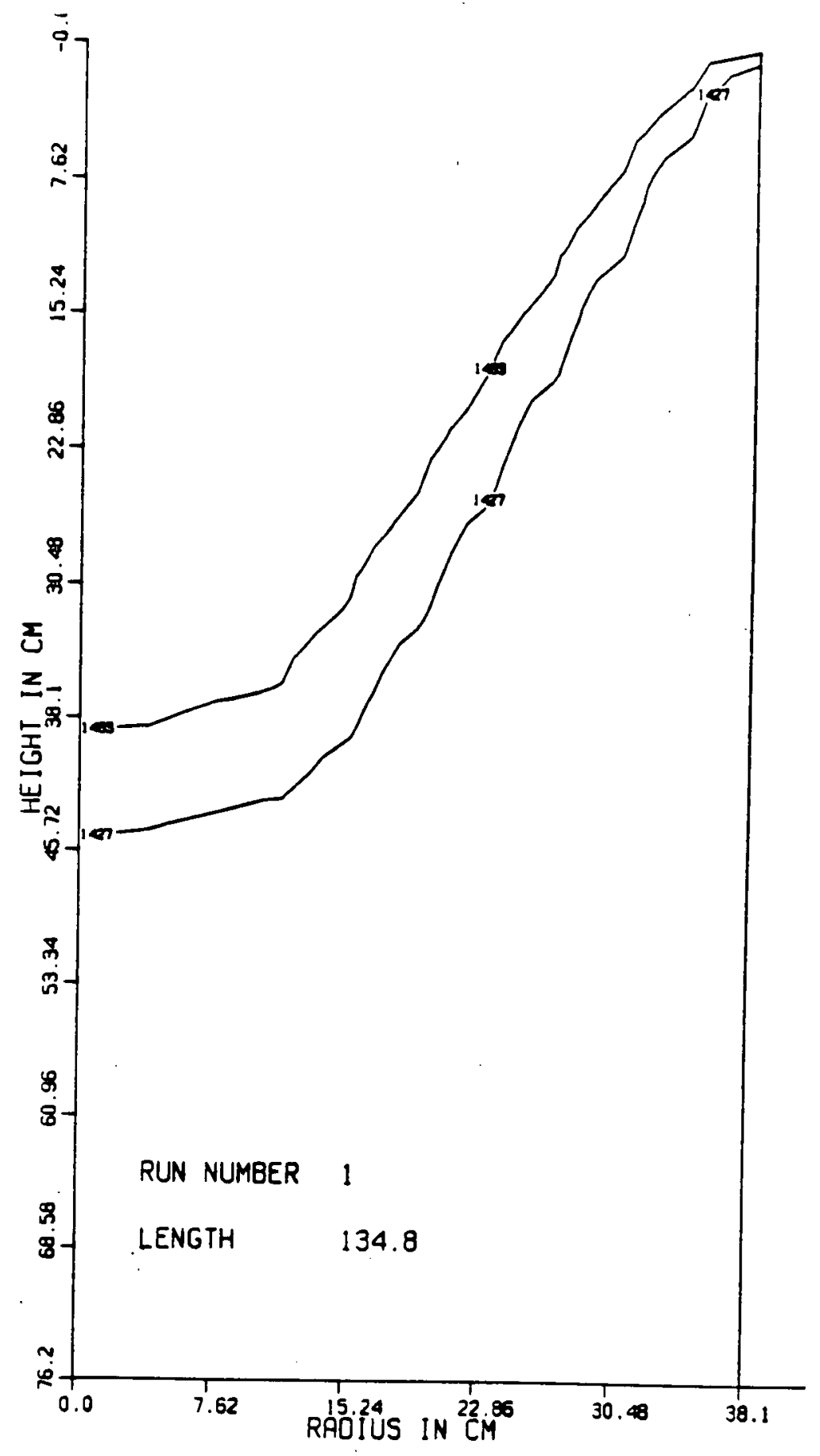

Figure 4.69: Pool profile resulting when an anisotropic $k_{\text {eff }}$ is used. The ratio $k_{\text {eff }}^{\mathbf{z}} / k_{\text {eff }}^{\mathbf{r}}$ is 5.0. 


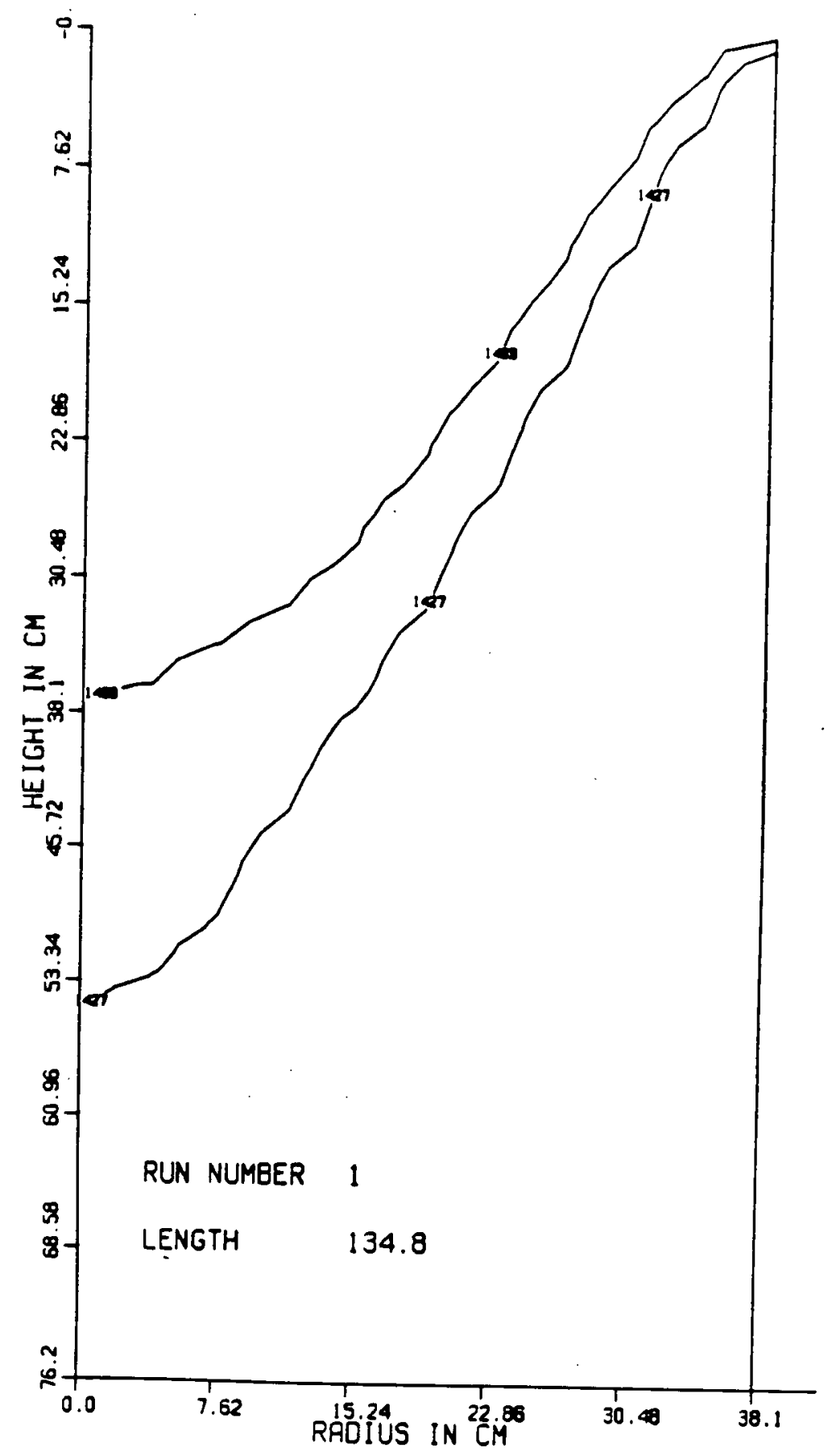

Figure 4.70: Pool profile resulting when an anisotropic $k_{\text {eff }}$ is used. The ratio $k_{\text {eff }}^{z} / k_{\text {eff }}^{r}$ is 0.2 . 


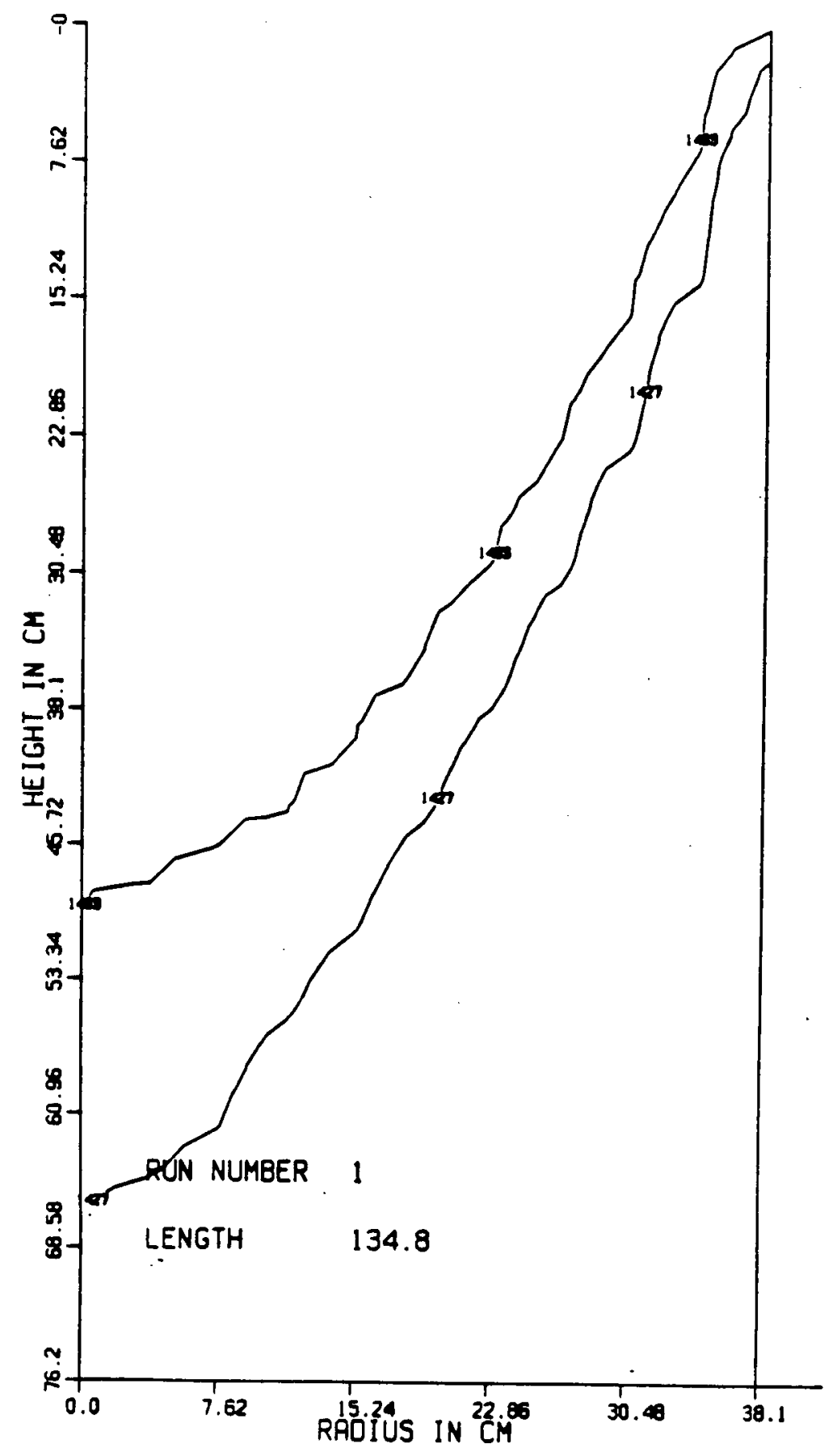

Figure 4.71: Same as fig. 4.70 except that $k_{\text {eff }}$ has been increased to 5.0. 


\section{Chapter 5}

\section{Summary and recomendations for future work}

\subsection{Summary}

During the course of the present work a stirring device based on the application of an $\mathrm{AC}$ electromagnetic field during the AC operation of a laboratory-scale ESR furnace was designed and built. The electromagnetic field damping caused by the aluminium moulds used in conventional ESR practice was overcome by substituting parts of the mould with graphite.

The externally applied field caused a vigorous rotation of the slag. The metallurgical effects were investigated via metallographic analysis. The results show a more pronounced effect on the solidification structure as the exciting current increases. It is apparent that the solidification front does not immediatly 'see' the change in the thermal field produced by the stirrer which suggests that the flow in the bulk of the liquid does not penetrate deep enough into the interdendritic region. In both, steady- and unsteady-sate experiments a thin segregated band accompanied the start of stirring. Also, the pool shape has an important influence on the effects caused by EMS.

The mixing characteristics of the liquid pool were studied using a chemical tracer. Both an effective distribution coefficient and the reactor dispersion number were obtained for experiments with and without stirring. The fitted parameters indicate a larger degree of mixing when stirring was applied.

The resulting electromagnetic body force was modelled using the mutual inductance 
technique. It was found that this force is mainly confined to the skin depth : at the mould wall and at the liquid metal-slag interphase. A previously developed code was used to simulate the thermal regime. The steady-state code was modified to include an effective thermal conductivity with vectorial properties. Even with this modification it was not possible to reproduce the experimental pool profiles.

\subsection{Recommendations for future work}

In the present investigation the mould arrangment was composed by three moulds of different diameters. It is thought that a single mould with carefully machined segments filled with a nonconductive, refractory material is required. A comprehensive experimental programme for quantifying the segregation levels resulting from EMS during production of alloys of practical interest would be advisable. Also, the mechanical properties of stirred ingots should be obtained.

The model used to estimate the electromagnetic body force was greatly simplified and not verified. A more detailed model accompanied by experimental verification, probably using a liquid metal or alloy of low melting point, is desirable. Regarding the thermal model, a two-dimensional aproach does not seem to be entirely adequate and therefore a three-dimensional model is recommended. Before any effort in this direction is started a careful definition of the current path is of outmost importance.

\subsection{Concluding remarks}

This work is the first step towards a more adequate understanding on the effects of applying AC electromagnetic fields during ESR. Much more work has to be done in both the metallurgical consequences of EMS and the design of appropriate stirring devices before this technology can be safely applied in an industrial environment. 


\section{Bibliography}

[1] G. Hoyle. 'Electroslag processes'. Applied Science Publishers. London, 1983.

[2] 'The Electroslag Melting Process'. U.S. Bureau of Mines, Bulletin No. 669, 1976.

[3] E. Plockinger. J. Iron and Steel Inst., 211, (8), 1973, pp. 533-541.

[4] H.J. Klein, B.H. Rosof, C.L. Jeanfils and J.H. Chen. 'The Argon Oxygen Decarburization and Electroslag Remelting Processes'. In 'Metallurgical Treatises', The Metallurgical Society of AIME, Warrendale, Pa., 1981.

[5] 'Electroslag Remelting and Plasma Arc Melting'. Report of the Comitte on Electroslag Remelting and Plasma Arc Melting. National Academy of Sciences. Washington, D.C., 1976.

[6] A. Mitchell and R.M. Smailer. Int. Met. Rev., Nos. 5-6, 1979. pp. 231-264.

[7] Ibid., p. 231.

[8] R. Schlatter. J. of Metals. May 1972, pp. 17-25.

[9] F.N. Darmara. J. of Metals. Dec. 1967, pp. 42-48.

[10] W. H. Sutton. Proc. of the 7th Int. Conf. on Vacuum Metallurgy, Special Meltings and Metallurgical Coatings. Nov. 26-30, 1982. Tokyo. The Iron and Steel Institute of Japan. Pp. 904-915 
[11] J.D. Lavers and P.P. Biringer. Proc. of Symp. Metallurgical Applications of Magnetohydrodynamics. Sept. 6-10, 1982. Cambridge. Ed. by H.K. Moffat and M.R.E. Proctor. The Metals Society. 1984. Pp. 62-78.

[12] J. Szekely and C.W. Chang. Proc. of the 1977 Vacuum Metallurgy Conf. June 20-22, 1977. Ed. by R. C. Krutenat. Science Press. Princeton, 1977. Pp. 15-48.

[13] D. Schieber. 'Electromagnetic Induction Phenomena' in 'Springer Series in Electrophysiscs'. Vol. 16. Ed. by T. Tamir. Springer-Verlag. Berlin, 1986.

[14] V.C.A. Ferraro and C. Plumpton. 'An Introduction to Magnetofluid Mechanics'. Clarendon Press. Oxford, 1966.

[15] Y.R. Fautrelle. J. Fluid Mech., 102, 1981, pp. 405-430.

[16] J. A. Shercliff. Metals Technology. Feb. 1984, vol. 11, pp. 71-76.

[17] J. A. Shercliff. J. Fluid Mechanics, 40, 1970, pp. 241-250.

[18] R. Moreau. Proc. of 2nd Beer-Sheva Int. Seminar on MHD Flows and Turbulence. 1978. Ben Gurion University of the Negev, Beer-Sheva, Israel. Ed. by H. Branover. Israel Univ. Press, 1980. Pp. 65-82.

[19] J.L. Meyer, F. Durand, R. Ricou and C. Vivés . Met. Trans. B, 15B, Sept. 1984, pp. 471-478.

[20] J.L. Meyer, N. El-Kaddah, J. Szekely, C. Vivés . and R. Ricou. Met. Trans. B, 18B, Sept. 1987 , pp. 529-538.

[21] Ch. Vivés and R. Ricou. Met. Trans. B, 16B, June 1985, pp. 227-235. 
[22] Ch. Vivés and R. Ricou. Proc. of Symp. Metallurgical Applications of Magnetohydrodynamics. Sept. 6-10, 1982. Cambridge. Ed. by H.K. Moffat and M.R.E. Proctor. The Metals Society. 1984. Pp. 24-32.

[23] E.D. Tarapore, J.W. Evans and J. Langfeldt. Met. Trans. B, 8B March 1977, pp. 179-184.

[24] J. Szekely, H. Choudhary and S. Woodruff. Proc. of 39th Electric Furnace Conf.. Dec. 8-11, 1981. Houston. The Electric Furnace Div., The Iron and Steel Society of AIME. pp. 72-82.

[25] J. Szekely, C. W. Chang and R.E. Ryan. Met. Trans. B, 8B, June 1977, pp. 333-338.

[26] D.J. Moore and J.C.R. Hunt. Proc. of 3rd Beer-Sheva Int. Seminar on MHD Flows and Turbulence. 1981. Ben Gurion University of the Negev, Beer-Sheva, Israel. John Wiley \& sons.

[27] D.R. Frankl. 'Electromagnetic theory'. Prentice-Hall, Inc. New Jersey, 1986, p. 268.

[28] E. Taberlet and Y.R. Fautrelle. Proc. of 4th Beer-Sheva Int. Seminar on MHD Flows and Turbulence. Feb. 27- March 2, 1984. Ben Gurion University of the Negev, Beer-Sheva, Israel. Ed. by H. Branover, P.S. Lykoudis and M. Mond. The American Institute of Aeronautics and Astronautics. 1985. Pp. 680-693.

[29] Proc. of the Conf. Ligth Metals 1985. Feb. 24-28, 1985. New York. Ed. by H.O. Bohner. The Metallurgical Society of AIME. Pp. 593-605.

[30] J.C. Weber. Continuous casting: Melting-Casting-Control. Pp. 123-138.

[31] D.E. Tyler. Continuous casting: Melting-Casting-Control. Pp. 115-122. 
[32] R. Sautebin and W. Haller. Proc. of the Conf. Ligth Metals 1985. Feb. 24-28, 1985. New York. Ed. by H.O. Bohner. The Metallurgical Society of AIME. Pp. 1301-1308.

[33] Y. Shirota, K. Katohgi, K. Klein, H.-J. Engell and D. Janke. Trans. ISIJ, 1985, vol. 25, pp. $1132-1140$.

[34] R.I. Asfahani, D.S. Shahapurkar, Y.V. Murty, J.A. Patchett and G.J. Abbaschian. J. of Metals, April 1985, pp. 22-26.

[35] N. El-Kaddah and J. Szekely. Met. Trans. B, 14B, Sept. 1983, pp. 401-410.

[36] A. Kaye and A. Street. 'Die Casting Metallurgy'. Butterworth Scientific. London, 1982.

[37] D.F. Allsop and D. Kennedy. 'Pressure Diecasting. Part 2: The Technology of the Casting and the Die'. Pergamon Press. Oxford, 1983.

[38] D.V. Neff. Proc. of the Conf. Aluminium Technology '86. March 11-13, 1986. London. Ed. by T. Sheppard. The Institue of Metals, 1986. Pp. 739-746.

[39] P.J. Bamji and F.W. Pierson. Proc. of the Conf. Ligth Metals 1983. March 6-10, 1983. Atlanta, GA. The Metallurgical Society of AIME. Pp. 953-961.

[40] J.K. Farmer and R. Hans. Proc. of the Conf. Die Casting Technology - Yesterday's Art, Today's Science. Oct. 31- Nov. 3, 1983. Minneapolis, Minn. Society of Die Casting Engineers. 1983. Pp. 6.

[41] 'Taking the Heat off Molten Metal Handling. Part I : Nonferrous'. Ed. by D.P. Kamichi.Cover story in Modern Casting. Oct. 1984, pp. 22-24.

[42] P.J.F. Bamji and N.P.F. Fitzpatrick. Patent No. EPO 129975 (EUR), 17 May, 1984. 
[43] A.W. Jones. Proc. of the Conf. Aluminium Technology '86. March 11-13, 1986. London. Ed. by T. Sheppard. The Institue of Metals, 1986. Pp. 763-770.

[44] J.P. Birat and J. Choné. Ironmaking and Steelmaking, 1983, vol. 10, No. 6, pp. 269-281.

[45] Y. Sundberg. Proc. of Symp. Metallurgical Applications of Magnetohydrodynamics. Sept. 6-10, 1982. Cambridge. Ed. by H.K. Moffat and M.R.E. Proctor. The Metals Society. 1984. Pp. 217-223.

[46] D.E. Cooper and R. J. Krieger. Proc. of the Conf. Arcs and Inert Atmospheres in Vacuum. April 30 - may 1, 1956. San Francisco, CA. Ed. by W.E. Kuhn. J. Wiley \& Sons. 1956. Pp. 57-68.

[47] D.R. Carnahan and J.H. Kelley. Trans. of Vacuum Metallurgy Conf. 1959. June 1-3, 1959. Ed. by R.F. Bunshah. New York University Press. 1960. Pp. 49-57.

[48] M.A. Keane. Ph.D. Thesis. MIT, 1973.

[49] G.J. Davies. Solidification and Casting. Applied Science Publishers LTD. London, 1973 , p. 117.

[50] P.N. Hansen and J.T. Berry. Proc. of the Conf. on Modeling of Casting and Welding Processes. Aug. 3-8, 1980. Ridge, N.H. Ed. by H.D. Brody and D. Apelian. The Metallurgical Society of AIME. Pp. 497-502.

[51] T.S. Piwonka. Ibid. , pp. 503-518.

[52] L.J.D. Sully. Ibid. , pp. 509-512.

[53] M.C. Flemings. Ibid. , pp. 533-548. 
[54] V. Pasarica and P. Marcu. Cercet. Metal., 1975, 16, pp. 575-599.

[55] V. Pasarica, E. Georgescu and A. Benescu. Metalurgia, July 1983, 35, (7), pp. 308312.

[56] R.J. Mc Donald and J.D. Hunt. TMS-AIME,(245), 1969, pp. 1993-1997.

[57] S. Asai and I. Muchi. Trans. ISIJ, 1978, vol. 18, pp. 90-98.

[58] K.A. Jackson and J.D. Hunt. Acta Met., 13, 1965, pp. 1212-1215.

[59] C. Lemaignan, D. Camel and J. Pelissier. Mem. Etud. Sci. Rev. Metall., Dec. 1981, 78, (12), pp. 663-667.

[60] A.S. Ballantyne and A. Mitchell. Proc. of Conf. Solidification and Casting of Metals. July 18-21, 1977. Sheffield, England. The Metals Society, 1979. Pp. 363-370.

[61] J.M. Walsh and B.H. Kear. Met. Trans. A, 6A, Jan. 1975, pp.226-229.

[62] J.D. Verhoeven. Fundamentals of physical metallurgy. John Wiley \& Sons, New York, 1975, p. 245.

[63] B.A. Rickinson and D.H. Kirkwood. Proc. of Conf. Solidification and Casting of Metals. July 18-21, 1977. Sheffield, England. The Metals Society, 1979. Pp. 44-52.

[64] T.F. Bower, H.D. Brody, and M.C. Flemings. Trans. TMS-AIME,(236), may 1966, PP. 624-634.

[65] J.A. Spittle and D.M. LLoyd. Proc. of Conf. Solidification and Casting of Metals. July 18-21, 1977. Sheffield, England. The Metals Society, 1979. Pp. 15-20.

[66] T. Okamoto. J. Cryst. Growth, 29, 1975, pp. 131-136. 
[67] M.C. Flemings, D.R. Poirier, R.V. Barone and H.D. Brody. J. Iron Steel Inst., (208), 1970, pp. 371-381.

[68] T. Okamoto. J. Cryst. Growth, (29), 1975, pp. 137-146.

[69] T.Z. Kattamis and M.C. Flemings. Trans. TMS-AIME, (233), May 1965, pp. 992999.

[70] B. Chalmers. 'Principles of Solidification'. John Wiley \& Sons. New York, 1964. p. 171.

[71] I. Ghias and W.R. Wilcox. Met. Trans. B, 12B, June 1981, pp. 249-253.

[72] P.R. Beeley. Proc. of Conf. Solidification and Casting of Metals. July 18-21, 1977. Sheffield, England. The Metals Society, 1979. Pp. 319-324.

[73] D.E. Adams. J. Inst. of Metals, vol. 75, 1948, pp. 809-838.

[74] A. Hultgren. Scand. J. Metallurgy, 2, (5), 1973, pp. 217-227.

[75] H.F. Taylor . 'Foundry Engineering', Wiley \& Sons. New York,1959, p. 206.

[76] A.F. Giamei and B.H. Kear. Met. Trans., 1, 1970, pp. 2185-2192.

[77] K.O. Yu, J.A. Domingue, G.E. Maurer and H.D. Flanders. J. of Metals, Jan. 1986, pp. $46-50$.

[78] A. Mitchell. Ironmaking and Steelmaking, 1975, (3), pp. 198-204.

[79] R.A. Grange. Met. Trans., 2, Feb. 1971, pp. 417-426.

[80] T.F. Bower. 'Copper and its alloys' (Inst. of Metals Monograph), 1970, (34), pp. $67-73$. 
[81] M.C. Flemings and G.E. Nereo. Trans. TMS-AIME, (239), Sept. 1967, pp. 14491461.

[82] M.C. Flemings, R. Mehrabian, and G.E. Nereo. Trans. TMS-AIME, (242), Jan. 1968, pp. 41-49.

[83] M.C. Flemings and G.E. Nereo. Trans. TMS-AIME, (242), Jan. 1968, pp. 50-55.

[84] J.S. Kirkaldy and W.V. Youdelis. Trans. TMS-AIME, (212), Dec. 1958, pp. 833-840.

[85] D. J. Hebditch and J.D. Hunt. Met. Trans., 4, Aug. 1973, pp. 2008-2010.

[86] R. Mehrabian, M. Keane and M.C. Flemimgs. Met. Trans., 1, May 1970, pp. 12091220 .

[87] G.H. Geiger and D.R. Poirier. 'Transport Phenomena in Metallurgy'. AddisonWesley Publishing Co., Massachusetts, 1973. p. 91.

[88] Ibid. p. 92.

[89] I. Ohnaka and T. Fukusako. Trans. ISIJ, July 1981, 21, (7), pp. 70-77.

[90] N. Streat and F. Weinberg. Met. Trans., 5, Dec. 1974, pp. 2539-2548.

[91] B. Prabhakar and F. Weinberg. Met. Trans. B, 9B, March 1978, pp. 150-151.

[92] S. Minakawa, I.V. Samarasekera and F. Weinberg. Met. Trans. B, 16B, Sept. 1985, pp. 595-604.

[93] F. Weinberg. Met. Trans. B, 15 B, Dec. 1984, pp. 681-684.

[94] J.P. Gabathuler and F. Weinberg. Met. Trans. B, 14 B, Dec. 1983, pp. 733-741.

[95] J. Verhoeven. Met. Trans. 2, Sept. 1971, pp. 2673-2680. 
[96] T. Takahashi. The 6th Japan-USSR Joint Symp. on Physical Chemistry of Met. Processes. Tokyo, 1977, The Iron and Steel Inst. of Japan, pp. 137-148.

[97] F.J. Zanner and L.A. Bertram. SAND50-1156 Report. Sandia National Laboratories, Albuquerque, N.M., 1980.

[98] M.A. Keane. Ph. D. Thesis. MIT, 1973.

[99] A. Mitchell, R.O. Jackson and A.S. Ballantyne. Proc. of the 4th Int. Symp. on Electroslag Remelting Processes. Tokyo, June 7-8, 1973. The Iron and Steel Inst. of Japan. Pp. 69-80.

[100] S.D. Ridder, F.C. Reyes, S. Chakravorty, R. Mehrabian, J.D. Nauman, J.H. Chen and H.J. Klein. Met. Trans. B, 9B, Sept. 1978, pp. 415-425.

[101] C.L. Jeanfils, J.H. Chen and H.J. Klein. Proc. of the Conf. on Modeling of Casting and Welding Processes. Aug. 3-8, 1980. Ridge, N.H. Ed. by H.D. Brody and D. Apelian. The Metallurgical Society of AIME. Pp. 313-332.

[102] S. Kou, D. Poirier and M.C. Flemings. Met. Trans. B, 9B, Dec. 1978, pp. 711-719.

[103] D.N. Petrakis, M.C. Flemings and D.R. Poirier. Proc. of the Conf. on Modeling of Casting and Welding Processes. Aug. 3-8, 1980. Ridge, N.H. Ed. by H.D. Brody and D. Apelian. The Metallurgical Society of AIME. Pp. 285-312.

[104] S.D. Ridder, R. Mehrabian and S. Kou. Ibid. Pp. 261-284.

[105] J. Campbell. J. of Metals. July 1970, pp.23-35.

[106] H. Fredriksson and O. Jarleborg. J. of Metals, Sept. 1971, pp. 32-40.

[107] A. Samoilovich, Yu. G. Emel'yanenko and L.N. Yasnits'kii. Dop. Akad. Nauk. RSR [A], Aug. 1981, (8), 88-91. 
[108] J.D.W. Rawson, D.I. Dawson and N. Kirkhan. Proc. of the 4th Int. Symp. on Electroslag Remelting Processes. Tokyo, June 7-8, 1973. The Iron and Steel Inst. of Japan. Pp. 69-80.

[109] Y. Nakamura, N. Tokumitsu and K. Harashima,. Trans. ISIJ, 1974, 14, (3), pp. $170-175$.

[110] H. Birck and F.W. Thomas. Proc. of the 6th Int. Conf. on Special Melting. San Diego, April 23-27, 1979. American Vacuum Society, p. 820.

[111] K.C. Mills and B.J. Keene. Int. Met. Rev., 26, (1), 1981, pp. 21-69.

[112] A.H. Dilawari and J. Szekely. Met. Trans. B, 9B, March 1978, pp. 77-87.

[113] Yu. I. Zabaluev, E.I. Moshkevich, G.A. Buryakovski, G.M. Brodskii and A.A. Nikulin. Steel in USSR, Vol. 3, Aug. 1973, p. 644-645.

[114] D.A. Dudko and I.N. Rublevskii. Art. Svarka, No. 9, 1960, pp. 12-16.

[115] F. Wagner. European Concerted Action Materials for Gas Turbines Report. Sept. 1975.

[116] A.A. Nikulin, V.D. Artm'ev, L.A. Volokhowskii, M.M. Klyuev, U.V. Topilin, S.E. Volkov and A.A. Sharapov. Sb. Tr. Tsent Nauch.- Issled. Inst. Chern. Met., (75), 1970, pp. 161-167.

[117] K. Miyazawa, T. Fukaya, S. Asai, M. Choudhary and J. Szekely. Trans. ISIJ, vol. 25,1985, pp. $386-393$.

[118] S. Asai, K. Miyazawa, T. Fukaya and I. Muchi. Proc. of Symp. Metallurgical Applications of Magnetohydrodynamics. Sept. 6-10, 1982. Cambridge. Ed. by H.K. Moffat and M.R.E. Proctor. The Metals Society. 1984. Pp. 224-233. 
[119] J. Campbell and D.I. Dawson. BISRA Open Report. MG/58/77. London, 1971, 42 pp.

[120] J.F. Elliot and M. Maulvault. Proc. of the 4th Int. Symp. on Electroslag Remelting Processes. Tokyo, June 7-8, 1973. The Iron and Steel Inst. of Japan., pp. 69-80.

[121] M. Basaran, R. Mehrabian, T. Z. Kattamis and M.C. Flemings. Army Materials and Mechanics Research Center. Rep. No. CTR 74-36. April 1974.

[122] L.F. Carvajal and G.E.Geiger. Met. Trans., 2, Aug. 1976, pp. 2087-2092.

[123] Jeanfils. Proc. of the Conf. on Modeling of Casting and Welding Processes. Aug. 3-8, 1980. Ridge, N.H. Ed. by H.D. Brody and D. Apelian. The Metallurgical Society of AIME.. Pp. 261-284.

[124] B. I. Medovar, V.F. Demchenko, A.G. Burgarchenko, N.I. Tarasevich and Yu. P. Shtanko. Proc. of the 5th Int. Conf. on Vac. Met. and Electroslag Remelting Processes. Munich, Oct. 11-15, 1976. Leybold-Heraus, GmbH.. Pp. 153-156.

[125] A. S. Ballantyne and A. Mitchell. Ironmaking and Steelmaking, (4), 1977, pp. $222-$ 239.

[126] G. Sanchez Sarmiento, E. Vicente and A. Leyt. Proc. 2nd Int. Conf. on Numerical Methods in Themal Problems. Venice, Italy, July 7-10, 1981. Pineridge Press.. Pp. 697-711.

[127] A.H. Dilawari and J. Szekely. Met. Trans. B, 9B, March 1978, pp. 77-87.

[128] J. Kreyenberg and K. Schwerdtfeger. Arch. Eissenhuttenwes., 50, No. 1. Jan, 1979, pp. 1-6.

[129] M. Choudhary and J. Szekely. Met. Trans. B, 11B, Sept. 1980, pp. 439-453. 
[130] M. Choudhary and J. Szekely. Ironmaking and Steelmaking, (5), 1981, pp. 225-232.

[131] A. S. Ballantyne. Ph. D. Thesis. University of British Columbia, 1978.

[132] A. Jardy and D. Ablitzer. Proc. of Symp. Mathematical Modelling of Materials Processing Operations. Palm Springs, Cal. Nov. 29 - Dec. 2, 1987. Ed. by J. Szekely, L.B. Hales, H. Henein, N. Jarret, K. Ta jamani and I. Samarsekera. The Metallurgical Society of AIME, 1988.. Pp. 1027-1040.

[133] A. Gagnoud, D. Delage and M. Garnier. Proc. of 4th Beer-Sheva Int. Seminar on MHD Flows and Turbulence. Feb. 27-March 2, 1984. Ben Gurion University of the Negev, Beer-Sheva, Israel. Ed. by H. Branover, P.S. Lykoudis and M. Mond. The American Institute of Aeronautics and Astronautics. 1985.. Pp. 634-651.

[134] M. Etienne. Ph. D. Thesis. University of British Columbia, 1970.

[135] J. M. Sathaye. M.A.Sc. Thesis. University of British Columba, 1983.

[136] P. G. Clites.'The Inductoslag Melting Process'. U.S. Dept. of the Interior, Bureau of Mines. Bull. 673.

[137] J. Sakane, B.Q. Li and J. W. Evans. Met. Trans. B, 19B, June 1988. Pp. 397-408.

[138] O. Haida, H. Kitaoka, Y. Habu, S. Kakihara, H. Bada and S. Shiraishi. Trans. ISIJ, vol. 24, 1984, pp. 891-898.

[139] A. Mitchell. Can. Met. Quarterly, Vol. 20, No. 1, 1981, pp. 101-112.

[140] T. Takahashi, K. Ichikawa, M. Kudou and K. Shimahara. Trans. ISIJ, Vol. 16, 1976. Pp. 283-291. 
[141] H. Fredriksson, N. El Mahallawy, M. Taha, L. Xiang and G. Wänglöv. Macrosegringar och strukturförändingar av svalningskontraktion och påtvingad konvktion vid stelning av metallegeringar. Supplement VI. Inst. für Metallernas Gjutning. Stockholm, 1986. 44 pp.

[142] K. Suzuki, Y. Shinsho, K. Murata, K. Nakanishi and M. Kodana. Trans. ISIJ, vol. 24, 1984, pp. 940-949.

[143] C. G. Hill. 'Introduction to Chemical Engineering Kinetic and Reactor Design'. John Wiley \& Sons. New York, 1977. 